\title{
Global determination of two-meson distribution amplitudes from three-body $B$ decays in the perturbative QCD approach
}

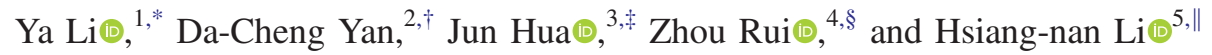 \\ ${ }^{1}$ Department of Physics, College of Sciences, Nanjing Agricultural University, \\ Nanjing, Jiangsu 210095, China \\ ${ }^{2}$ School of Mathematics and Physics, Changzhou University, Changzhou, Jiangsu 213164, China \\ ${ }^{3}$ INPAC, Key Laboratory for Particle Astrophysics and Cosmology (MOE), \\ Shanghai Key Laboratory for Particle Physics and Cosmology, School of Physics and Astronomy, \\ Shanghai Jiao-Tong University, Shanghai 200240, China \\ ${ }^{4}$ College of Sciences, North China University of Science and Technology, Tangshan, Hebei 063210, China \\ ${ }^{5}$ Institute of Physics, Academia Sinica, Taipei, Taiwan 115, Republic of China
}

(Received 19 May 2021; accepted 7 October 2021; published 19 November 2021)

\begin{abstract}
We perform a global analysis of three-body charmless hadronic decays $B \rightarrow V P_{3} \rightarrow P_{1} P_{2} P_{3}$ in the perturbative QCD (PQCD) approach, where $V$ denotes an intermediate vector resonance, and $P_{i}, i=1,2,3$ denote final-state pseudoscalar mesons. Fitting the PQCD factorization formulas at leading order in the strong coupling $\alpha_{s}$ to measured branching ratios and direct $C P$ asymmetries, we determine the Gegenbauer moments in the two-meson distribution amplitudes (DAs) for the meson pairs $P_{1} P_{2}=\pi \pi, K \pi, K K$. The fitted Gegenbauer moments are then employed to make predictions for those observables, whose data are excluded in the fit due to larger experimental uncertainties. A general consistency between our predictions and data is achieved, which hints at the validity of the PQCD formalism for the above three-body $B$-meson decays and the universality of the nonperturbative two-meson DAs. The obtained two-meson DAs can be applied to PQCD studies of other multi-body $B$-meson decays involving the same meson pairs. We also attempt to determine the dependence of the Gegenbauer moments on the meson-pair invariant mass, and demonstrate that this determination is promising, when data become more precise.
\end{abstract}

DOI: $10.1103 /$ PhysRevD.104.096014

\section{INTRODUCTION}

Since the perturbative QCD (PQCD) framework for three-body $B$-meson decays was proposed in Ref. [1], there have been extensive applications to various channels [2-15], and rich phenomenology has been explored. This formalism is based on the $k_{T}$ factorization theorem for leading-power regions of a Dalitz plot, where two finalstate mesons are roughly collimated to each other. The dominant nonperturbative dynamics responsible for the production of the meson pair, including final-state interactions between the two mesons, is absorbed into twomeson distribution amplitudes (DAs) [16-22]. It is similar

\footnotetext{
*liyakelly@163.com

†yandac@126.com

*huajun_phy@sjtu.edu.cn

\$indui1127@126.com

"hnli@phys.sinica.edu.tw
}

Published by the American Physical Society under the terms of the Creative Commons Attribution 4.0 International license. Further distribution of this work must maintain attribution to the author(s) and the published article's title, journal citation, and DOI. Funded by SCOAP. to the absorption of collinear divergences associated with a meson, which participates a high-energy QCD exclusive process, into its meson DAs. The remaining contributions, being calculable at the parton level in perturbation theory, go into hard kernels. The analysis of three-body $B$-meson decays is then simplified to that of two-body decays, where a Feynman diagram for hard kernels at leading order (LO) of the strong coupling $\alpha_{s}$ involves a single virtual gluon exchange. The same idea has recently been extended to four-body charmless hadronic $B$-meson decays [23]: they are assumed to proceed dominantly with two intermediate resonances, which then strongly decay into two light meson pairs. Various asymmetries in final-state angular distributions from the $B_{(s)} \rightarrow(K \pi)(K \pi)$ decays were predicted based on the universality of the two-meson DAs for the $K \pi$ pair.

A two-meson DA, being the time-like version of a generalized parton distribution function, depends on the parton momentum fraction $x$, the meson momentum fraction $\zeta$, which describes the relative motion between the two mesons in the pair, and the meson-pair invariant mass squared $\omega^{2}$. For the $x$ dependence, one can decompose a two-meson DA into the eigenfunctions of its 
evolution equation [24-27], i.e., the Gegenbauer polynomials $C_{n}^{3 / 2}(2 x-1)$, based on the conformal symmetry. This expansion follows that for a hadron DA exactly. As to the expansion in $\zeta$, one employs the partial waves for the produced meson pair, i.e., the Legendre polynomials $P_{l}(2 \zeta-1)$, noticing the relation $2 \zeta-1=\cos \theta$, with $\theta$ being the polar angle of a meson in the center-of-mass frame of the meson pair [28]. The expansion of a twomeson DA in terms of the two sets of orthogonal polynomials then reads [22]

$$
\begin{aligned}
\Phi\left(x, \zeta, \omega^{2}\right) & =\frac{6}{2 \sqrt{2 N_{c}}} x(1-x) \\
& \times \sum_{n=0}^{\infty} \sum_{l=0}^{n+1} B_{n l}\left(\omega^{2}\right) C_{n}^{3 / 2}(2 x-1) P_{l}(2 \zeta-1),
\end{aligned}
$$

where $B_{n l}\left(\omega^{2}\right)$ are the $\omega^{2}$-dependent coefficients, $N_{c}=3$ is the number of colors, and $l=0,1,2, \ldots$ denote the $S$-wave, $P$-wave, $D$-wave, $\ldots$ components, respectively.

The time-like form factor $B_{0 l}\left(\omega^{2}\right)$, which normalizes each of the partial-wave components, contains both resonant and nonresonant contributions. Some form factors, such as the time-like pion form factor that receives contributions from the series of $\rho$ resonances, have been constrained stringently by experimental data [29]. The other coefficients $B_{n l}\left(\omega^{2}\right)$, referred to as the Gegenbauer moments, are still quite uncertain due to a lack of systematic nonperturbative studies. Note that these Gegenbauer moments differ from those in the DA for a specific resonance which strongly decays into the meson pair, because, as stated above, a two-meson DA collects contributions from a series of resonances as well as nonresonant contributions. Moreover, they are $\omega^{2}$ dependent, a feature dramatically distinct from the Gegenbauer moments for a meson DA. It has been observed [30] that the Gegenbauer moments of a $P$-wave dipion DA differ from those of the $\rho(770)$ meson DA. Therefore, it is essential to determine the Gegenbauer moments for two-meson DAs in order to improve the precision of theoretical predictions for multibody $B$-meson decays in factorization frameworks.

We perform a global fit of the Gegenbauer moments in two-meson DAs to measured branching ratios and direct $C P$ asymmetries in three-body charmless hadronic $B$-meson decays $B \rightarrow V P_{3} \rightarrow P_{1} P_{2} P_{3}$ in the PQCD approach, where $V$ stands for an intermediate vector resonance, and $P_{i}, i=1,2,3$ stand for final-state pseudoscalar mesons. As the first attempt at a global determination of two-meson DAs, we focus on the $P$-wave components, and employ the LO PQCD factorization formulas for decay amplitudes. We establish a Gegenbauer-moment-independent database, by means of which each decay amplitude is expressed as a combination of the relevant Gegenbauer moments in the two-meson DAs. The Gegenbauer moments in the DAs for the mesons
$P_{3}=\pi, K$ are input from the global analysis of twobody $B$ meson decays in Ref. [31]. The leading-twist (twist-2) and next-to-leading-twist (twist-3) DAs for the pairs $P_{1} P_{2}=\pi \pi, K \pi$, and $K K$ with the intermediate vector mesons $V=\rho, K^{*}$, and $\phi$, respectively, are then fixed in the global fit. Because the current data for three-body $B$-meson decays are not yet precise enough to determine the $\omega^{2}$ dependence of the Gegenbauer moments, we first treat them as constant parameters defined at the initial scale $1 \mathrm{GeV}$. One or two Gegenbauer moments for each of the above two-meson DAs are obtained with satisfactory fit quality, depending on the abundance of available data. It is noticed that the results and the precision of the extracted two-meson DAs depend on the number of the Gegenbauer moments considered in the fit: when more Gegenbauer moments are introduced into the $K \pi$ DAs, the quality of the fit is improved at the cost of amplified uncertainties for fit outcomes.

The determined Gegenbauer moments are then employed to make predictions for those observables, whose data are excluded in the fit due to larger experimental errors. A general consistency between our predictions and data for various modes is achieved, except those which suffer significant subleading corrections according to previous PQCD studies, such as the $B^{0} \rightarrow \pi^{0}\left(\rho^{0} \rightarrow\right) \pi \pi$ decay [32,33]. The consistency hints at the validity of the PQCD formalism for three-body hadronic $B$-meson decays and the universality of the nonperturbative two-meson DAs. The $\pi \pi, K \pi$, and $K K$ twist- 2 and twist-3 DAs presented in this work are ready for applications to PQCD investigations of other multibody $B$-meson decays involving the same meson pairs. Our formalism can be straightforwardly extended to global fits for other twomeson DAs of various partial waves. It can also be generalized to include higher-order and/or higher-power corrections to PQCD factorization formulas [34], when they are available, so that more accurate two-meson DAs are attainable in a systematic way.

As a more ambitious attempt, we explore the dependence of the Gegenbauer moments in the dipion DAs on the pionpair invariant mass squared $\omega^{2}$. It is unlikely to determine the exact $\omega^{2}$ dependence from current data, so we simply parametrize the Gegenbauer moments up to the first power in $\omega^{2}$, following their series expansion derived in Ref. [22]. The global fit shows that at least the linear term in one of the twist-3 dipion DAs can be constrained effectively. This indicates that the determination of the $\omega^{2}$-dependent Gegenbauer moments in two-meson DAs is promising, when data become more precise in the future.

The rest of the paper is organized as follows. The kinematic variables for three-body hadronic $B$-meson decays are defined in Sec. II, where the dependence on final-state meson masses is included to describe the phase space accurately. The parton kinematics and hard kernels are then refined, such that $S U(3)$-symmetry-breaking 
effects in the decays can be evaluated more precisely. The considered two-meson $P$-wave DAs are parametrized, whose normalization form factors are assumed to take the relativistic Breit-Wigner (RBW) model [35] or the Gounaris-Sakurai (GS) model [36]. We explain how to perform the global fit, present and discuss the numerical results, and try to extract the $\omega^{2}$ dependence of the Gegenbauer moments in Sec. III, which is followed by our conclusion. We collect the PQCD factorization formulas for the decay amplitudes with numerous refined hard kernels in the Appendix.

\section{FRAMEWORK}

\section{A. Kinematics}

Consider the charmless $B$-meson decay into three pseudoscalar mesons via a vector intermediate resonance, $B\left(p_{B}\right) \rightarrow V(p) P_{3}\left(p_{3}\right) \rightarrow P_{1}\left(p_{1}\right) P_{2}\left(p_{2}\right) P_{3}\left(p_{3}\right)$, with the meson momenta $p_{B}=p+p_{3}$ and $p=p_{1}+p_{2}$. We work in the $B$-meson rest frame and parametrize the relevant momenta in the light-cone coordinates as

$$
\begin{aligned}
p_{B} & =\frac{m_{B}}{\sqrt{2}}\left(1,1, \mathbf{0}_{\mathrm{T}}\right), & k_{B} & =\left(0, x_{B} \frac{m_{B}}{\sqrt{2}}, \mathbf{k}_{B \mathrm{~T}}\right), \\
p & =\frac{m_{B}}{\sqrt{2}}\left(f_{+}, f_{-}, \mathbf{0}_{\mathrm{T}}\right), & k & =\left(z f_{+} \frac{m_{B}}{\sqrt{2}}, 0, \mathbf{k}_{\mathrm{T}}\right), \\
p_{3} & =\frac{m_{B}}{\sqrt{2}}\left(g_{-}, g_{+}, \mathbf{0}_{\mathrm{T}}\right), & k_{3} & =\left(0, x_{3} g_{+} \frac{m_{B}}{\sqrt{2}}, \mathbf{k}_{3 \mathrm{~T}}\right),
\end{aligned}
$$

where $m_{B}$ is the $B$-meson mass, and $k_{B}, k$, and $k_{3}$ are the valence quark momenta in the $B$ meson, the meson pair, and the bachelor meson with the parton momentum fraction (transverse momenta) $x_{B}, z$, and $x_{3}\left(k_{B \mathrm{~T}} k_{\mathrm{T}}\right.$, and $\left.k_{3 \mathrm{~T}}\right)$, respectively. That is, we have chosen the frame such that the meson pair and the bachelor meson move in the directions $n=\left(1,0,0_{\mathrm{T}}\right)$ and $v=\left(0,1,0_{\mathrm{T}}\right)$, respectively. Since the parton momentum $k\left(k_{3}\right)$ is aligned with the meson pair (bachelor meson), its small minus (plus) component has been neglected. We have also dropped the plus component $k_{B}^{+}$, because it does not appear in the hard kernels for dominant factorizable contributions. In the above expressions, the functions $f_{ \pm}$and $g_{ \pm}$are written as

$$
\begin{aligned}
& f_{ \pm}=\frac{1}{2}\left(1+\eta-r_{3} \pm \sqrt{(1-\eta)^{2}-2 r_{3}(1+\eta)+r_{3}^{2}}\right), \\
& g_{ \pm}=\frac{1}{2}\left(1-\eta+r_{3} \pm \sqrt{(1-\eta)^{2}-2 r_{3}(1+\eta)+r_{3}^{2}}\right),
\end{aligned}
$$

with the ratio $r_{3}=m_{P_{3}}^{2} / m_{B_{(s)}}^{2}$ and $\eta=\omega^{2} / m_{B_{(s)}}^{2}$, with $m_{P_{3}}$ being the bachelor meson mass and $\omega^{2}=p^{2}$ being the invariant mass squared of the meson pair. For a $P$-wave meson pair, we introduce the longitudinal polarization vector

$$
\epsilon=\frac{1}{\sqrt{2 \eta}}\left(f_{+},-f_{-}, \mathbf{0}_{\mathrm{T}}\right)
$$

We derive the meson momenta $p_{1}$ and $p_{2}$,

$$
\begin{aligned}
& p_{1}=\left(\left(\zeta+\frac{r_{1}-r_{2}}{2 \eta}\right) f_{+} \frac{m_{B}}{\sqrt{2}},\left(1-\zeta+\frac{r_{1}-r_{2}}{2 \eta}\right) f_{-} \frac{m_{B}}{\sqrt{2}}, \mathbf{p}_{\mathrm{T}}\right), \\
& p_{2}=\left(\left(1-\zeta-\frac{r_{1}-r_{2}}{2 \eta}\right) f_{+} \frac{m_{B}}{\sqrt{2}},\left(\zeta-\frac{r_{1}-r_{2}}{2 \eta}\right) f_{-} \frac{m_{B}}{\sqrt{2}},-\mathbf{p}_{\mathrm{T}}\right), \\
& p_{\mathrm{T}}^{2}=\zeta(1-\zeta) \omega^{2}+\frac{\left(m_{P_{1}}^{2}-m_{P_{2}}^{2}\right)^{2}}{4 \omega^{2}}-\frac{m_{P_{1}}^{2}+m_{P_{2}}^{2}}{2}
\end{aligned}
$$

from the relation $p=p_{1}+p_{2}$ and the on-shell conditions $p_{i}^{2}=m_{P_{i}}^{2}, i=1,2$, with the mass ratios $r_{1,2}=m_{P_{1}, P_{2}}^{2} / m_{B}^{2}$. The variable $\zeta+\left(r_{1}-r_{2}\right) /(2 \eta)=p_{1}^{+} / p^{+}$bears the meaning of the meson momentum fraction up to corrections from the final-state meson masses. Alternatively, one can define the polar angle $\theta$ of the meson $P_{1}$ in the $P_{1} P_{2}$ pair rest frame. The transformation between the $B$-meson rest frame and the meson pair rest frame leads to the relation between the meson momentum fraction $\zeta$ and the polar angle $\theta$,

$$
2 \zeta-1=\sqrt{1-2 \frac{r_{1}+r_{2}}{\eta}+\frac{\left(r_{1}-r_{2}\right)^{2}}{\eta^{2}}} \cos \theta,
$$

with the bounds

$$
\zeta_{\max , \min }=\frac{1}{2}\left[1 \pm \sqrt{1-2 \frac{r_{1}+r_{2}}{\eta}+\frac{\left(r_{1}-r_{2}\right)^{2}}{\eta^{2}}}\right] .
$$

We emphasize that the parametrization with the exact dependence on the final-state meson masses in Eq. (5) is crucial for establishing Eq. (6), such that the Legendre polynomials in Eq. (1) correspond to the partial waves of the meson pair exactly.

The branching ratio for a three-body $B$-meson decay is given by [37]

$$
\begin{aligned}
\int d \mathcal{B}= & \frac{\tau_{B} m_{B}}{256 \pi^{3}} \int_{\left(\sqrt{r_{1}}+\sqrt{r_{2}}\right)^{2}}^{1} d \eta \sqrt{(1-\eta)^{2}-2 r_{3}(1+\eta)+r_{3}^{2}} \\
& \times \int_{\zeta_{\min }}^{\zeta_{\max }} d \zeta|\mathcal{A}|^{2}
\end{aligned}
$$

with the $B$-meson lifetime $\tau_{B}$. The direct $C P$ asymmetry $A_{C P}$ is then defined as

$$
A_{C P}=\frac{\mathcal{B}(\bar{B} \rightarrow \bar{f})-\mathcal{B}(B \rightarrow f)}{\mathcal{B}(\bar{B} \rightarrow \bar{f})+\mathcal{B}(B \rightarrow f)} .
$$

The decay amplitude $\mathcal{A}$, according to the factorization theorem stated in the Introduction, is expressed as

$$
\mathcal{A}=\Phi_{B} \otimes H \otimes \Phi_{P_{1} P_{2}} \otimes \Phi_{P_{3}},
$$




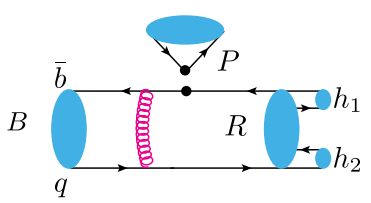

(a)

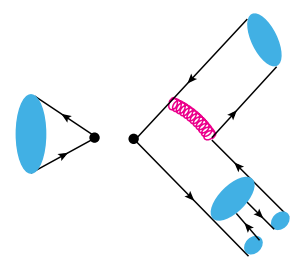

(e)

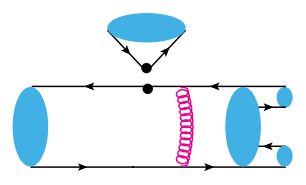

(b)

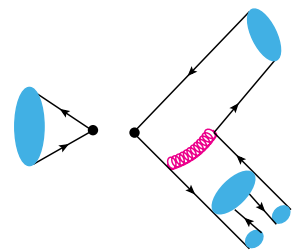

(f)

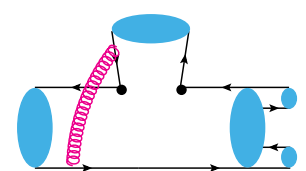

(c)

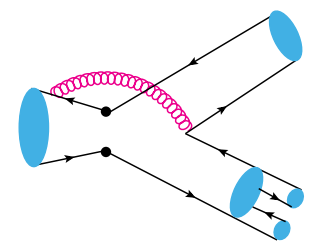

(g)

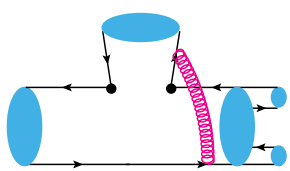

(d)

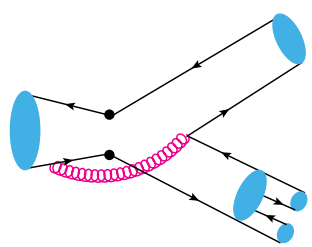

(h)

FIG. 1. LO diagrams for the three-body decays $B \rightarrow V P_{3} \rightarrow P_{1} P_{2} P_{3}$ with the light quarks $q=u, d$, $s$, where the symbol black dots represents the weak vertex.

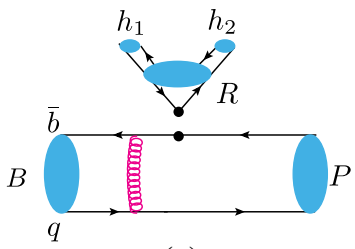

(a)

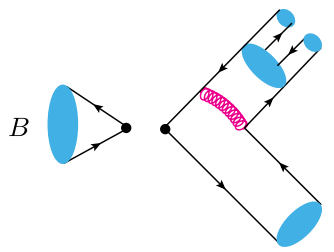

(e)

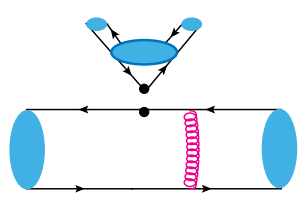

(b)

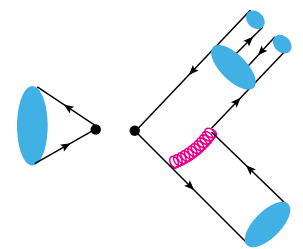

(f)

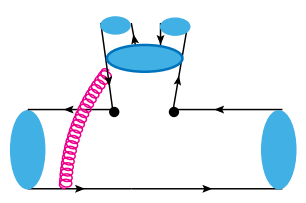

(c)

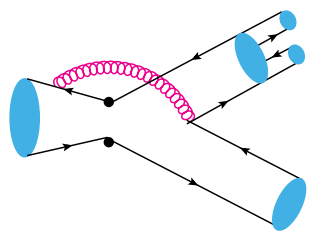

(g)

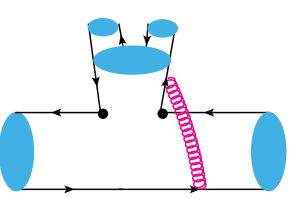

(d)

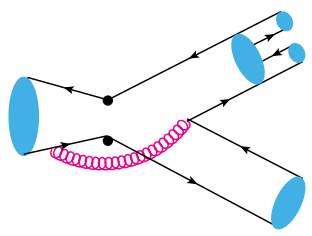

(h)

FIG. 2. More LO diagrams for the three-body decays $B \rightarrow V P_{3} \rightarrow P_{1} P_{2} P_{3}$.

where $\Phi_{B}\left(\Phi_{P_{3}}\right)$ is the $B$ (bachelor) meson DA, and the two-meson DA $\Phi_{P_{1} P_{2}}$ absorbs the nonperturbative dynamics in the production of the meson pair $P_{1} P_{2}$. The symbol $\otimes$ denotes the convolution of the above factors in parton momenta. The LO diagrams for the hard kernel $H$ are displayed in Figs. 1 and 2, where Figs. 1(a)-1(d) [Figs. 2(a)-2(d)] are associated with the $B \rightarrow P_{1} P_{2}$ $\left(B \rightarrow P_{3}\right)$ transition, and Figs. 1(e)-1(h) and 2(e)-2(h) are associated with the annihilation contributions.

\section{B. Distribution amplitudes}

The light-cone hadronic matrix element for a $B$ meson is parametrized as [38-42]

$$
\begin{aligned}
& \int d^{4} z e^{i \mathbf{k}_{1} \cdot z}\left\langle 0\left|q_{\beta}(z) \bar{b}_{\alpha}(0)\right| B\left(p_{B}\right)\right\rangle \\
& =\frac{i}{\sqrt{2 N_{c}}}\left\{\left(\not \phi_{B}+m_{B}\right) \gamma_{5}\left[\phi_{B}\left(\mathbf{k}_{\mathbf{1}}\right)-\frac{\not h-\not p}{\sqrt{2}} \bar{\phi}_{B}\left(\mathbf{k}_{\mathbf{1}}\right)\right]\right\}_{\beta \alpha},
\end{aligned}
$$

where $q$ represents a $u, d$, or $s$ quark. The two wave functions $\phi_{B}$ and $\bar{\phi}_{B}$ in the above decomposition, related to $\phi_{B}^{+}$and $\phi_{B}^{-}$ defined in the literature [43] via $\phi_{B}=\left(\phi_{B}^{+}+\phi_{B}^{-}\right) / 2$ and $\bar{\phi}_{B}=\left(\phi_{B}^{+}-\phi_{B}^{-}\right) / 2$, obey the normalization conditions

$\int \frac{d^{4} \mathbf{k}_{\mathbf{1}}}{(2 \pi)^{4}} \boldsymbol{\phi}_{B}\left(\mathbf{k}_{\mathbf{1}}\right)=\frac{f_{B}}{2 \sqrt{2 N_{c}}}, \quad \int \frac{d^{4} \mathbf{k}_{\mathbf{1}}}{(2 \pi)^{4}} \bar{\phi}_{B}\left(\mathbf{k}_{\mathbf{1}}\right)=0$.

It has been shown that the contribution from $\bar{\phi}_{B}$ is of nextto-leading power and numerically suppressed $[39,40,44]$, compared to the leading-power contribution from $\phi_{B}$. Taking the PQCD evaluation of the $B \rightarrow \pi$ transition form factor $F_{0}^{B \rightarrow \pi}$ in Ref. [44] as an example, we find that the $\bar{\phi}_{B}$ contribution to $F_{0}^{B \rightarrow \pi}$ is about $20 \%$ of the $\phi_{B}$ one. The higher-twist $B$-meson DAs have been systematically investigated in the heavy quark effective theory [45], which are decomposed according to definite twist and conformal spin assignments up to twist 6. In principle, all of the next-to-leading-power sources should be included for a consistent and complete analysis, which, however, goes beyond the scope of the present 
formalism. Therefore, we focus only on the leading-power component

$$
\Phi_{B}=\frac{i}{\sqrt{2 N_{c}}}\left(\not \phi_{B}+m_{B}\right) \gamma_{5} \phi_{B}(x, b),
$$

with the impact parameter $b$ being conjugate to the parton transverse momentum $k_{B \mathrm{~T}}$. The $B$-meson DA $\phi_{B}(x, b)$ is chosen as the model form widely adopted in the PQCD approach [38-42,46],

$\phi_{B}(x, b)=N_{B} x^{2}(1-x)^{2} \exp \left[-\frac{m_{B}^{2} x^{2}}{2 \omega_{B}^{2}}-\frac{1}{2}\left(\omega_{B} b\right)^{2}\right]$,

where the constant $N_{B}$ is related to the $B$-meson decay constant $f_{B}$ through the normalization condition $\int_{0}^{1} d x \phi_{B}(x, b=0)=f_{B} /\left(2 \sqrt{2 N_{c}}\right)$. The shape parameter takes the values $\omega_{B}=0.40 \mathrm{GeV}$ for $B^{+}, B^{0}$ mesons and $\omega_{B_{s}}=0.48 \mathrm{GeV}[31,38,47,48]$ for a $B_{s}^{0}$ meson with $10 \%$ variation in the numerical study below.

The light-cone matrix elements for a pseudoscalar meson are decomposed, up to twist 3 , into $[39,40]$

$$
\begin{aligned}
\Phi_{P} \equiv & \frac{i}{\sqrt{2 N_{C}}} \gamma_{5}\left[\not \not_{3} \phi_{P}^{A}\left(x_{3}\right)+m_{03} \phi_{P}^{P}\left(x_{3}\right)\right. \\
& \left.+m_{03}(\not h \not p-1) \phi_{P}^{T}\left(x_{3}\right)\right],
\end{aligned}
$$

with $P=\pi, K$ and the chiral scale $m_{03}$. The pion and kaon DAs have been determined at the scale $1 \mathrm{GeV}$ in a recent global analysis [31] based on LO PQCD factorization formulas, which is at the same level of accuracy as this work. The results are quoted as

$$
\begin{aligned}
\phi_{\pi}^{A}(x)= & \frac{3 f_{\pi}}{\sqrt{6}} x(1-x)\left[1+a_{2}^{\pi} C_{2}^{3 / 2}(2 x-1)\right. \\
& \left.+a_{4}^{\pi} C_{4}^{3 / 2}(2 x-1)\right], \\
\phi_{\pi}^{P}(x)= & \frac{f_{\pi}}{2 \sqrt{6}}\left[1+a_{2 P}^{\pi} C_{2}^{1 / 2}(2 x-1)\right], \\
\phi_{\pi}^{T}(x)= & \frac{f_{\pi}}{2 \sqrt{6}}(1-2 x)\left[1+a_{2 T}^{\pi}\left(10 x^{2}-10 x+1\right)\right], \\
\phi_{K}^{A}(x)= & \frac{3 f_{K}}{\sqrt{6}} x(1-x)\left[1+a_{1}^{K} C_{1}^{3 / 2}(2 x-1)\right. \\
& \left.+a_{2}^{K} C_{2}^{3 / 2}(2 x-1)+a_{4}^{K} C_{4}^{3 / 2}(2 x-1)\right], \\
\phi_{K}^{P}(x)= & \frac{f_{K}}{2 \sqrt{6}}\left[1+a_{2 P}^{K} C_{2}^{1 / 2}(2 x-1)\right], \\
\phi_{K}^{T}(x)= & -\frac{f_{K}}{2 \sqrt{6}}\left[C_{1}^{1 / 2}(x)+a_{2 T}^{K} C_{3}^{1 / 2}(x)\right],
\end{aligned}
$$

where the Gegenbauer polynomials are defined as

$$
\begin{aligned}
& C_{1}^{3 / 2}(t)=3 t, \quad C_{2}^{3 / 2}(t)=\frac{3}{2}\left(5 t^{2}-1\right), \\
& C_{4}^{3 / 2}(t)=\frac{15}{8}\left(1-14 t^{2}+21 t^{4}\right),
\end{aligned}
$$

and the Gegenbauer moments in Eq. (16) are summarized as follows:

$$
\begin{aligned}
a_{2}^{\pi}=0.64 \pm 0.08, \quad a_{4}^{\pi}=-0.41 \pm 0.10 \\
a_{2 P}^{\pi}=1.08 \pm 0.15, \quad a_{2 T}^{\pi}=-0.48 \pm 0.33 \\
a_{1}^{K}=0.33 \pm 0.08, \quad a_{2}^{K}=0.28 \pm 0.10 \\
a_{4}^{K}=-0.40 \pm 0.07, \quad a_{2 P}^{K}=0.24, \quad a_{2 T}^{K}=0.35
\end{aligned}
$$

Note that the twist-3 DAs $\phi_{K}^{P}$ and $\phi_{K}^{T}$, which were not obtained in Ref. [31], come from sum-rule calculations [49].

As stated before, we focus on the $P$-wave components in Eq. (1) proportional to $P_{l=1}(2 \zeta-1)=2 \zeta-1$. The corresponding light-cone matrix element for a longitudinal meson pair is decomposed, up to twist 3 , into [30]

$$
\begin{aligned}
\Phi_{P_{1} P_{2}}\left(x, \zeta, \omega^{2}\right)= & \frac{1}{\sqrt{2 N_{c}}}\left[\omega \phi \phi_{P_{1} P_{2}}^{0}\left(x, \omega^{2}\right)+\omega \phi_{P_{1} P_{2}}^{s}\left(x, \omega^{2}\right)\right. \\
& \left.+\frac{\not p_{1} \not \not_{2}-\not \not_{2} \not \not 1}{\omega(2 \zeta-1)} \phi_{P_{1} P_{2}}^{t}\left(x, \omega^{2}\right)\right](2 \zeta-1),
\end{aligned}
$$

where the two-meson DAs for the $\pi \pi, K K$, and $K \pi$ pairs are parametrized as

$$
\begin{aligned}
\phi_{\pi \pi}^{0}\left(x, \omega^{2}\right)= & \frac{3 F_{\pi \pi}^{\|}\left(\omega^{2}\right)}{\sqrt{2 N_{c}}} x(1-x)\left[1+a_{2 \rho}^{0} C_{2}^{3 / 2}(2 x-1)\right], \\
\phi_{\pi \pi}^{s}\left(x, \omega^{2}\right)= & \frac{3 F_{\pi \pi}^{\perp}\left(\omega^{2}\right)}{2 \sqrt{2 N_{c}}}(1-2 x)\left[1+a_{2 \rho}^{s}\left(10 x^{2}-10 x+1\right)\right], \\
\phi_{\pi \pi}^{t}\left(x, \omega^{2}\right)= & \frac{3 F_{\pi \pi}^{\perp}\left(\omega^{2}\right)}{2 \sqrt{2 N_{c}}}(1-2 x)^{2}\left[1+a_{2 \rho}^{t} C_{2}^{3 / 2}(2 x-1)\right], \\
\phi_{K \pi}^{0}\left(x, \omega^{2}\right)= & \frac{3 F_{K \pi}^{\|}\left(\omega^{2}\right)}{\sqrt{2 N_{c}}} x(1-x)\left[1+a_{1 K^{*}}^{0} C_{1}^{3 / 2}(2 x-1)\right. \\
& \left.+a_{2 K^{*}}^{0} C_{2}^{3 / 2}(2 x-1)+a_{4 K^{*}}^{0} C_{4}^{3 / 2}(2 x-1)\right], \\
\phi_{K \pi}^{s}\left(x, \omega^{2}\right)= & \frac{3 F_{K \pi}^{\perp}\left(\omega^{2}\right)}{2 \sqrt{2 N_{c}}}(1-2 x), \\
\phi_{K \pi}^{t}\left(x, \omega^{2}\right)= & \frac{3 F_{K \pi}^{\perp}\left(\omega^{2}\right)}{2 \sqrt{2 N_{c}}}(1-2 x)^{2}, \\
\phi_{K K}^{0}\left(x, \omega^{2}\right)= & \frac{3 F_{K K}^{\|}\left(\omega^{2}\right)}{\sqrt{2 N_{c}}} x(1-x)\left[1+a_{2 \phi}^{0} C_{2}^{3 / 2}(2 x-1)\right], \\
\phi_{K K}^{s}\left(x, \omega^{2}\right)= & \frac{3 F_{K K}^{\perp}\left(\omega^{2}\right)}{2 \sqrt{2 N_{c}}}(1-2 x), \\
\phi_{K K}^{t}\left(x, \omega^{2}\right)= & \frac{3 F_{K K}^{\perp}\left(\omega^{2}\right)}{2 \sqrt{2 N_{c}}}(1-2 x)^{2} .
\end{aligned}
$$

The Gegenbauer moments $a_{2 \rho}^{0, s, t}, a_{1 K^{*}, 2 K^{*}, 4 K^{*}}^{0}$, and $a_{2 \phi}^{0}$ will be determined in a global analysis in the next section. Since 
the current data are not yet precise enough to fix the Gegenbauer moments in the twist-3 DAs $\phi_{K \pi}^{s, t}$ and $\phi_{K K}^{s, t}$, they have been set to the asymptotic forms.

The elastic rescattering effects in a final-state meson pair can be absorbed into the time-like form factors $F^{\|, \perp}\left(\omega^{2}\right)$, namely, the leading Gegenbauer moment $B_{00}\left(\omega^{2}\right)$ in a two-meson DA according to the Watson theorem [50]. The resonant contribution from a $\rho$ meson with a broad width is usually parametrized as the GS model [36] based on the Breit-Wigner (BW) function [51] in experimental investigations of three-body hadronic $B$-meson decays, which interprets observed structures beyond the $\rho$ (770) resonance in terms of heavier isovector vector mesons. Taking the $\rho$ - $\omega$ interference and excitedstate contributions into account, we have the form factor $[10,29,30]$

$$
F_{\pi \pi}^{\|}\left(\omega^{2}\right)=\left[\mathrm{GS}_{\rho}\left(\omega^{2}, m_{\rho}, \Gamma_{\rho}\right) \frac{1+c_{\omega} \mathrm{BW}_{\omega}\left(\omega^{2}, m_{\omega}, \Gamma_{\omega}\right)}{1+c_{\omega}}+\Sigma c_{j} \mathrm{GS}_{j}\left(\omega^{2}, m_{j}, \Gamma_{j}\right)\right]\left(1+\Sigma c_{j}\right)^{-1},
$$

where $m_{\rho, \omega, j}\left(\Gamma_{\rho, \omega, j}\right), j=\rho^{\prime}(1450), \rho^{\prime \prime}(1700)$, and $\rho^{\prime \prime \prime}(2254)$ are the masses (decay widths) of the series of resonances, and $c_{\omega, j}$ are the weights associated with the corresponding resonances. The function $\operatorname{GS}_{\rho}\left(s, m_{\rho}, \Gamma_{\rho}\right)$ is given by

$$
\mathrm{GS}_{\rho}\left(\omega^{2}, m_{\rho}, \Gamma_{\rho}\right)=\frac{m_{\rho}^{2}\left[1+d\left(m_{\rho}\right) \Gamma_{\rho} / m_{\rho}\right]}{m_{\rho}^{2}-\omega^{2}+f\left(\omega^{2}, m_{\rho}, \Gamma_{\rho}\right)-i m_{\rho} \Gamma\left(\omega^{2}, m_{\rho}, \Gamma_{\rho}\right)},
$$

with the factors

$$
\begin{aligned}
d\left(m_{\rho}\right) & =\frac{3}{\pi} \frac{m_{\pi}^{2}}{k^{2}\left(m_{\rho}^{2}\right)} \ln \left(\frac{m_{\rho}+2 k\left(m_{\rho}^{2}\right)}{2 m_{\pi}}\right)+\frac{m_{\rho}}{2 \pi k\left(m_{\rho}^{2}\right)}-\frac{m_{\pi}^{2} m_{\rho}}{\pi k^{3}\left(m_{\rho}^{2}\right)}, \\
f\left(\omega^{2}, m_{\rho}, \Gamma_{\rho}\right) & =\frac{\Gamma_{\rho} m_{\rho}^{2}}{k^{3}\left(m_{\rho}^{2}\right)}\left\{k^{2}\left(\omega^{2}\right)\left[h\left(\omega^{2}\right)-h\left(m_{\rho}^{2}\right)\right]+\left(m_{\rho}^{2}-\omega^{2}\right) k^{2}\left(m_{\rho}^{2}\right) h^{\prime}\left(m_{\rho}^{2}\right)\right\} \\
\Gamma\left(\omega^{2}, m_{\rho}, \Gamma_{\rho}\right) & =\Gamma_{\rho} \frac{\omega^{2}}{m_{\rho}^{2}}\left[\frac{\beta_{\pi}\left(\omega^{2}\right)}{\beta_{\pi}\left(m_{\rho}^{2}\right)}\right]^{3}
\end{aligned}
$$

where the functions $k\left(\omega^{2}\right), h\left(\omega^{2}\right)$, and $\beta_{\pi}\left(\omega^{2}\right)$ are expressed as

$$
\begin{aligned}
k\left(\omega^{2}\right) & =\frac{1}{2} \sqrt{\omega^{2}} \beta_{\pi}\left(\omega^{2}\right), \\
h\left(\omega^{2}\right) & =\frac{2}{\pi} \frac{k\left(\omega^{2}\right)}{\sqrt{\omega^{2}}} \ln \left(\frac{\sqrt{\omega^{2}}+2 k\left(\omega^{2}\right)}{2 m_{\pi}}\right), \\
\beta_{\pi}\left(\omega^{2}\right) & =\sqrt{1-\frac{4 m_{\pi}^{2}}{\omega^{2}}}
\end{aligned}
$$

The function $\mathrm{BW}_{\omega}\left(\omega^{2}, m_{\omega}, \Gamma_{\omega}\right)$ for the $\omega$ resonance takes the standard BW form [51].

We apply the RBW line shape for contributions from the intermediate resonances $K^{*}$ and $\phi$ of narrow widths to the form factors $[7,8,11,35]$,

$$
F_{K \pi, K K}^{\|}\left(\omega^{2}\right)=\frac{m_{K^{*}, \phi}^{2}}{m_{K^{*}, \phi}^{2}-\omega^{2}-i m_{K^{*}, \phi} \Gamma_{K^{*}, \phi}\left(\omega^{2}\right)},
$$

with the mass-dependent widths

$$
\Gamma_{K^{*}, \phi}\left(\omega^{2}\right)=\Gamma_{K^{*}, \phi}\left(\frac{m_{K^{*}, \phi}}{\omega}\right)\left(\frac{\left|\vec{p}_{1}\right|}{\left|\vec{p}_{0}\right|}\right)^{\left(2 L_{R}+1\right)}
$$

where the masses $m_{K^{*}, \phi}$ and the widths $\Gamma_{K^{*}, \phi}$ of the $K^{*}$ and $\phi$ resonances, respectively, take the values in Ref. [37]. The magnitude of the spatial momentum of the meson $P_{1}$,

$$
\left|\vec{p}_{1}\right|=\frac{\sqrt{\lambda\left(\omega^{2}, m_{P_{1}}^{2}, m_{P_{2}}^{2}\right)}}{2 \omega}
$$

with the Källén function $\lambda(a, b, c)=a^{2}+b^{2}+c^{2}-$ $2(a b+a c+b c)$, is measured in the rest frame of the resonance, and $\left|\vec{p}_{0}\right|$ is its value at the resonance mass. The orbital angular momentum $L_{R}$ in the two-meson system is set to $L_{R}=1$ for a $P$-wave state. Due to the limited knowledge on the form factors $F^{\perp}\left(\omega^{2}\right)$, we assume the ratio $F_{i}^{\perp}\left(\omega^{2}\right) / F_{i}^{\|}\left(\omega^{2}\right) \approx\left(f_{i}^{T} / f_{i}\right)$ [30], $i=\rho, K^{*}$ and $\phi$, with $f_{i}^{T}\left(f_{i}\right)$ being the tensor (vector) decay constants of the intermediate resonances. 


\section{NUMERICAL ANALYSIS}

\section{A. Global fit}

We specify the parameters adopted in the numerical analysis below, including the masses (in units of $\mathrm{GeV}$ ) [37]

$$
\begin{aligned}
m_{B} & =5.280, \quad m_{B_{s}}=5.367, \quad m_{b}=4.8, \quad m_{K^{ \pm}}=0.494, \\
m_{K^{0}} & =0.498, \quad m_{\pi^{ \pm}}=0.140, \quad m_{\pi^{0}}=0.135,
\end{aligned}
$$

and the decay constants (in units of $\mathrm{GeV}$ ) and the $B$-meson lifetimes (in units of ps) [10,49]

$$
\begin{aligned}
f_{B} & =0.21, \quad f_{B_{s}}=0.23, \quad f_{\rho}=0.216, \quad f_{\rho}^{T}=0.184, \\
f_{\phi(1020)} & =0.215, \quad f_{\phi(1020)}^{T}=0.186, \\
f_{K^{*}} & =0.217, \quad f_{K^{*}}^{T}=0.185, \\
\tau_{B^{0}} & =1.519, \quad \tau_{B^{ \pm}}=1.638, \quad \tau_{B_{s}}=1.512 .
\end{aligned}
$$

The Wolfenstein parameters in the Cabibbo-KobayashiMaskawa (CKM) matrix take the values in Ref. [52]: $A=$ $0.836 \pm 0.015, \lambda=0.22453 \pm 0.00044, \quad \bar{\rho}=0.122_{-0.017}^{+0.018}$, and $\bar{\eta}=0.355_{-0.011}^{+0.012}$.

We stress that $\omega_{B_{(s)}}$ in the $B_{(s)}$ meson DA, as an overall parameter, cannot be determined in our global analysis, but must be treated as an input. This is why we take its value extracted from the $B_{(s)}$ meson transition form factors in lattice QCD and light-cone sum rules, which has also been verified by the global study of two-body charmless hadronic $B$-meson decays in Ref. [31]. The value of $\omega_{B_{(s)}}$, together with the corresponding pion and kaon DAs fixed in Ref. [31], are then input into the present work on the three-body $B$-meson decays for consistency. If the shape parameter $\omega_{B_{(s)}}$ is changed, the pion and kaon DAs need to be refitted accordingly before they can be employed to constrain the two-meson DAs. Fortunately, the variation of $\omega_{B_{(s)}}$ causes less than $30 \%$ uncertainties for most of the branching ratios and negligible effects on the direct $C P$ asymmetries (as seen later), and is thus not expected to make a significant impact on the determination of the twomeson DAs. Hence, we focus only on the uncertainties of the Gegenbauer moments in the two-meson DAs propagated from experimental data here.

Equation (20) suggests that the total amplitudes $\mathcal{A}$ for the $B_{(s)} \rightarrow P(\pi \pi, \pi K, K K)$ decays with $P=\pi, K$ can be expanded in terms of the Gegenbauer moments of the twomeson DAs. As a result, we decompose the squared amplitudes

$$
\begin{aligned}
\left|\mathcal{A}_{\pi \pi}\right|^{2}= & M_{0 \rho}+a_{2 \rho}^{0} M_{1 \rho}+\left(a_{2 \rho}^{0}\right)^{2} M_{2 \rho}+a_{2 \rho}^{s} M_{3 \rho} \\
& +\left(a_{2 \rho}^{s}\right)^{2} M_{4 \rho}+a_{2 \rho}^{t} M_{5 \rho}+\left(a_{2 \rho}^{t}\right)^{2} M_{6 \rho} \\
& +a_{2 \rho}^{0} a_{2 \rho}^{s} M_{7 \rho}+a_{2 \rho}^{0} a_{2 \rho}^{t} M_{8 \rho}+a_{2 \rho}^{s} a_{2 \rho}^{t} M_{9 \rho}, \\
\left|\mathcal{A}_{K \pi}\right|^{2}= & M_{0 K^{*}}+\left(a_{1 K^{*}}^{0}\right) M_{1 K^{*}}+\left(a_{1 K^{*}}^{0}\right)^{2} M_{2 K^{*}}+a_{2 K^{*}}^{0} M_{3 K^{*}} \\
& +\left(a_{2 K^{*}}^{0}\right)^{2} M_{4 K^{*}}+a_{4 K^{*}}^{0} M_{5 K^{*}}+\left(a_{4 K^{*}}^{0}\right)^{2} M_{6 K^{*}} \\
& +a_{1 K^{*}}^{0} a_{2 K^{*}}^{0} M_{7 K^{*}}+a_{1 K^{*}}^{0} a_{4 K^{*}}^{0} M_{8 K^{*}}+a_{2 K^{*}}^{0} a_{4 K^{*}}^{0} M_{9 K^{*}}, \\
\left|\mathcal{A}_{K K}\right|^{2}= & M_{0 \phi}+a_{2 \phi}^{0} M_{1 \phi}+\left(a_{2 \phi}^{0}\right)^{2} M_{2 \phi}
\end{aligned}
$$

into the linear combinations of the Gegenbauer moments $a_{2 \rho}^{0, s, t}, a_{1 K^{*}, 2 K^{*}, 4 K^{*}}^{0}$, and $a_{2 \phi}^{0}$ and their products. We then compute the coefficients $M$, which involve only the Gegenbauer polynomials, to establish the database for the global fit.

Similar to the proposal in Ref. [31], we determine the Gegenbauer moments of the two-meson DAs by fitting the formulas in Eq. (30) with the Gegenbauer-momentindependent database to the measured branching ratios $\mathcal{B}$ and direct $C P$ asymmetries $\mathcal{A}_{C P}$ of the $B_{(s)} \rightarrow P(\rho \rightarrow) \pi \pi$, $B_{(s)} \rightarrow P\left(K^{*} \rightarrow\right) K \pi$, and $B_{(s)} \rightarrow P(\phi \rightarrow) K K$ decays. We adopt the standard nonlinear least- $\chi^{2}$ (lsq) method [53], in which the $\chi^{2}$ function is defined for $n$ pieces of experimental data $v_{i} \pm \delta v_{i}$ with the errors $\delta v_{i}$ and the corresponding theoretical values $v_{i}^{\text {th }}$ as

$$
\chi^{2}=\sum_{i=1}^{n}\left(\frac{v_{i}-v_{i}^{\mathrm{th}}}{\delta v_{i}}\right)^{2} .
$$

The theoretical values $v_{i}^{\text {th }}$ are the functions of the fitted Gegenbauer moments in the two-meson DAs. The lsq fit attempts to find the smallest $\chi^{2}$ by adjusting the fitted parameters that bring the theoretical values closest to the data. The data with errors are treated as of the Gaussian type automatically in the fit packages, and the errors of the fitted parameters and the theoretical values $v_{i}^{\text {th }}$ come from experimental uncertainties by error propagation.

To minimize statistical uncertainties, we should include a maximal amount of data in the fit. On the other hand, those measurements with significance lower than $3 \sigma$ do not impose stringent constraints, and need not be taken into account in principle. The data of those modes, which are affected by subleading contributions manifestly based on the previous PQCD studies [32,33], are also excluded, even though they may have higher precision. The $B^{0} \rightarrow$ $\pi^{0}\left(\rho^{0} \rightarrow\right) \pi \pi$ decay, dominated by the color-suppressed tree amplitude that is expected to receive substantial higherorder corrections [54], is a typical example.

\section{B. Results}

The Gegenbauer moments $a_{2 \rho}^{0}, a_{2 \rho}^{s}$, and $a_{2 \rho}^{t}$ for the twist- 2 and twist- $3 \pi \pi$ DAs in Table I are obtained from the 
TABLE I. Fitted Gegenbauer moments for the twist-2 and twist-3 two-meson DAs.

\begin{tabular}{lccccc}
\hline \hline & $a_{2 \rho}^{0}$ & $a_{2 \rho}^{s}$ & $a_{2 \rho}^{t}$ & $a_{2 \phi}^{0}$ & \\
\hline Fit & $0.08 \pm 0.13$ & $-0.23 \pm 0.24$ & $-0.35 \pm 0.06$ & $-0.31 \pm 0.19$ & \\
& $a_{1 K^{*}}^{0}$ (Scenario I) & $a_{2 K^{*}}^{0}$ (Scenario I) & $a_{1 K^{*}}^{0}$ (Scenario II) & $a_{2 K^{*}}^{0}$ (Scenario II) & $a_{4 K^{*}}^{0}$ (Scenario II) \\
Fit & $0.31 \pm 0.16$ & $1.19 \pm 0.10$ & $0.57 \pm 0.20$ & $1.13 \pm 0.32$ & $-0.85 \pm 0.16$ \\
\hline \hline
\end{tabular}

fit to eight pieces of $B \rightarrow P(\rho \rightarrow) \pi \pi$ data marked by "†" in Tables II and III with $\chi^{2} /$ d.o.f. $=2.6$, whose errors mainly arise from experimental uncertainties. We point out that the measured $B^{+} \rightarrow \pi^{+}\left(\rho^{0} \rightarrow\right) \pi \pi$ branching ratio, imposing a strong constraint on the Gegenbauer moment $a_{2 \rho}^{s}$, is considered in our fit, but the corresponding $B^{+} \rightarrow \pi^{+} \rho^{0}$ data were excluded in the global analysis of two-body hadronic $B$-meson decays [31]. It is seen that our Gegenbauer moments differ from the corresponding ones of the $\rho(770)$ meson DAs derived in QCD sum rules [55] as mentioned before: the $\pi \pi$ DAs contain the $\rho-\omega$ mixing effect and the contributions from higher $\rho$ resonances with finite widths via Eq. (21), so they need not be the same as the $\rho(770)$ meson DAs. Our Gegenbauer moments also differ from $a_{2 \rho}^{0}=0.25, a_{2 \rho}^{s}=0.75$, and $a_{2 \rho}^{t}=-0.60$ chosen in Ref. [30] for two reasons, at least. First, only the $B \rightarrow K(\rho \rightarrow) \pi \pi$ data were employed to constrain the $\pi \pi$ DAs in Ref. [30], while the additional $B \rightarrow \pi(\rho \rightarrow) \pi \pi$ data are included in our global analysis. Second, some $B \rightarrow$ $K(\rho \rightarrow) \pi \pi$ data have been updated in this work.

TABLE II. $C P$ averaged branching ratios $\mathcal{B}$ and direct $C P$ asymmetries $\mathcal{A}_{C P}$ of the $B_{(s)} \rightarrow K(\rho \rightarrow) \pi \pi$ decays in the PQCD approach. The experimental data for comparison are quoted from Ref. [37]. Those data marked by $\dagger$ are included in the fit. The theoretical errors are attributed to the variations of the shape parameter $\omega_{B_{(s)}}$ in the $B_{(s)}$ meson DA and the decay constant $f_{B_{(s)}}$, of the Gegenbauer moments in the two-pion DAs, and of the hard scale $t$ and the QCD scale $\Lambda_{\mathrm{QCD}}$.

\begin{tabular}{|c|c|c|c|}
\hline Modes & & Results & Data \\
\hline \multirow[t]{2}{*}{$B^{+} \rightarrow K^{+}\left(\rho^{0} \rightarrow\right) \pi \pi$} & $\mathcal{B}\left(10^{-6}\right)$ & $2.91_{-0.60-0.68-0.82}^{+0.68+0.77+1.43}$ & $3.7 \pm 0.5^{\dagger}$ \\
\hline & $\mathcal{A}_{C P}(\%)$ & $53.5_{-1.4-4.3-15.0}^{+0.4+4.5+11.9}$ & $37 \pm 10^{\dagger}$ \\
\hline \multirow[t]{2}{*}{$B^{0} \rightarrow K^{+}\left(\rho^{-} \rightarrow\right) \pi \pi$} & $\mathcal{B}\left(10^{-6}\right)$ & $8.48_{-1.95-1.48-2.51}^{+2.20+1.63+3.87}$ & $7.0 \pm 0.9^{\dagger}$ \\
\hline & $\mathcal{A}_{C P}(\%)$ & $33.0_{-1.5-4.9-12.1}^{+1.1+5.2+8.9}$ & $20 \pm 11$ \\
\hline \multirow[t]{2}{*}{$B_{s}^{0} \rightarrow K^{-}\left(\rho^{+} \rightarrow\right) \pi \pi$} & $\mathcal{B}\left(10^{-6}\right)$ & $16.41_{-5.30-0.15-1.31}^{+7.59+0.16+1.10}$ & \\
\hline & $\mathcal{A}_{C P}(\%)$ & $19.4_{-3.2-3.3-2.9}^{+3.6+3.3+3.1}$ & \\
\hline \multirow{2}{*}{$B^{+} \rightarrow K^{0}\left(\rho^{+} \rightarrow\right) \pi \pi$} & $\mathcal{B}\left(10^{-6}\right)$ & $7.86_{-1.82-1.50-2.31}^{+2.07+1.51+3.68}$ & $7.3_{-1.2}^{+1.0 \dagger}$ \\
\hline & $\mathcal{A}_{C P}(\%)$ & $13.1_{-0.5-2.5-3.6}^{+1.2+1.8+1.5}$ & $-3 \pm 15$ \\
\hline \multirow[t]{2}{*}{$B^{0} \rightarrow K^{0}\left(\rho^{0} \rightarrow\right) \pi \pi$} & $\mathcal{B}\left(10^{-6}\right)$ & $3.76_{-0.81-0.52-0.81}^{+0.95+0.57+0.92}$ & $3.4 \pm 1.1^{\dagger}$ \\
\hline & $\mathcal{A}_{C P}(\%)$ & $1.4_{-0.5-0.6-3.1}^{+0.6+0.5+2.1}$ & $-4 \pm 20$ \\
\hline \multirow[t]{2}{*}{$B_{s}^{0} \rightarrow \bar{K}^{0}\left(\rho^{0} \rightarrow\right) \pi \pi$} & $\mathcal{B}\left(10^{-6}\right)$ & $0.17_{-0.04-0.02-0.02}^{+0.04+0.01}$ & $\ldots$ \\
\hline & $\mathcal{A}_{C P}(\%)$ & $-51.0_{-0.6-10.6-13.4}^{+1.1+11.7+26.6}$ & $\cdots$ \\
\hline
\end{tabular}

A single Gegenbauer moment $a_{2 \phi}^{0}$ is introduced into the $K K$ twist- $2 \mathrm{DA}$, and the twist- 3 ones have been set to their asymptotic forms, since only two pieces of data from the $B \rightarrow K(\phi \rightarrow) K K$ decays in Table IV meet the required precision. The value of $a_{2 \phi}^{0}$, determined with $\chi^{2} /$ d.o.f. $=0.35$, is distinct from, but still consistent with, that of the $\phi$ meson DA in QCD sum rules [55] within theoretical errors. Note that our $a_{2 \phi}^{0}$ deviates from the value $-0.50 \pm 0.10$ adopted in Ref. [4], where $B_{s}$ meson decays into charmonia plus a kaon pair were investigated. The deviation is understandable, because the choice of $a_{2 \phi}^{0}$ depends on models for the uncertain charmonium DAs, as relevant data were accommodated.

The $K \pi$ DAs are determined in a fit to six pieces of $B_{(s)} \rightarrow P\left(K^{*} \rightarrow\right) K \pi$ data in Tables $V$ and VI. We first work on Scenario I, in which the two Gegenbauer moments $a_{1 K^{*}}^{0}$ and $a_{2 K^{*}}^{0}$ of the twist-2 two-meson DA are fitted with $\chi^{2} /$ d.o.f. $=1.5$, and observe that $a_{2 K^{*}}^{0}$ is slightly larger than unity, as shown in Table I. A larger moment is not favored in view of the convergence of the Gegenbauer

TABLE III. Same as Table II but for the $B_{(s)} \rightarrow \pi(\rho \rightarrow) \pi \pi$ decays.

\begin{tabular}{lccc}
\hline \hline Modes & & Results & Data \\
\hline$B^{+} \rightarrow \pi^{+}\left(\rho^{0} \rightarrow\right) \pi \pi$ & $\mathcal{B}\left(10^{-6}\right)$ & $5.98_{-1.37-1.31-0.37}^{+1.56+1.46+0.4}$ & $8.3 \pm 1.2^{\dagger}$ \\
& $\mathcal{A}_{C P}(\%)$ & $-34.9_{-0.7-4.4-9.6}^{+2.0+5.7}$ & $0.9 \pm 1.9$ \\
$B^{0} \rightarrow \pi^{+}\left(\rho^{-} \rightarrow\right) \pi \pi$ & $\mathcal{B}\left(10^{-6}\right)$ & $5.28_{-1.58-1.44-0.52}^{+2.08+1.56+0.42}$ & $23.0 \pm 2.3^{1 \dagger}$ \\
& $\mathcal{A}_{C P}(\%)$ & $-30.6_{-3.5-4.1-5.4}^{+3.4+4.1+4.5}$ & $-8 \pm 8$ \\
$B^{0} \rightarrow \pi^{-}\left(\rho^{+} \rightarrow\right) \pi \pi$ & $\mathcal{B}\left(10^{-6}\right)$ & $20.20_{-6.62-0.54-1.04}^{+8.90+0.48+1.30}$ & $23.0 \pm 2.3^{1 \dagger}$ \\
& $\mathcal{A}_{C P}(\%)$ & $9.3_{-1.6-1.7-1.9}^{+1.9+1.7+1.9}$ & $13 \pm 6$ \\
$B_{s}^{0} \rightarrow \pi^{+}\left(\rho^{-} \rightarrow\right) \pi \pi$ & $\mathcal{B}\left(10^{-6}\right)$ & $0.23_{-0.04-0.05-0.04}^{+0.04+0.03+0.03}$ & $\ldots$ \\
& $\mathcal{A}_{C P}(\%)$ & $-24.3_{-3.8-14.3-6.1}^{+2.0+4.5+8.8}$ & $\ldots$ \\
$B_{s}^{0} \rightarrow \pi^{-}\left(\rho^{+} \rightarrow\right) \pi \pi$ & $\mathcal{B}\left(10^{-6}\right)$ & $0.12_{-0.05-0.06-0.06}^{+0.01+0.01+0.0}$ & $\ldots$ \\
& $\mathcal{A}_{C P}(\%)$ & $-71.7_{-1.8-5.6-0.7}^{+2.1+12.0+4}$ & $\ldots$ \\
$B^{+} \rightarrow \pi^{0}\left(\rho^{+} \rightarrow\right) \pi \pi$ & $\mathcal{B}\left(10^{-6}\right)$ & $8.50_{-3.04-0.98-0.55}^{+4.25+1.05+0.24}$ & $10.9 \pm 1.4^{\dagger}$ \\
& $\mathcal{A}_{C P}(\%)$ & $20.4_{-4.1-4.4-6.4}^{+5.0+4.6+4.7}$ & $2 \pm 11$ \\
$B^{0} \rightarrow \pi^{0}\left(\rho^{0} \rightarrow\right) \pi \pi$ & $\mathcal{B}\left(10^{-6}\right)$ & $0.08_{-0.02-0.03-0.05}^{+0.01+0.02+0.05}$ & $2.0 \pm 0.5$ \\
& $\mathcal{A}_{C P}(\%)$ & $20.8_{-4.4-16.5-40.1}^{+6.0+17.0+11.7}$ & $27 \pm 24$ \\
$B_{s}^{0} \rightarrow \pi^{0}\left(\rho^{0} \rightarrow\right) \pi \pi$ & $\mathcal{B}\left(10^{-6}\right)$ & $0.14_{-0.03-0.01-0.04}^{+0.03+0.04+0.04}$ & $\ldots$ \\
& $\mathcal{A}_{C P}(\%)$ & $-47.9_{-3.0-6.6-7.5}^{+5.5+4.8+4.5}$ & $\ldots$ \\
\hline \hline
\end{tabular}

${ }^{1}$ Sum of two branching ratios, $\mathcal{B}(B \rightarrow f)+\mathcal{B}(B \rightarrow \bar{f})$. 
TABLE IV. Same as Table II but for the $B_{(s)} \rightarrow P(\phi \rightarrow) K K$ decays with $P=\pi, K$.

\begin{tabular}{|c|c|c|c|}
\hline Modes & & Results & Data \\
\hline \multirow[t]{2}{*}{$B^{+} \rightarrow K^{+}(\phi \rightarrow) K K$} & $\mathcal{B}\left(10^{-6}\right)$ & $8.46_{-2.70-0.45-1.95}^{+3.57+0.41+2.65}$ & $8.8_{-0.6}^{+0.7 \dagger}$ \\
\hline & $\mathcal{A}_{C P}(\%)$ & $1.4_{-0.3-1.7-0.8}^{+0.8+0.0}$ & $2.4 \pm 2.8$ \\
\hline \multirow[t]{2}{*}{$B^{0} \rightarrow K^{0}(\phi \rightarrow) K K$} & $\mathcal{B}\left(10^{-6}\right)$ & $7.82_{-2.50-0.19-1.71}^{+3.18+0.40}$ & $7.3 \pm 0.7^{\dagger}$ \\
\hline & $\mathcal{A}_{C P}(\%)$ & 0 & $1 \pm 14$ \\
\hline \multirow[t]{2}{*}{$B_{s}^{0} \rightarrow \bar{K}^{0}(\phi \rightarrow) K K$} & $\mathcal{B}\left(10^{-8}\right)$ & $3.52_{-0.64-0.02-1.27}^{+1.30+1.50+2.30}$ & \\
\hline & $\mathcal{A}_{C P}(\%)$ & 0 & $\ldots$ \\
\hline \multirow[t]{2}{*}{$B^{+} \rightarrow \pi^{+}(\phi \rightarrow) K K$} & $\mathcal{B}\left(10^{-8}\right)$ & $1.15_{-0.33-0.20-0.28}^{+0.46+0.02+0.34}$ & $3.2 \pm 1.5$ \\
\hline & $\mathcal{A}_{C P}(\%)$ & 0 & $10 \pm 50$ \\
\hline \multirow[t]{2}{*}{$B^{0} \rightarrow \pi^{0}(\phi \rightarrow) K K$} & $\mathcal{B}\left(10^{-9}\right)$ & $5.32_{-1.53-0.91-1.27}^{+2.21+0.14+1.61}$ & $<15$ \\
\hline & $\mathcal{A}_{C P}(\%)$ & 0 & $\ldots$ \\
\hline \multirow[t]{2}{*}{$B_{s}^{0} \rightarrow \pi^{0}(\phi \rightarrow) K K$} & $\mathcal{B}\left(10^{-7}\right)$ & $1.06_{-0.34-0.20-0.14}^{+0.41+0.15+0.07}$ & $\cdots$ \\
\hline & $\mathcal{A}_{C P}(\%)$ & $27.3_{-1.0-1.4-5.8}^{+1.1+3.5}$ & .. \\
\hline
\end{tabular}

expansion. Therefore, one more Gegenbauer moment $a_{4 K^{*}}^{0}$ is added in Scenario II, and a fit with $\chi^{2} /$ d.o.f. $=1.4$ is attained. The resultant $a_{2 K^{*}}^{0}$ decreases a bit but with amplified uncertainty, and $a_{4 K^{*}}^{0}$ is smaller than unity. The measured $B_{s}^{0} \rightarrow K^{ \pm}\left(K^{* \mp} \rightarrow\right) K \pi$ and $\left.B_{s}^{0} \rightarrow \stackrel{(-)^{0}}{K} \stackrel{(-) * 0}{K} \rightarrow\right) K \pi$ branching ratios cannot give an effective constraint due to their larger experimental errors, such that the uncertainties of the Gegenbauer moments increase dramatically in Scenario II. For a similar reason, the obtained Gegenbauer moments differ from those of the $K^{*}$ meson DA in QCD sum rules [55], and from $a_{1 K^{*}}^{0}=0.2$ and $a_{2 K^{*}}^{0}=0.5$ chosen in the PQCD study on the $B_{(s)} \rightarrow \psi K \pi$ decays [56]. We state that the fits based on the currently available data cannot discriminate the two scenarios effectively. As experimental precision increases, we will be able to impose more stringent constraints on those twomeson DAs.

With the fitted Gegenbauer moments in Table I, we calculate the $C P$ averaged branching ratios $\mathcal{B}$ and the direct $C P$ asymmetries $\mathcal{A}_{C P}$ in the LO PQCD formalism, and present the results in the central columns of Tables II-VI. The first theoretical uncertainty originates from the shape parameter $\omega_{B}=0.40 \mathrm{GeV}$ or $\omega_{B_{s}}=0.48 \mathrm{GeV}$ with $10 \%$ variation, and the decay constant $f_{B_{(s)}}$. The second one is from the Gegenbauer moments in the two-meson DAs. The last one is caused by the variations of the hard scale $t$ from $0.75 t$ to $1.25 t$, which characterizes the effect of next-to-leading-order QCD corrections, and of the QCD scale $\Lambda_{\mathrm{QCD}}=0.25 \pm 0.05 \mathrm{GeV}$. The errors attributed to the CKM matrix elements are tiny and can be safely ignored. Note that the data for the $B^{0} \rightarrow \pi^{+}\left(\rho^{-} \rightarrow\right) \pi \pi$

TABLE V. Same as Table II but for the $B_{(s)} \rightarrow K\left(K^{*} \rightarrow\right) K \pi$ decays.

\begin{tabular}{|c|c|c|c|c|}
\hline Modes & & Results (scenario I) & Results (scenario II) & Data \\
\hline \multirow[t]{2}{*}{$B^{+} \rightarrow K^{+}\left(\bar{K}^{* 0} \rightarrow\right) K \pi$} & $\mathcal{B}\left(10^{-6}\right)$ & $0.55_{-0.13-0.06-0.14}^{+0.14+0.04+0.20}$ & $0.56_{-0.13-0.06-0.13}^{+0.17+0.10+0.15}$ & $0.59 \pm 0.08^{\dagger}$ \\
\hline & $\mathcal{A}_{C P}(\%)$ & $46.3_{-0.3-4.7-3.9}^{+1.0+10.9+2.8}$ & $63.8_{-3.2-8.5-23.5}^{+1.1+2.0+3.4}$ & $12 \pm 10$ \\
\hline \multirow[t]{2}{*}{$B^{0} \rightarrow K^{+}\left(K^{*-} \rightarrow\right) K \pi$} & $\mathcal{B}\left(10^{-6}\right)$ & $0.27_{-0.05-0.06-0.03}^{+0.05+0.05+0.04}$ & $0.25_{-0.01-0.03-0.01}^{+0.01+0.09+0.01}$ & $<0.4^{1}$ \\
\hline & $\mathcal{A}_{C P}(\%)$ & $19.8_{-3.6-2.1-7.5}^{+0.5+2.1+13.4}$ & $20.2_{-0.0-1.6-0.0}^{+7.1+10.6+16.9}$ & $\cdots$ \\
\hline \multirow[t]{2}{*}{$B^{0} \rightarrow K^{-}\left(K^{*+} \rightarrow\right) K \pi$} & $\mathcal{B}\left(10^{-6}\right)$ & $0.09_{-0.02-0.01-0.03}^{+0.01+0.01+0.04}$ & $0.11_{-0.06-0.02-0.02}^{+0.02+0.01+0.03}$ & $<0.4^{1}$ \\
\hline & $\mathcal{A}_{C P}(\%)$ & $-5.2_{-15.5-11.4-0.0}^{+12.3+9.4+30.4}$ & $33.8_{-0.0-14.4-0.0}^{+13.4+16.4+9.4}$ & $\cdots$ \\
\hline \multirow[t]{2}{*}{$B_{s}^{0} \rightarrow K^{+}\left(K^{*-} \rightarrow\right) K \pi$} & $\mathcal{B}\left(10^{-6}\right)$ & $15.15_{-2.53-1.72-4.61}^{+2.78+1.90+7.29}$ & $9.89_{-1.66-1.90-4.16}^{+1.92+2.93+5.64}$ & $(19 \pm 5)^{1 \dagger}$ \\
\hline & $\mathcal{A}_{C P}(\%)$ & $42.1_{-5.3-3.6-6.9}^{+4.5+2.4+5.5}$ & $6.1_{-1.3-11.0-10.4}^{+0.4+8.8+7.0}$ & $\cdots$ \\
\hline \multirow[t]{2}{*}{$B_{s}^{0} \rightarrow K^{-}\left(K^{*+} \rightarrow\right) K \pi$} & $\mathcal{B}\left(10^{-6}\right)$ & $10.22_{-1.73-1.24-2.72}^{+1.97+1.27+4.51}$ & $7.72_{-1.59-1.49-2.69}^{+1.88+1.82+3.24}$ & $(19 \pm 5)^{1 \dagger}$ \\
\hline & $\mathcal{A}_{C P}(\%)$ & $-34.8_{-2.3-0.6-6.6}^{+3.0+1.5+7.5}$ & $-24.0_{-0.3-4.1-6.1}^{+1.5+6.1+11.4}$ & $\cdots$ \\
\hline \multirow[t]{2}{*}{$B^{+} \rightarrow \bar{K}^{0}\left(K^{*+} \rightarrow\right) K \pi$} & $\mathcal{B}\left(10^{-6}\right)$ & $0.31_{-0.05-0.04-0.09}^{+0.06+0.07+0.16}$ & $0.19_{-0.05-0.07-0.05}^{+0.06+0.06+0.11}$ & $\cdots$ \\
\hline & $\mathcal{A}_{C P}(\%)$ & $-13.6_{-1.0-3.5-7.9}^{+2.5+2.0+5.7}$ & $-22.7_{-0.0-18.4-7.3}^{+13.3+20.7+7.5}$ & $\cdots$ \\
\hline \multirow[t]{2}{*}{$B^{0} \rightarrow K^{0}\left(\bar{K}^{* 0} \rightarrow\right) K \pi$} & $\mathcal{B}\left(10^{-6}\right)$ & $0.44_{-0.11+0.03+0.11}^{+0.14+0.04+0.15}$ & $0.38_{-0.11-0.04-0.11}^{+0.13+0.05+0.11}$ & $<0.96^{1}$ \\
\hline & $\mathcal{A}_{C P}(\%)$ & 0 & 0 & $\ldots$ \\
\hline \multirow[t]{2}{*}{$B^{0} \rightarrow \bar{K}^{0}\left(K^{* 0} \rightarrow\right) K \pi$} & $\mathcal{B}\left(10^{-6}\right)$ & $0.44_{-0.08+0.07+0.15}^{+0.08+0.06+0.22}$ & $0.30_{-0.05-0.02-0.12}^{+0.07+0.08+0.16}$ & $<0.96^{1}$ \\
\hline & $\mathcal{A}_{C P}(\%)$ & 0 & 0 & $\cdots$ \\
\hline \multirow[t]{2}{*}{$B_{s}^{0} \rightarrow K^{0}\left(\bar{K}^{* 0} \rightarrow\right) K \pi$} & $\mathcal{B}\left(10^{-6}\right)$ & $14.06_{-2.30-1.70-4.48}^{+2.54+1.89+6.88}$ & $8.84_{-1.46-1.98-3.54}^{+1.66+2.77+5.31}$ & $(20 \pm 6)^{1 \dagger}$ \\
\hline & $\mathcal{A}_{C P}(\%)$ & 0 & 0 & $\ldots$ \\
\hline \multirow[t]{2}{*}{$B_{s}^{0} \rightarrow \bar{K}^{0}\left(K^{* 0} \rightarrow\right) K \pi$} & $\mathcal{B}\left(10^{-6}\right)$ & $10.39_{-1.78-1.17-2.86}^{+2.01+1.18+5.58}$ & $\begin{array}{c}7.92_{-1.64-1.36-2.85}^{+1.95+1.63+3.46} \\
\end{array}$ & $(20 \pm 6)^{1 \dagger}$ \\
\hline & $\mathcal{A}_{C P}(\%)$ & 0 & 0 & $\cdots$ \\
\hline
\end{tabular}


TABLE VI. Same as Table II but for the $B_{(s)} \rightarrow \pi\left(K^{*} \rightarrow\right) K \pi$ decays.

\begin{tabular}{|c|c|c|c|c|}
\hline Modes & & Results (scenario I) & Results (scenario II) & Data \\
\hline \multirow[t]{2}{*}{$B^{+} \rightarrow \pi^{+}\left(K^{* 0} \rightarrow\right) K \pi$} & $\mathcal{B}\left(10^{-6}\right)$ & $7.17_{-1.37-0.62-2.23}^{+1.56+0.64+3.46}$ & $8.19_{-1.77-0.66-1.93}^{+2.14+0.94+2.74}$ & $10.1 \pm 0.8$ \\
\hline & $\mathcal{A}_{C P}(\%)$ & $-5.4_{-0.2-0.3-0.8}^{+0.5+0.8+2.1}$ & $-4.5_{-0.6-1.4-1.2}^{+0.5+1.1+2.7}$ & $-4 \pm 9$ \\
\hline \multirow[t]{2}{*}{$B^{0} \rightarrow \pi^{-}\left(K^{*+} \rightarrow\right) K \pi$} & $\mathcal{B}\left(10^{-6}\right)$ & $7.47_{-1.41-0.71-2.06}^{+1.60+0.72+3.29}$ & $7.61_{-1.61-0.65-1.78}^{+1.83+0.92+2.40}$ & $7.5 \pm 0.4^{\dagger}$ \\
\hline & $\mathcal{A}_{C P}(\%)$ & $-52.9_{-1.8-1.0-7.0-7.0}^{+3.1+0.3}$ & $-32.3_{-0.2-8.4-6.3}^{+0.7+10.9}$ & $-27 \pm 4$ \\
\hline \multirow[t]{2}{*}{$B_{s}^{0} \rightarrow \pi^{+}\left(K^{*-} \rightarrow\right) K \pi$} & $\mathcal{B}\left(10^{-6}\right)$ & $12.13_{-3.55-1.29-0.75}^{+4.66+1.36+0.92}$ & $5.52_{-1.66-1.85-0.41}^{+2.22+2.09+0.41}$ & $2.9 \pm 1.1$ \\
\hline & $\mathcal{A}_{C P}(\%)$ & $-32.8_{-4.8-3.2-5.5}^{+4.1+2.7+4.2}$ & $-30.6_{-4.5-6.9-8.9}^{+4.1+6.5+8.2}$ & $\ldots$ \\
\hline \multirow[t]{2}{*}{$B^{+} \rightarrow \pi^{0}\left(K^{*+} \rightarrow\right) K \pi$} & $\mathcal{B}\left(10^{-6}\right)$ & $4.71_{-0.98-0.38-1.30}^{+1.18+0.39+1.92}$ & $5.62_{-1.28-0.49-1.11}^{+1.54+0.62+1.55}$ & $6.8 \pm 0.9^{\dagger}$ \\
\hline & $\mathcal{A}_{C P}(\%)$ & $-36.2_{-1.0-0.4-8.2}^{+1.6+0.1+7.4}$ & $-19.1_{-1.9-5.4-6.0}^{+2.4+6.6+4.6}$ & $-39 \pm 21$ \\
\hline \multirow[t]{2}{*}{$B^{0} \rightarrow \pi^{0}\left(K^{* 0} \rightarrow\right) K \pi$} & $\mathcal{B}\left(10^{-6}\right)$ & $2.99_{-0.55-0.33-0.89}^{+0.59+0.33+1.49}$ & $2.55_{-0.49-0.19-0.74}^{+0.57+0.36+1.06}$ & $3.3 \pm 0.6^{\dagger}$ \\
\hline & $\mathcal{A}_{C P}(\%)$ & $-11.6_{-1.2-0.2-1.0}^{+1.0+0.49+5.0}$ & $-11.8_{-1.2-1.7-0.2}^{+1.2+4.3+4.3}$ & $-15 \pm 13$ \\
\hline \multirow{2}{*}{$B_{s}^{0} \rightarrow \pi^{0}\left(\bar{K}^{* 0} \rightarrow\right) K \pi$} & $\mathcal{B}\left(10^{-6}\right)$ & $0.20_{-0.04-0.02-0.05}^{+0.03+0.01+06}$ & $0.12_{-0.04-0.03-0.03}^{+0.02+0.02+03}$ & $\ldots$ \\
\hline & $\mathcal{A}_{C P}(\%)$ & $-70.6_{-6.7-2.8-15.1}^{+6.7+13.2+23.5}$ & $-50.4_{-2.6-12.4-14.1}^{+3.1+22.7+15.1}$ & \\
\hline
\end{tabular}

and $B^{0} \rightarrow \pi^{-}\left(\rho^{+} \rightarrow\right) \pi \pi$ branching ratios in Table III represent the sum over these two modes. It is also the case for the measured $B_{s}^{0} \rightarrow K^{+}\left(K^{*-} \rightarrow\right) K \pi$ and $B^{+} \rightarrow$ $\pi^{0}\left(K^{*+} \rightarrow\right) K \pi$ branching ratios, and for the measured $B^{0} \rightarrow \pi^{0}\left(K^{* 0} \rightarrow\right) K \pi$ and $B_{s}^{0} \rightarrow \bar{K}^{0}\left(K^{* 0} \rightarrow\right) K \pi$ branching ratios in Table $\mathrm{V}$.
One can also assess the uncertainties from the Gegenbauer moments $a_{2,4}^{\pi}, a_{2 P(T)}^{\pi}$, and $a_{(1,2,4)}^{K}$ in the pion and kaon DAs. Taking the $B^{+} \rightarrow \pi^{+}\left(\rho^{0} \rightarrow\right) \pi \pi$ and $B_{s}^{0} \rightarrow$ $K^{-}\left(K^{*+} \rightarrow\right) K \pi$ branching ratios in Scenario II as examples, we obtain, given the errors in Eq. (18),

$$
\begin{aligned}
\mathcal{B}\left(B^{+} \rightarrow \pi^{+}\left(\rho^{0} \rightarrow\right) \pi \pi\right) & =\left(5.98_{-0.02}^{+0.02}\left(a_{2}^{\pi}\right)_{-0.26}^{+0.24}\left(a_{4}^{\pi}\right)_{-0.06}^{+0.07}\left(a_{2 P}^{\pi}\right)_{-0.01}^{+0.01}\left(a_{2 T}^{\pi}\right)\right) \times 10^{-6} \\
\mathcal{B}\left(B_{s}^{0} \rightarrow K^{-}\left(K^{*+} \rightarrow\right) K \pi\right) & =\left(7.72_{-0.14}^{+0.16}\left(a_{1}^{K}\right)_{-0.35}^{+0.34}\left(a_{2}^{K}\right)_{-0.14}^{+0.13}\left(a_{4}^{K}\right)\right) \times 10^{-6}
\end{aligned}
$$

It is seen that the former (latter) is more sensitive to the variation of the moment $a_{4}^{\pi}\left(a_{2}^{K}\right)$ in the twist-2 pion (kaon) DA. We remark that the total errors, derived by adding the individual ones from the moments in the pion and kaon DAs in quadrature and associated with the labels $a^{\pi}$ and $a^{K}$ below, respectively, are minor (less than 5\%) compared with other uncertainties listed in Tables III and V:

$$
\begin{aligned}
\mathcal{B}\left(B^{+} \rightarrow \pi^{+}\left(\rho^{0} \rightarrow\right) \pi \pi\right) & =\left(5.98_{-1.37}^{+1.56}\left(\omega_{B}, f_{B}\right)_{-0.26}^{+0.25}\left(a^{\pi}\right)_{-1.31}^{+1.46}\left(a_{\rho}\right)_{-0.37}^{+0.45}\left(t, \Lambda_{\mathrm{QCD}}\right)\right) \times 10^{-6}, \\
\mathcal{B}\left(B_{s}^{0} \rightarrow K^{-}\left(K^{*+} \rightarrow\right) K \pi\right) & =\left(7.72_{-1.59}^{+1.89}\left(\omega_{B}, f_{B}\right)_{-0.40}^{+0.40}\left(a^{K}\right)_{-1.49}^{+1.82}\left(a_{K^{*}}\right)_{-2.69}^{+3.24}\left(t, \Lambda_{\mathrm{QCD}}\right)\right) \times 10^{-6} .
\end{aligned}
$$

Therefore, the variation of the Gegenbauer moments in the pion and kaon DAs has little impact on the determination of the two-meson DAs.

It is found that most of the considered data in Tables II and III are well reproduced, in particular those with higher precision. Larger deviation from the data is observed in the $B^{+} \rightarrow \pi^{+}\left(\rho^{0} \rightarrow\right) \pi \pi$ and $B^{+} \rightarrow \pi^{0}\left(\rho^{+} \rightarrow\right) \pi \pi$ branching ratios. It is ascribed to the involved color-suppressed tree contributions, which receive sizable next-to-leading-order corrections. The observables removed from the fit are also predicted in the LO PQCD formalism, and compared with the data in Tables II and III. Our prediction for the $B^{0} \rightarrow$ $\pi^{0}\left(\rho^{0} \rightarrow\right) \pi \pi$ branching ratio, which suffers significant subleading corrections as stated before, is still below the data, similar to that derived in the framework for two-body decays. Most of the $\mathcal{A}_{C P}$ data for the $B_{(s)} \rightarrow P(\rho \rightarrow) \pi \pi$ decays with $P=\pi, K$ are not yet precise enough. We mention that $\mathcal{A}_{C P}$ in the $B^{+} \rightarrow \pi^{+} \rho^{0}$ mode has been predicted to be large and negative in most QCD approaches [10,57], including the present analysis on three-body decays, as shown in Table III. However, its data are as small as $0.009 \pm 0.019$ [37]. Both the theoretical and experimental errors need to be reduced greatly in order to tell whether the discrepancy really stands as a puzzle.

Both the $B \rightarrow K(\phi \rightarrow) K K$ data considered in the fit are well reproduced with a single Gegenbauer moment $a_{2 \phi}^{0}$, as indicated in Table IV. Our predictions for the branching ratios and direct $C P$ asymmetries excluded in the fit, mainly associated with $B_{s}$ meson decays, can be confronted by more precise data in the future. All of the available 
TABLE VII. $C P$ averaged branching ratios $\mathcal{B}\left(10^{-6}\right)$ corresponding to the fitted Gegenbauer moments in Eq. (34), compared with the data [37]. For simplicity, only the theoretical errors from the Gegenbauer moments are presented.

\begin{tabular}{lcc}
\hline \hline Modes & Results & Data \\
\hline$B^{+} \rightarrow K^{+}\left(\bar{K}^{* 0} \rightarrow\right) K \pi$ & $0.59 \pm 0.08$ & $3.7 \pm 0.5^{\dagger}$ \\
$B^{0} \rightarrow \pi^{-}\left(K^{*+} \rightarrow\right) K \pi$ & $7.51 \pm 0.34$ & $7.5 \pm 0.4^{\dagger}$ \\
$B^{+} \rightarrow \pi^{0}\left(K^{*+} \rightarrow\right) K \pi$ & $4.75 \pm 0.37$ & $6.8 \pm 0.9^{\dagger}$ \\
$B^{0} \rightarrow \pi^{0}\left(K^{* 0} \rightarrow\right) K \pi$ & $2.91 \pm 0.40$ & $3.3 \pm 0.6^{\dagger}$ \\
$B_{s}^{0} \rightarrow K^{+}\left(K^{*-} \rightarrow\right) K \pi+c . c$ & $25.40 \pm 1.60$ & $19 \pm 5^{\dagger}$ \\
$B_{s}^{0} \rightarrow K^{0}\left(\bar{K}^{* 0} \rightarrow\right) K \pi+c . c$ & $24.60 \pm 1.50$ & $20 \pm 6^{\dagger}$ \\
\hline \hline
\end{tabular}

$\mathcal{A}_{C P}$ data for the $B \rightarrow P(\phi \rightarrow) K K$ decays with $P=\pi, K$ have large errors. The central values of the prediction and the data for the $B^{+} \rightarrow \pi^{+}(\phi \rightarrow) K K$ branching ratio are different, but still agree with each other within uncertainties.

Generally speaking, Scenario II reproduces the considered $B_{(s)} \rightarrow P\left(K^{*} \rightarrow\right) K \pi$ data with $P=\pi, K$ slightly better than Scenario I does, as seen in Tables V and VI. The $B_{s} \rightarrow$ $P\left(K^{*} \rightarrow\right) K \pi$ branching ratios differ between the two scenarios more than the $B \rightarrow P\left(K^{*} \rightarrow\right) K \pi$ branching ratios do. This feature is understandable, because the former involve the $B_{s} \rightarrow\left(K^{*} \rightarrow\right) K \pi$ transition form factors, which are more sensitive to the variation of the Gegenbauer moments in the $K \pi$ DA. Hence, more precise $B_{s} \rightarrow$ $P\left(K^{*} \rightarrow\right) K \pi$ data are crucial for fixing the $K \pi$ DAs. The direct $C P$ asymmetries $\mathcal{A}_{C P}$ in some $B_{(s)} \rightarrow P\left(K^{*} \rightarrow\right) K \pi$ modes strongly depend on the chosen scenarios, implying that more accurate $K \pi$ DAs are necessary to unambiguously predict these observables. The central value of the predicted $B_{s}^{0} \rightarrow \pi^{+}\left(K^{*-} \rightarrow\right) K \pi$ branching ratio in Scenario II, which is already much lower than in Scenario I, remains above the data. It deserves more thorough theoretical and experimental investigations. Similarly, most of the $\mathcal{A}_{C P}$ data for the $B_{(s)} \rightarrow P\left(K^{*} \rightarrow\right) K \pi$ decays have substantial uncertainties so far, so it is not yet possible to make a meaningful comparison with our results.

A remark is in order. The twist-2 DAs $\phi_{K}^{A}$ and $\phi_{K \pi}^{0}$ in Eqs. (16) and (20), respectively, are expanded up to the fourth-order Gegenbauer polynomial without the third-order term, which exists in general. We find that the $S U(3)$ breaking effects in the considered decays can be well accounted for by the first-order term alone. That is, even when the third-order term is included in the fit, its value turns out to be small, and does not modify the fit outcomes much. Taking the eight $B_{(s)} \rightarrow P\left(K^{*} \rightarrow\right) K \pi(P=\pi, K)$ decays as examples, we perform the global fit with $a_{3 K^{*}}^{0}$ being included, and obtain the Gegenbauer moments of the $K \pi$ twist-2 DA,

$a_{1 K^{*}}^{0}=0.37 \pm 0.60, \quad a_{2 K^{*}}^{0}=1.19 \pm 0.10$,

$a_{3 K^{*}}^{0}=-0.04 \pm 0.36$,

and the branching ratios in Table VII. It is seen that the central value of $a_{1 K^{*}}^{0}$ increases only a bit with an enlarged uncertainty and $a_{2 K^{*}}^{0}$ stays the same, compared with those from Scenario I in Table I, and the central value of $a_{3 K^{*}}^{0}$ is tiny. The corresponding branching ratios in Table VII also change very little, compared with those in Tables V and VI. The above observations support that the $S U(3)$-breaking effects in the considered modes can be explained by the $a_{1 K^{*}}^{0}$ term alone under the current data precision. Hence, the neglect of the $a_{3 K^{*}}^{0}$ term in this work is justified. Besides, it is not practical to include many parameters in the fit because of the limited amount of experimental data at present. For a similar reason, the asymptotic forms of the $K \pi$ twist- 3 DAs $\phi_{K \pi}^{s, t}$ are adopted in our analysis. The same argument applies to the expansion of the kaon DAs in Eq. (16), where the higher moments responsible for $S U(3)$-symmetry-breaking effects are also absent. We will explore the impact of these neglected Gegenbauer polynomials systematically in the future, when more experimental data with improved precision are available.

It is noticed that the parametrization of the parton momenta in Eqs. (2) and (3) introduces the dependence on the light meson mass $m_{3}$ into the hard kernels and the Sudakov exponents, as explicitly shown in the Appendix. Since both of these factors are perturbative pieces in a PQCD factorization formula, they should be insensitive to a light scale. Therefore, we test the sensitivity of our numerical results to this light scale by setting it to zero in the hard kernels and the Sudakov exponents. The corresponding branching ratios and direct $C P$ asymmetries for two typical modes, $B^{+} \rightarrow K^{+}\left(\rho^{0} \rightarrow\right) \pi \pi$ and $B^{0} \rightarrow \pi^{-}\left(K^{*+} \rightarrow\right) K \pi$ in Scenario II, are presented in Table VIII. The neglect of the kaon mass for the former mode causes about $10 \%$ variation in the branching ratio and the direct $C P$ asymmetry. The quantities associated with the latter mode are relatively

TABLE VIII. $\quad C P$ averaged branching ratios and direct $C P$ asymmetries of the $B^{+} \rightarrow K^{+}\left(\rho^{0} \rightarrow\right) \pi \pi$ decay and the $B^{0} \rightarrow \pi^{-}\left(K^{*+} \rightarrow\right) K \pi$ decay in Scenario II with and without the light meson masses in the hard kernels and the Sudakov exponents. The experimental data are quoted from Ref. [37]. The sources of the theoretical errors are the same as in Table II.

\begin{tabular}{|c|c|c|c|c|}
\hline Modes & & Results (with light mass) & Results (without light mass) & Data \\
\hline$B^{+} \rightarrow K^{+}\left(\rho^{0} \rightarrow\right) \pi \pi$ & $\begin{array}{l}\mathcal{B}\left(10^{-6}\right) \\
\mathcal{A}_{C P}(\%)\end{array}$ & $\begin{array}{c}2.91_{-0.60-0.68-0.82}^{+0.68+0.77+1.43} \\
53.5_{-1.4-4.5-11.9}^{+0.4+4.5}\end{array}$ & $\begin{array}{c}2.51_{-0.52-0.53-0.80}^{+0.56+0.71+1.34} \\
58.5_{-1.9-6.4+11.9-17.1}^{+0.0+4}\end{array}$ & $\begin{array}{c}3.7 \pm 0.5 \\
37 \pm 10\end{array}$ \\
\hline$B^{0} \rightarrow \pi^{-}\left(K^{*+} \rightarrow\right) K \pi$ & $\begin{array}{l}\mathcal{B}\left(10^{-6}\right) \\
\mathcal{A}_{C P}(\%)\end{array}$ & $\begin{array}{c}7.61_{-1.61-0.65-1.78}^{+1.83+0.92+2.40} \\
-32.3_{-0.2-8.4-6.3+7.3}^{+0.7+10.3+7.9}\end{array}$ & $\begin{array}{c}7.66_{-1.60-0.64-2.06}^{+1.84+0.65+2.43} \\
-32.7_{-0.1-8.4-6.4-10.1}^{+0.6+10.4+7.9}\end{array}$ & $\begin{aligned} 7.5 & \pm 0.4 \\
-27 & \pm 4\end{aligned}$ \\
\hline
\end{tabular}


TABLE IX. Fitted parameters for the $\omega^{2}$-dependent Gegenbauer moments in the twist- 2 and twist- $3 \pi \pi$ DAs.

\begin{tabular}{|c|c|c|c|c|c|c|}
\hline & $a_{2 \rho}^{0}$ & $a_{2 \rho}^{s}$ & $a_{2 \rho}^{t}$ & $c_{\rho}^{0}\left(\mathrm{GeV}^{-2}\right)$ & $c_{\rho}^{s}\left(\mathrm{GeV}^{-2}\right)$ & $c_{\rho}^{t}\left(\mathrm{GeV}^{-2}\right)$ \\
\hline Fit & $-0.45 \pm 0.29$ & $1.12 \pm 0.33$ & $-0.43 \pm 0.11$ & $-0.44 \pm 0.93$ & $-1.42 \pm 0.42$ & $-0.03 \pm 0.32$ \\
\hline
\end{tabular}

stable with respect to the neglect of the pion mass, as expected. The insensitivity to the light scale confirms that our parametrization for kinematic variables in three-body $B$-meson decays is reasonable.

\section{C. $\omega^{2}$-dependent Gegenbauer moments}

We make a more aggressive attempt in this subsection to determine the dependence of the Gegenbauer moments in the two-meson DAs on the meson-pair invariant mass. As stated in the Introduction, it is unlikely to extract the exact dependence from current data, so we simply expand the Gegenbauer moments up to the first power in $\omega^{2}$, and examine whether the additional linear terms can be constrained effectively in the global fit. Consider the parametrizations of the dipion DAs,

$$
\begin{aligned}
& \phi_{\pi \pi}^{0}\left(x, \omega^{2}\right)=\frac{3 F_{\pi \pi}^{\|}\left(\omega^{2}\right)}{\sqrt{2 N_{c}}} x(1-x)\left[1+a_{2 \rho}^{0}\left(1+c_{\rho}^{0} \omega^{2}\right) C_{2}^{3 / 2}(2 x-1)\right], \\
& \phi_{\pi \pi}^{s}\left(x, \omega^{2}\right)=\frac{3 F_{\pi \pi}^{\perp}\left(\omega^{2}\right)}{2 \sqrt{2 N_{c}}}(1-2 x)\left[1+a_{2 \rho}^{s}\left(1+c_{\rho}^{s} \omega^{2}\right)\left(10 x^{2}-10 x+1\right)\right], \\
& \phi_{\pi \pi}^{t}\left(x, \omega^{2}\right)=\frac{3 F_{\pi \pi}^{\perp}\left(\omega^{2}\right)}{2 \sqrt{2 N_{c}}}(1-2 x)^{2}\left[1+a_{2 \rho}^{t}\left(1+c_{\rho}^{t} \omega^{2}\right) C_{2}^{3 / 2}(2 x-1)\right],
\end{aligned}
$$

with the free parameters $a_{2 \rho}^{0, s, t}$ and $c_{\rho}^{0, s, t}$. The above parametrization follows the power series for the $\omega^{2}$-dependent Gegenbauer moments derived in Ref. [22].

The global fit to the same set of $B_{(s)} \rightarrow P(\rho \rightarrow) \pi \pi$ data with $P=\pi, K$ leads to the outcomes in Table IX with a

TABLE X. $C P$ averaged branching ratios and direct $C P$ asymmetries derived from the fitted Gegenbauer moments in Table IX, and compared with data [37]. For simplicity, only the theoretical errors from the Gegenbauer moments are presented.

\begin{tabular}{lccc}
\hline \hline Modes & & Results & Data \\
\hline$B^{+} \rightarrow K^{+}\left(\rho^{0} \rightarrow\right) \pi \pi$ & $\mathcal{B}\left(10^{-6}\right)$ & $3.12_{-1.14}^{+1.81}$ & $3.7 \pm 0.5^{\dagger}$ \\
& $\mathcal{A}_{C P}(\%)$ & $37.9_{-11.9}^{+10.6}$ & $37 \pm 10^{\dagger}$ \\
$B^{+} \rightarrow K^{0}\left(\rho^{+} \rightarrow\right) \pi \pi$ & $\mathcal{B}\left(10^{-6}\right)$ & $8.66_{-1.99}^{+3.24}$ & $7.3 \pm 1.2^{\dagger}$ \\
& $\mathcal{A}_{C P}(\%)$ & $17.8_{-1.1}^{+2.6}$ & $-3 \pm 15$ \\
$B^{0} \rightarrow K^{+}\left(\rho^{-} \rightarrow\right) \pi \pi$ & $\mathcal{B}\left(10^{-6}\right)$ & $8.22_{-1.93}^{+3.21}$ & $7.0 \pm 0.9^{\dagger}$ \\
& $\mathcal{A}_{C P}(\%)$ & $18.9_{-8.7}^{+7.8}$ & $20 \pm 11$ \\
$B^{0} \rightarrow K^{0}\left(\rho^{0} \rightarrow\right) \pi \pi$ & $\mathcal{B}\left(10^{-6}\right)$ & $2.88_{-0.72}^{+1.19}$ & $3.4 \pm 1.1^{\dagger}$ \\
& $\mathcal{A}_{C P}(\%)$ & $1.9_{-0.8}^{+1.8}$ & $-4 \pm 20$ \\
$B^{+} \rightarrow \pi^{+}\left(\rho^{0} \rightarrow\right) \pi \pi$ & $\mathcal{B}\left(10^{-6}\right)$ & $7.69_{-1.65}^{+2.67}$ & $8.3 \pm 1.2^{\dagger}$ \\
& $\mathcal{A}_{C P}(\%)$ & $-17.2_{-4.2}^{+9.2}$ & $0.9 \pm 1.9$ \\
$B^{+} \rightarrow \pi^{0}\left(\rho^{+} \rightarrow\right) \pi \pi$ & $\mathcal{B}\left(10^{-6}\right)$ & $10.14_{-3.89}^{+5.18}$ & $10.9 \pm 1.4^{\dagger}$ \\
& $\mathcal{A}_{C P}(\%)$ & $5.6_{-14.4}^{+13.4}$ & $2 \pm 11$ \\
$B^{0} \rightarrow \pi^{-}\left(\rho^{+} \rightarrow\right) \pi \pi$ & $\mathcal{B}\left(10^{-6}\right)$ & $24.49_{-1.72}^{+2.29}$ & $23.0 \pm 2.3^{1 \dagger}$ \\
& $\mathcal{A}_{C P}(\%)$ & $3.8_{-5.3}^{+5.1}$ & $13 \pm 6$ \\
$B^{0} \rightarrow \pi^{+}\left(\rho^{-} \rightarrow\right) \pi \pi$ & $\mathcal{B}\left(10^{-6}\right)$ & $24.49_{-1.72}^{+2.29}$ & $23.0 \pm 2.3^{1 \dagger}$ \\
& $\mathcal{A}_{C P}(\%)$ & $-16.4_{-10.1}^{+11.8}$ & $-8 \pm 8$ \\
\hline \hline
\end{tabular}

smaller $\chi^{2} /$ d.o.f. $=0.51$, which are not difficult to understand: varying $\omega^{2}$ around the $\rho$ resonance in its width window, we find that the values of $a_{2 \rho}^{0, s, t}\left(1+c_{\rho}^{0, s, t} \omega^{2}\right)$ are in fact consistent with the corresponding ones in Table I. The consistency is particularly obvious for $a_{2 \rho}^{t}\left(1+c_{\rho}^{t} \omega^{2}\right)$ with the tiny coefficient $c_{\rho}^{t}$. It is observed from Table IX that the parameters for the twist-3 DA $\phi_{\pi \pi}^{s}$, which gives sizable contributions to branching ratios, can be constrained effectively by the current data. It suggests that the determination of the $\omega^{2}$-dependent Gegenbauer moments is promising, when more precise data are available in the future. Because our purpose is to demonstrate the potential to extract the $\omega^{2}$ dependence of the Gegenbauer moments, we will not work on the $K \pi$ and $K K$ DAs. The effect of including the $\omega^{2}$ dependence of the Gegenbauer moments is similar to that of introducing more parameters. That is, the fit quality is improved with a lower $\chi^{2} /$ d.o.f. at the cost of larger uncertainties for fit results as shown in Table X. For example, the reproduced branching ratios for the $B^{+} \rightarrow$ $K^{+}\left(\rho^{0} \rightarrow\right) \pi \pi$ and $B^{+} \rightarrow \pi^{+}\left(\rho^{0} \rightarrow\right) \pi \pi$ decays get closer to the data, which have relatively higher precision. However, the uncertainty caused by the variation of the dipion DAs is amplified compared to the second source of errors in Table II.

\section{CONCLUSION}

In this work we have performed a global fit of the Gegenbauer moments in two-meson DAs to measured 
branching ratios and direct $C P$ asymmetries in the threebody hadronic $B$-meson decays $B \rightarrow V P_{3} \rightarrow P_{1} P_{2} P_{3}$, with $V=\rho, \phi, K^{*}$ and $P_{3}=\pi, K$, in the LO PQCD approach. Two-meson DAs, collecting both nonresonant and multiresonance contributions, serve as crucial nonperturbative ingredients of factorization theorems for the above decays. The Gegenbauer moments of the pion and kaon DAs determined in the LO global analysis of two-body hadronic $B$-meson decays have been input for theoretical consistency. To facilitate the numerical study, we have constructed a Gegenbauer-moment-independent database, via which a decay amplitude is decomposed into a linear combination of the relevant Gegenbauer moments in the two-meson DAs. It was noticed that the fitted Gegenbauer moments differ from those associated with an intermediate resonance which decays into the meson pair, and from those adopted in previous PQCD calculations. This observation indicates that the Gegenbauer moments of a two-meson DA cannot be inferred from sum-rule results for an intermediate resonance, and their global determination is essential.

We have examined two scenarios for the determination of the $K \pi$ DAs in order to check the convergence of the Gegenbauer expansion, and the sensitivity of the fitted observables to our setup. It was found that the Gegenbauer expansion is improved by increasing the number of Gegenbauer moments at the cost of large uncertainties for fit outcomes, and that the branching ratios of $B_{s}$-meson decays and direct $C P$ asymmetries in some modes are more sensitive to the chosen scenarios. Hence, more accurate $K \pi$ DAs are necessary to unambiguously predict these quantities. We state that our fits have not been able to discriminate the two scenarios effectively. We have also explored the potential to fix the dependence of the Gegenbauer moments on the meson-pair invariant mass, and confirmed that at least the parameter for the twist-3 DA $\phi_{\pi \pi}^{s}$ can be constrained to some extent by the current data. Therefore, the determination of the dependence on the meson-pair invariant mass is promising, when data become more precise.

We mention that the three-body charmless hadronic $B$-meson decays included in this work have been studied in Refs. $[6,7,10,11]$ in a scattered manner. The improvements compared to the earlier studies are as follows: (1) the partonic kinematic variables have been refined to take into account finite masses of final-state mesons, such that the $S U(3)$-symmetry-breaking effects in the decays can be evaluated more precisely; (2) the Gegenbauer moments in the two-meson DAs have been determined in a global analysis for the first time, which are valuable for future applications of the PQCD framework to multibody $B$-meson decays; and (3) the dependence of the Gegenbauer moments on the meson-pair invariant mass has been probed for the first time. Because of (1), the numerous hard kernels involved in the various modes need to be modified, which are presented, together with the factorization formulas for the decay amplitudes, in the Appendix. The refined partonic kinematics is general enough for its extension to multibody $B$-meson decays into arbitrary massive final states. For (2), we recall that different Gegenbauer moments for the $K \pi$ DAs were taken in the previous scattered studies, such as Refs. [7,11], so our work facilitates a consistent understanding of multibody $B$-meson decays. We have shown that the preferred central value of, for instance, the Gegenbauer moment $a_{1 K^{*}}^{0}$ is 0.31 , instead of 0.2 in Ref. [7] or 0.05 in Ref. [11] (but note the large theoretical uncertainties).

It has been demonstrated that most of the data considered in the fit are well reproduced, namely, the fit quality is satisfactory. It implies that the two-meson DAs presented in this paper are ready for applications to other multibody hadronic $B$-meson decays involving the same meson pairs. With the obtained Gegenbauer moments, we have made predictions for those observables, whose data were excluded in the fit because of their substantial experimental errors or significant subleading contributions to the corresponding factorization formulas. Except for the $B_{s}^{0} \rightarrow$ $\pi^{+}\left(K^{*-} \rightarrow\right) K \pi$ branching ratio, our predictions agree with the data within uncertainties in the former case. Since our results were still derived in the LO PQCD approach, the data in the latter case remain unexplained, and deserve more through investigations. As pointed out before, the precision of the extracted two-meson DAs can be improved systematically, when higher-order and/or higher-power corrections to three-body hadronic $B$-meson decays are taken into account in our formalism. At the same time, more precise measurements are encouraged, especially those of $C P$ asymmetries. These efforts will strengthen the constraint on the Gegenbauer moments and sharpen the confrontation between theoretical predictions and experimental data.

\section{ACKNOWLEDGMENTS}

We thank Wen-Fei Wang for helpful discussions. This work is supported in part by "Fundamental Research Funds for Central Universities" under Grant No. KJQN202144 and the National Natural Science Foundation of China under Grant Nos. 12005103, 12075086, 11947013, 11947215 and U2032102, and by MOST of R.O.C. under Grant No. MOST-107-2119-M-001-035-MY3. Y. L. is also supported by the Natural Science Foundation of Jiangsu Province under Grant No. BK20190508 and the Research Start-up Funds of Nanjing Agricultural University. D.-C. Y. is supported by the Natural Science Foundation of Jiangsu Province under Grant No. BK20200980. Z. R. is supported in part by the Natural Science Foundation of Hebei Province under Grant Nos. A2019209449 and A2021209002. 


\section{APPENDIX: DECAY AMPLITUDES}

In this appendix we present the PQCD factorization formulas for the amplitudes of the considered three-body charmless hadronic $B$-meson decays. We first decompose various decay amplitudes $\mathcal{A}$ in terms of the factorizable emission (annihilation) contributions $F_{e(a) V}$ and the nonfactorizable emission (annihilation) contributions $M_{e(a) V}$ for the intermediate vector mesons $V=\rho, K^{*}, \phi$ from Fig. 1, and the similar ones $F_{e(a) P}$ and $M_{e(a) P}$ for the bachelor mesons $P=\pi, K$ from Fig. 2. These contributions are further labeled by the superscripts $L L, L R$, and $S P$ corresponding to the $(V-A)(V-A)$, $(V-A)(V+A)$, and $(S-P)(S+P)$ operators, respectively:

(1) $B_{(s)} \rightarrow K(\rho \rightarrow) \pi \pi$

$$
\begin{aligned}
& \mathcal{A}\left(B^{+} \rightarrow K^{+}\left(\rho^{0} \rightarrow\right) \pi \pi\right)=\frac{G_{F}}{2}\left\{V_{u b}^{*} V_{u s}\left[\left(\frac{C_{1}}{3}+C_{2}\right)\left(F_{e \rho}^{L L}+F_{a \rho}^{L L}\right)+\left(C_{1}+\frac{C_{2}}{3}\right) F_{e K}^{L L}+C_{2} M_{e K}^{L L}+C_{1}\left(M_{e \rho}^{L L}+M_{a \rho}^{L L}\right)\right]\right. \\
& -V_{t b}^{*} V_{t s}\left[\left(\frac{C_{3}}{3}+C_{4}+\frac{C_{9}}{3}+C_{10}\right)\left(F_{e \rho}^{L L}+F_{a \rho}^{L L}\right)\right. \\
& +\left(\frac{C_{5}}{3}+C_{6}+\frac{C_{7}}{3}+C_{8}\right)\left(F_{e \rho}^{S P}+F_{a \rho}^{S P}\right)+\left(C_{3}+C_{9}\right)\left(M_{e \rho}^{L L}+M_{a \rho}^{L L}\right) \\
& +\left(C_{5}+C_{7}\right)\left(M_{e \rho}^{L R}+M_{a \rho}^{L R}\right)+\frac{3 C_{8}}{2} M_{e K}^{S P}+\frac{3 C_{10}}{2} M_{e K}^{L L} \\
& \left.\left.+\frac{3}{2}\left(C_{7}+\frac{C_{8}}{3}+C_{9}+\frac{C_{10}}{3}\right) F_{e K}^{L L}\right]\right\} \\
& \mathcal{A}\left(B^{0} \rightarrow K^{+}\left(\rho^{-} \rightarrow\right) \pi \pi\right)=\frac{G_{F}}{\sqrt{2}}\left\{V_{u b}^{*} V_{u s}\left[\left(\frac{C_{1}}{3}+C_{2}\right) F_{e \rho}^{L L}+C_{1} M_{e \rho}^{L L}\right]\right. \\
& -V_{t b}^{*} V_{t s}\left[\left(C_{3}+C_{9}\right) M_{e \rho}^{L L}+\left(\frac{C_{3}}{3}+C_{4}+\frac{C_{9}}{3}+C_{10}\right) F_{e \rho}^{L L}\right. \\
& +\left(\frac{C_{5}}{3}+C_{6}+\frac{C_{7}}{3}+C_{8}\right) F_{e \rho}^{S P}+\left(C_{5}+C_{7}\right) M_{e \rho}^{L R}+\left(\frac{C_{3}}{3}+C_{4}-\frac{1}{2}\left(\frac{C_{9}}{3}+C_{10}\right)\right) F_{a \rho}^{L L} \\
& \left.\left.+\left(C_{3}-\frac{C_{9}}{2}\right) M_{a \rho}^{L L}+\left(\frac{C_{5}}{3}+C_{6}-\frac{1}{2}\left(\frac{C_{7}}{3}+C_{8}\right)\right) F_{a \rho}^{S P}+\left(C_{5}-\frac{C_{7}}{2}\right) M_{a \rho}^{L R}\right]\right\}, \\
& \mathcal{A}\left(B_{s}^{0} \rightarrow K^{-}\left(\rho^{+} \rightarrow\right) \pi \pi\right)=\frac{G_{F}}{\sqrt{2}}\left\{V_{u b}^{*} V_{u d}\left[\left(\frac{C_{1}}{3}+C_{2}\right) F_{e K}^{L L}+C_{1} M_{e K}^{L L}\right]\right. \\
& -V_{t b}^{*} V_{t d}\left[\left(C_{3}+C_{9}\right) M_{e K}^{L L}+\left(\frac{C_{3}}{3}+C_{4}+\frac{C_{9}}{3}+C_{10}\right) F_{e K}^{L L}+\left(C_{5}+C_{7}\right) M_{e K}^{L R}\right. \\
& +\left(\frac{C_{3}}{3}+C_{4}-\frac{1}{2}\left(\frac{C_{9}}{3}+C_{10}\right)\right) F_{a K}^{L L}+\left(\frac{C_{5}}{3}+C_{6}-\frac{1}{2}\left(\frac{C_{7}}{3}+C_{8}\right)\right) F_{a K}^{S P} \\
& \left.\left.+\left(C_{3}-\frac{C_{9}}{2}\right) M_{a K}^{L L}+\left(C_{5}-\frac{C_{7}}{2}\right) M_{a K}^{L R}\right]\right\} \\
& \mathcal{A}\left(B^{+} \rightarrow K^{0}\left(\rho^{+} \rightarrow\right) \pi \pi\right)=\frac{G_{F}}{\sqrt{2}}\left\{V_{u b}^{*} V_{u s}\left[\left(\frac{C_{1}}{3}+C_{2}\right) F_{a \rho}^{L L}+C_{1} M_{a \rho}^{L L}\right]-V_{t b}^{*} V_{t s}\left[\left(C_{3}-\frac{C_{9}}{2}\right) M_{e \rho}^{L L}\right.\right. \\
& +\left(\frac{C_{3}}{3}+C_{4}-\frac{1}{2}\left(\frac{C_{9}}{3}+C_{10}\right)\right) F_{e \rho}^{L L}+\left(\frac{C_{5}}{3}+C_{6}-\frac{1}{2}\left(\frac{C_{7}}{3}+C_{8}\right)\right) F_{e \rho}^{S P} \\
& +\left(C_{5}-\frac{C_{7}}{2}\right) M_{e \rho}^{L R}+\left(\frac{C_{3}}{3}+C_{4}+\frac{C_{9}}{3}+C_{10}\right) F_{a \rho}^{L L}+\left(C_{3}+C_{9}\right) M_{a \rho}^{L L} \\
& \left.\left.+\left(\frac{C_{5}}{3}+C_{6}+\frac{C_{7}}{3}+C_{8}\right) F_{a \rho}^{S P}+\left(C_{5}+C_{7}\right) M_{a \rho}^{L R}\right]\right\}
\end{aligned}
$$




$$
\begin{aligned}
\mathcal{A}\left(B^{0} \rightarrow K^{0}\left(\rho^{0} \rightarrow\right) \pi \pi\right)= & \frac{G_{F}}{2}\left\{V_{u b}^{*} V_{u s}\left[\left(C_{1}+\frac{C_{2}}{3}\right) F_{e K}^{L L}+C_{2} M_{e K}^{L L}\right]-V_{t b}^{*} V_{t s}\left[\frac{3 C_{8}}{2} M_{e K}^{S P}\right.\right. \\
& -\left(\frac{C_{3}}{3}+C_{4}-\frac{1}{2}\left(\frac{C_{9}}{3}+C_{10}\right)\right)\left(F_{e \rho}^{L L}+F_{a \rho}^{L L}\right)-\left(C_{3}-\frac{C_{9}}{2}\right)\left(M_{e \rho}^{L L}+M_{a \rho}^{L L}\right) \\
& -\left(\frac{C_{5}}{3}+C_{6}-\frac{1}{2}\left(\frac{C_{7}}{3}+C_{8}\right)\right)\left(F_{e \rho}^{S P}+F_{a \rho}^{S P}\right)-\left(C_{5}-\frac{C_{7}}{2}\right)\left(M_{e \rho}^{L R}+M_{a \rho}^{L R}\right) \\
& \left.\left.+\frac{3}{2}\left(C_{7}+\frac{C_{8}}{3}+C_{9}+\frac{C_{10}}{3}\right) F_{e K}^{L L}+\frac{3 C_{10}}{2} M_{e K}^{L L}\right]\right\}, \\
\mathcal{A}\left(B_{s}^{0} \rightarrow K^{0}\left(\rho^{0} \rightarrow\right)\right. & \pi \pi)=\frac{G_{F}}{2}\left\{V_{u b}^{*} V_{u d}\left[\left(C_{1}+\frac{C_{2}}{3}\right) F_{e K}^{L L}+C_{2} M_{e K}^{L L}\right]-V_{t b}^{*} V_{t d}\left[\frac{3 C_{8}}{2} M_{e K}^{S P}\right.\right. \\
& +\left(-\frac{C_{3}}{3}-C_{4}+\frac{5 C_{9}}{3}+C_{10}+\frac{3}{2}\left(C_{7}+\frac{C_{8}}{3}\right)\right) F_{e K}^{L L} \\
& +\left(-C_{3}+\frac{C_{9}}{2}+\frac{3 C_{10}}{2}\right) M_{e K}^{L L}-\left(C_{5}-\frac{C_{7}}{2}\right)\left(M_{e K}^{L R}+M_{a K}^{L R}\right) \\
& \left.\left.\left.\left.-\left(\frac{C_{3}}{3}+C_{4}-\frac{1}{2}\left(\frac{C_{9}}{3}+C_{10}\right)\right) F_{a K}^{L L}\right)-\left(C_{3}-\frac{C_{9}}{2}\right) M_{a K}^{L L}+C_{6}-\frac{1}{2}\left(\frac{C_{7}}{3}+C_{8}\right)\right) F_{a K}^{S P}\right]\right\} .
\end{aligned}
$$

(2) $B_{(s)} \rightarrow \pi(\rho \rightarrow) \pi \pi$

$$
\begin{aligned}
& \mathcal{A}\left(B^{+} \rightarrow \pi^{+}\left(\rho^{0} \rightarrow\right) \pi \pi\right)=\frac{G_{F}}{2}\left\{V _ { u b } ^ { * } V _ { u d } \left[\left(\frac{C_{1}}{3}+C_{2}\right)\left(F_{e \rho}^{L L}+F_{a \rho}^{L L}-F_{a \pi}^{L L}\right)+\left(C_{1}+\frac{C_{2}}{3}\right) F_{e \pi}^{L L}\right.\right. \\
& \left.+C_{1}\left(M_{e \rho}^{L L}+M_{a \rho}^{L L}-M_{a \pi}^{L L}\right)+C_{2} M_{e \pi}^{L L}\right] \\
& -V_{t b}^{*} V_{t d}\left[\frac{3 C_{8}}{2} M_{e \pi}^{S P}+\left(\frac{C_{3}}{3}+C_{4}+\frac{C_{9}}{3}+C_{10}\right)\left(F_{e \rho}^{L L}+F_{a \rho}^{L L}-F_{a \pi}^{L L}\right)\right. \\
& +\left(C_{3}+C_{9}\right)\left(M_{e \rho}^{L L}+M_{a \rho}^{L L}-M_{a \pi}^{L L}\right)+\left(-C_{5}+\frac{C_{7}}{2}\right) M_{e \pi}^{L R} \\
& +\left(\frac{C_{5}}{3}+C_{6}+\frac{C_{7}}{3}+C_{8}\right)\left(F_{e \rho}^{S P}+F_{a \rho}^{S P}-F_{a \pi}^{S P}\right)+\left(C_{5}+C_{7}\right)\left(M_{e \rho}^{L R}+M_{a \rho}^{L R}-M_{a \pi}^{L R}\right) \\
& \left.\left.+\left(-\frac{C_{3}}{3}-C_{4}+\frac{5}{3} C_{9}+C_{10}+\frac{3}{2}\left(C_{7}+\frac{C_{8}}{3}\right)\right) F_{e \pi}^{L L}+\left(-C_{3}+\frac{C_{9}}{2}+\frac{3 C_{10}}{2}\right) M_{e \pi}^{L L}\right]\right\}, \\
& \mathcal{A}\left(B^{0} \rightarrow \pi^{-}\left(\rho^{+} \rightarrow\right) \pi \pi\right)=\frac{G_{F}}{\sqrt{2}}\left\{V_{u b}^{*} V_{u d}\left[\left(C_{1}+\frac{C_{2}}{3}\right) F_{a \rho}^{L L}+\left(\frac{C_{1}}{3}+C_{2}\right) F_{e \pi}^{L L}+C_{2} M_{a \rho}^{L L}+C_{1} M_{e \pi}^{L L}\right]\right. \\
& -V_{t b}^{*} V_{t d}\left[\left(\frac{C_{3}}{3}+C_{4}+\frac{C_{9}}{3}+C_{10}\right) F_{e \pi}^{L L}+\left(C_{4}+C_{10}\right) M_{a \rho}^{L L}+\left(C_{3}+\frac{C_{4}}{3}-C_{5}-\frac{C_{6}}{3}-C_{7}-\frac{C_{8}}{3}\right.\right. \\
& \left.+C_{9}+\frac{C_{10}}{3}\right) F_{a \rho}^{L L}+\left(C_{3}+C_{9}\right) M_{e \pi}^{L L}+\left(C_{5}+C_{7}\right) M_{e \pi}^{L R}+\left(C_{5}-\frac{C_{7}}{2}\right) M_{a \pi}^{L R} \\
& +\left(\frac{4}{3}\left(C_{3}+C_{4}-\frac{C_{9}}{2}-\frac{C_{10}}{2}\right)-C_{5}-\frac{C_{6}}{3}+\frac{1}{2}\left(C_{7}+\frac{C_{8}}{3}\right)\right) F_{a \pi}^{L L}+\left(\frac{C_{5}}{3}+C_{6}-\frac{1}{2}\left(\frac{C_{7}}{3}+C_{8}\right)\right) F_{a \pi}^{S P} \\
& \left.\left.+\left(C_{6}-\frac{C_{8}}{2}\right) M_{a \pi}^{S P}+\left(C_{3}+C_{4}-\frac{C_{9}}{2}-\frac{C_{10}}{2}\right) M_{a \pi}^{L L}+\left(C_{6}+C_{8}\right) M_{a \rho}^{S P}\right]\right\},
\end{aligned}
$$




$$
\begin{aligned}
\mathcal{A}\left(B^{0} \rightarrow \pi^{+}\left(\rho^{-} \rightarrow\right) \pi \pi\right)= & \frac{G_{F}}{\sqrt{2}}\left\{V_{u b}^{*} V_{u d}\left[\left(\frac{C_{1}}{3}+C_{2}\right) F_{e \rho}^{L L}+\left(C_{1}+\frac{C_{2}}{3}\right) F_{a \pi}^{L L}+C_{1} M_{e \rho}^{L L}+C_{2} M_{a \pi}^{L L}\right]\right. \\
& -V_{t b}^{*} V_{t d}\left[\left(\frac{C_{3}}{3}+C_{4}+\frac{C_{9}}{3}+C_{10}\right) F_{e \rho}^{L L}+\left(C_{3}+C_{9}\right) M_{e \rho}^{L L}+\left(\frac{C_{5}}{3}+C_{6}+\frac{C_{7}}{3}+C_{8}\right) F_{e \rho}^{S P}\right. \\
& +\left(C_{5}+C_{7}\right) M_{e \rho}^{L R}+\left(C_{6}+C_{8}\right) M_{a \pi}^{S P}+\left(\frac{4}{3}\left(C_{3}+C_{4}-\frac{C_{9}}{2}-\frac{C_{10}}{2}\right)-C_{5}-\frac{C_{6}}{3}\right. \\
+ & \left.\frac{1}{2}\left(C_{7}+\frac{C_{8}}{3}\right)\right) F_{a \rho}^{L L}+\left(\frac{C_{5}}{3}+C_{6}-\frac{1}{2}\left(\frac{C_{7}}{3}+C_{8}\right)\right) F_{a \rho}^{S P} \\
+ & \left(C_{3}+C_{4}-\frac{C_{9}}{2}-\frac{C_{10}}{2}\right) M_{a \rho}^{L L}+\left(C_{5}-\frac{C_{7}}{2}\right) M_{a \rho}^{L R}+\left(C_{6}-\frac{C_{8}}{2}\right) M_{a \rho}^{S P}+\left(C_{4}+C_{10}\right) M_{a \pi}^{L L} \\
+ & \left.\left.\left(C_{3}+\frac{C_{4}}{3}-C_{5}-\frac{C_{6}}{3}-C_{7}-\frac{C_{8}}{3}+C_{9}+\frac{C_{10}}{3}\right) F_{a \pi}^{L L}\right]\right\} \\
\mathcal{A}\left(B_{s}^{0} \rightarrow \pi^{-}\left(\rho^{+} \rightarrow\right) \pi\right. & =\frac{G_{F}}{\sqrt{2}}\left\{V_{u b}^{*} V_{u s}\left[\left(C_{1}+\frac{C_{2}}{3}\right) F_{a \rho}^{L L}+C_{2} M_{a \rho}^{L L}\right]-V_{t b}^{*} V_{t s}\left[\left(C_{6}+C_{8}\right) M_{a \rho}^{S P}\right.\right. \\
& +\left(C_{3}+\frac{C_{4}}{3}-C_{5}-\frac{C_{6}}{3}-C_{7}-\frac{C_{8}}{3}+C_{9}+\frac{C_{10}}{3}\right) F_{a \rho}^{L L} \\
& +\left(C_{3}+\frac{C_{4}}{3}-\frac{1}{2}\left(C_{9}+\frac{C_{10}}{3}\right)-C_{5}-\frac{C_{6}}{3}+\frac{1}{2}\left(C_{7}+\frac{C_{8}}{3}\right)\right) F_{a \pi}^{L L} \\
& \left.\left.+\left(C_{4}-\frac{C_{10}}{2}\right) M_{a \pi}^{L L}+\left(C_{6}-\frac{C_{8}}{2}\right) M_{a \pi}^{S P}+\left(C_{4}+C_{10}\right) M_{a \rho}^{L L}\right]\right\},
\end{aligned}
$$

$$
\begin{aligned}
\mathcal{A}\left(B_{s}^{0} \rightarrow \pi^{+}\left(\rho^{-} \rightarrow\right) \pi \pi\right)= & \frac{G_{F}}{\sqrt{2}}\left\{V_{u b}^{*} V_{u s}\left[\left(C_{1}+\frac{C_{2}}{3}\right) F_{a \pi}^{L L}+C_{2} M_{a \pi}^{L L}\right]-V_{t b}^{*} V_{t s}\left[\left(C_{4}-\frac{C_{10}}{2}\right) M_{a \rho}^{L L}\right.\right. \\
& +\left(C_{3}+\frac{C_{4}}{3}-\frac{1}{2}\left(C_{9}+\frac{C_{10}}{3}\right)-C_{5}-\frac{C_{6}}{3}+\frac{1}{2}\left(C_{7}+\frac{C_{8}}{3}\right)\right) F_{a \rho}^{L L}+\left(C_{6}-\frac{C_{8}}{2}\right) M_{a \rho}^{S P} \\
& \left.\left.+\left(C_{4}+C_{10}\right) M_{a \pi}^{L L}+\left(C_{6}+C_{8}\right) M_{a \pi}^{S P}+\left(C_{3}+\frac{C_{4}}{3}-C_{5}-\frac{C_{6}}{3}-C_{7}-\frac{C_{8}}{3}+C_{9}+\frac{C_{10}}{3}\right) F_{a \pi}^{L L}\right]\right\},
\end{aligned}
$$

$$
\begin{aligned}
\mathcal{A}\left(B^{+} \rightarrow \pi^{0}\left(\rho^{+} \rightarrow\right) \pi \pi\right)= & \frac{G_{F}}{2}\left\{V _ { u b } ^ { * } V _ { u d } \left[\left(C_{1}+\frac{C_{2}}{3}\right) F_{e \rho}^{L L}+\left(\frac{C_{1}}{3}+C_{2}\right)\left(-F_{a \rho}^{L L}+F_{e \pi}^{L L}+F_{a \pi}^{L L}\right)\right.\right. \\
& \left.+C_{2} M_{e \rho}^{L L}+C_{1}\left(-M_{a \rho}^{L L}+M_{e \pi}^{L L}+M_{a \pi}^{L L}\right)\right]-V_{t b}^{*} V_{t d}\left[\frac{3 C_{8}}{2} M_{e \rho}^{S P}\right. \\
& +\left(-\frac{C_{3}}{3}-C_{4}-\frac{3}{2}\left(C_{7}+\frac{C_{8}}{3}\right)+\frac{5 C_{9}}{3}+C_{10}\right) F_{e \rho}^{L L} \\
& +\left(-\frac{C_{5}}{3}-C_{6}+\frac{1}{2}\left(\frac{C_{7}}{3}+C_{8}\right)\right) F_{e \rho}^{S P}+\left(-C_{3}+\frac{C_{9}}{2}+\frac{3 C_{10}}{2}\right) M_{e \rho}^{L L} \\
& +\left(\frac{C_{3}}{3}+C_{4}+\frac{C_{9}}{3}+C_{10}\right)\left(-F_{a \rho}^{L L}+F_{e \pi}^{L L}+F_{a \pi}^{L L}\right) \\
& +\left(\frac{C_{5}}{3}+C_{6}+\frac{C_{7}}{3}+C_{8}\right)\left(-F_{a \rho}^{S P}+F_{a \pi}^{S P}\right)+\left(-C_{5}+\frac{C_{7}}{2}\right) M_{e \rho}^{L R} \\
& \left.\left.+\left(C_{3}+C_{9}\right)\left(-M_{a \rho}^{L L}+M_{e \pi}^{L L}+M_{a \pi}^{L L}\right)+\left(C_{5}+C_{7}\right)\left(-M_{a \rho}^{L R}+M_{e \pi}^{L R}+M_{a \pi}^{L R}\right)\right]\right\}
\end{aligned}
$$




$$
\begin{aligned}
\mathcal{A}\left(B^{0} \rightarrow \pi^{0}\left(\rho^{0} \rightarrow\right) \pi \pi\right)= & -\frac{G_{F}}{2 \sqrt{2}}\left\{V_{u b}^{*} V_{u d}\left[\left(C_{1}+\frac{C_{2}}{3}\right)\left(F_{e \rho}^{L L}-F_{a \rho}^{L L}+F_{e \pi}^{L L}-F_{a \pi}^{L L}\right)+C_{2}\left(M_{e \rho}^{L L}-M_{a \rho}^{L L}+M_{e \pi}^{L L}-M_{a \pi}^{L L}\right)\right]\right. \\
& -V_{t b}^{*} V_{t d}\left[\frac{3 C_{8}}{2}\left(M_{e \rho}^{S P}+M_{e \pi}^{S P}\right)+\left(-\frac{C_{3}}{3}-C_{4}-\frac{3}{2}\left(C_{7}+\frac{C_{8}}{3}\right)+\frac{5 C_{9}}{3}+C_{10}\right)\left(F_{e \rho}^{L L}+F_{e \pi}^{L L}\right)\right. \\
& +\left(-\frac{C_{5}}{3}-C_{6}+\frac{1}{2}\left(\frac{C_{7}}{3}+C_{8}\right)\right) F_{e \rho}^{S P}+\left(-C_{3}+\frac{C_{9}}{2}+\frac{3 C_{10}}{2}\right)\left(M_{e \rho}^{L L}+M_{e \pi}^{L L}\right) \\
& +\left(-C_{5}+\frac{C_{7}}{2}\right)\left(M_{e \rho}^{L R}+M_{e \pi}^{L L}\right)-\left(2 C_{6}+\frac{C_{8}}{2}\right)\left(M_{a \rho}^{S P}+M_{a \pi}^{S P}\right)-\left(\frac{7 C_{3}}{3}+\frac{5 C_{4}}{3}-2\left(C_{5}+\frac{C_{6}}{3}\right)\right. \\
& \left.-\frac{1}{2}\left(C_{7}+\frac{C_{8}}{3}-\frac{2}{3}\left(C_{9}-C_{10}\right)\right)\right)\left(F_{a \rho}^{L L}+F_{a \pi}^{L L}\right)-\left(\frac{C_{5}}{3}+C_{6}-\frac{1}{2}\left(\frac{C_{7}}{3}+C_{8}\right)\right)\left(F_{a \rho}^{S P}+F_{a \pi}^{S P}\right) \\
& \left.\left.-\left(C_{5}-\frac{C_{7}}{2}\right)\left(M_{a \rho}^{L R}+M_{a \pi}^{L R}\right)-\left(C_{3}+2 C_{4}-\frac{C_{9}}{2}+\frac{C_{10}}{2}\right)\left(M_{a \rho}^{L L}+M_{a \pi}^{L L}\right)\right]\right\}, \\
\mathcal{A}\left(B_{s}^{0} \rightarrow \pi^{0}\left(\rho^{0} \rightarrow\right) \pi \pi\right)= & \frac{G_{F}}{2 \sqrt{2}}\left\{V_{u b}^{*} V_{u s}\left[\left(C_{1}+\frac{C_{2}}{3}\right)\left(F_{a \rho}^{L L}+F_{a \pi}^{L L}\right)+C_{2}\left(M_{a \rho}^{L L}+M_{a \pi}^{L L}\right)\right]\right. \\
& -V_{t b}^{*} V_{t s}\left[\left(2\left(C_{3}+\frac{C_{4}}{3}-C_{5}-\frac{C_{6}}{3}\right)-\frac{1}{2}\left(C_{7}+\frac{C_{8}}{3}-C_{9}-\frac{C_{10}}{3}\right)\right)\left(F_{a \rho}^{L L}+F_{a \pi}^{L L}\right)\right. \\
& \left.\left.+\left(2 C_{4}+\frac{C_{10}}{2}\right)\left(M_{a \rho}^{L L}+M_{a \pi}^{L L}\right)+\left(2 C_{6}+\frac{C_{8}}{2}\right)\left(M_{a \rho}^{S P}+M_{a \pi}^{S P}\right)\right]\right\} .
\end{aligned}
$$

(3) $B_{(s)} \rightarrow K\left(K^{*} \rightarrow\right) K \pi$

$$
\begin{aligned}
& \mathcal{A}\left(B^{+} \rightarrow K^{+}\left(\bar{K}^{* 0} \rightarrow\right) K \pi\right)=\frac{G_{F}}{\sqrt{2}}\left\{V_{u b}^{*} V_{u d}\left[\left(\frac{C_{1}}{3}+C_{2}\right) F_{a K}^{L L}+C_{1} M_{a K}^{L L}\right]-V_{t b}^{*} V_{t d}\left[\left(\frac{C_{3}}{3}+C_{4}-\frac{C_{9}}{6}-\frac{C_{10}}{2}\right) F_{e K}^{L L}\right.\right. \\
& +\left(C_{3}-\frac{C_{9}}{2}\right) M_{e K}^{L L}+\left(C_{5}-\frac{C_{7}}{2}\right) M_{e K}^{L R}+\left(\frac{C_{3}}{3}+C_{4}+\frac{C_{9}}{3}+C_{10}\right) F_{a K}^{L L} \\
& \left.\left.+\left(\frac{C_{5}}{3}+C_{6}+\frac{C_{7}}{3}+C_{8}\right) F_{a K}^{S P}+\left(C_{3}+C_{9}\right) M_{a K}^{L L}+\left(C_{5}+C_{7}\right) M_{a K}^{L R}\right]\right\} \\
& \mathcal{A}\left(B^{0} \rightarrow K^{+}\left(K^{*-} \rightarrow\right) K \pi\right)=\frac{G_{F}}{\sqrt{2}}\left\{V_{u b}^{*} V_{u d}\left[\left(C_{1}+\frac{C_{2}}{3}\right) F_{a K}^{L L}+C_{2} M_{a K}^{L L}\right]\right. \\
& -V_{t b}^{*} V_{t d}\left[\left(C_{3}+\frac{C_{4}}{3}-\frac{C_{9}}{2}-\frac{C_{10}}{6}-C_{5}-\frac{C_{6}}{3}+\frac{C_{7}}{2}+\frac{C_{8}}{6}\right) F_{a K^{*}}^{L L}\right. \\
& +\left(C_{4}-\frac{C_{10}}{2}\right) M_{a K^{*}}^{L L}+\left(C_{6}-\frac{C_{8}}{2}\right) M_{a K^{*}}^{S P}+\left(C_{3}+\frac{C_{4}}{3}+C_{9}+\frac{C_{10}}{3}-C_{5}-\frac{C_{6}}{3}-C_{7}-\frac{C_{8}}{3}\right) F_{a K}^{L L} \\
& \left.\left.+\left(C_{4}+C_{10}\right) M_{a K}^{L L}+\left(C_{6}+C_{8}\right) M_{a K}^{S P}\right]\right\}, \\
& \mathcal{A}\left(B^{0} \rightarrow K^{-}\left(K^{*+} \rightarrow\right) K \pi\right)=\frac{G_{F}}{\sqrt{2}}\left\{V_{u b}^{*} V_{u d}\left[\left(C_{1}+\frac{C_{2}}{3}\right) F_{a K^{*}}^{L L}+C_{2} M_{a K^{*}}^{L L}\right]\right. \\
& -V_{t b}^{*} V_{t d}\left[\left(C_{3}+\frac{C_{4}}{3}+C_{9}+\frac{C_{10}}{3}-C_{5}-\frac{C_{6}}{3}-C_{7}-\frac{C_{8}}{3}\right) F_{a K^{*}}^{L L}+\left(C_{4}+C_{10}\right) M_{a K^{*}}^{L L}\right. \\
& +\left(C_{6}+C_{8}\right) M_{a K^{*}}^{S P}+\left(C_{3}+\frac{C_{4}}{3}-\frac{C_{9}}{2}-\frac{C_{10}}{6}-C_{5}-\frac{C_{6}}{3}+\frac{C_{7}}{2}+\frac{C_{8}}{6}\right) F_{a K}^{L L} \\
& \left.\left.+\left(C_{4}-\frac{C_{10}}{2}\right) M_{a K}^{L L}+\left(C_{6}-\frac{C_{8}}{2}\right) M_{a K}^{S P}\right]\right\},
\end{aligned}
$$




$$
\begin{aligned}
\mathcal{A}\left(B_{s}^{0} \rightarrow K^{+}\left(K^{*-} \rightarrow\right) K \pi\right)= & \frac{G_{F}}{\sqrt{2}}\left\{V_{u b}^{*} V_{u s}\left[\left(\frac{C_{1}}{3}+C_{2}\right) F_{e K^{*}}^{L L}+C_{1} M_{e K^{*}}^{L L}+\left(C_{1}+\frac{C_{2}}{3}\right) F_{a K}^{L L}+C_{2} M_{a K}^{L L}\right]\right. \\
& -V_{t b}^{*} V_{t s}\left[\left(\frac{C_{3}}{3}+C_{4}+\frac{C_{9}}{3}+C_{10}\right) F_{e K^{*}}^{L L}+\left(\frac{C_{5}}{3}+C_{6}+\frac{C_{7}}{3}+C_{8}\right) F_{e K^{*}}^{S P}\right. \\
& +\left(C_{3}+C_{9}\right) M_{e K^{*}}^{L L}+\left(C_{5}+C_{7}\right) M_{e K^{*}}^{L R}+\left(\frac{4}{3}\left(C_{3}+C_{4}-\frac{C_{9}}{2}-\frac{C_{10}}{2}\right)\right. \\
& \left.-C_{5}-\frac{C_{6}}{3}+\frac{C_{7}}{2}+\frac{C_{8}}{6}\right) F_{a K^{*}}^{L L}+\left(\frac{C_{5}}{3}+C_{6}-\frac{C_{7}}{6}-\frac{C_{8}}{2}\right) F_{a K^{*}}^{S P} \\
& +\left(C_{3}+C_{4}-\frac{C_{9}}{2}-\frac{C_{10}}{2}\right) M_{a K^{*}}^{L L}+\left(C_{5}-\frac{C_{7}}{2}\right) M_{a K^{*}}^{L R}+\left(C_{6}-\frac{C_{8}}{2}\right) M_{a K^{*}}^{S P} \\
& +\left(C_{3}+\frac{C_{4}}{3}+C_{9}+\frac{C_{10}}{3}-C_{5}-\frac{C_{6}}{3}-C_{7}-\frac{C_{8}}{3}\right) F_{a K}^{L L}+\left(C_{4}+C_{10}\right) M_{a K}^{L L} \\
& \left.\left.+\left(C_{6}+C_{8}\right) M_{a K}^{S P}\right]\right\},
\end{aligned}
$$

$$
\begin{aligned}
\mathcal{A}\left(B_{s}^{0} \rightarrow K^{-}\left(K^{*+} \rightarrow\right) K \pi\right)= & \frac{G_{F}}{\sqrt{2}}\left\{V_{u b}^{*} V_{u s}\left[\left(C_{1}+\frac{C_{2}}{3}\right) F_{a K^{*}}^{L L}+C_{2} M_{a K^{*}}^{L L}+\left(\frac{C_{1}}{3}+C_{2}\right) F_{e K}^{L L}+C_{1} M_{e K}^{L L}\right]\right. \\
& -V_{t b}^{*} V_{t s}\left[\left(C_{3}+\frac{C_{4}}{3}+C_{9}+\frac{C_{10}}{3}-C_{5}-\frac{C_{6}}{3}-C_{7}-\frac{C_{8}}{3}\right) F_{a K^{*}}^{L L}+\left(C_{4}+C_{10}\right) M_{a K^{*}}^{L L}\right. \\
& +\left(C_{6}+C_{8}\right) M_{a K^{*}}^{S P}+\left(\frac{C_{3}}{3}+C_{4}+\frac{C_{9}}{3}+C_{10}\right) F_{e K}^{L L}+\left(C_{3}+C_{9}\right) M_{e K}^{L L}+\left(C_{5}+C_{7}\right) M_{e K}^{L R} \\
& +\left(\frac{4}{3}\left(C_{3}+C_{4}-\frac{C_{9}}{2}-\frac{C_{10}}{2}\right)-C_{5}-\frac{C_{6}}{3}+\frac{C_{7}}{2}+\frac{C_{8}}{6}\right) F_{a K}^{L L} \\
& +\left(\frac{C_{5}}{3}+C_{6}-\frac{C_{7}}{6}-\frac{C_{8}}{2}\right) F_{a K}^{S P}+\left(C_{3}+C_{4}-\frac{C_{9}}{2}-\frac{C_{10}}{2}\right) M_{a K}^{L L} \\
& \left.\left.+\left(C_{5}-\frac{C_{7}}{2}\right) M_{a K}^{L R}+\left(C_{6}-\frac{C_{8}}{2}\right) M_{a K}^{S P}\right]\right\}
\end{aligned}
$$

$$
\begin{aligned}
\mathcal{A}\left(B^{+} \rightarrow \bar{K}^{0}\left(K^{*+} \rightarrow\right) K \pi\right)= & \frac{G_{F}}{\sqrt{2}}\left\{V_{u b}^{*} V_{u d}\left[\left(\frac{C_{1}}{3}+C_{2}\right) F_{a K^{*}}^{L L}+C_{1} M_{a K^{*}}^{L L}\right]-V_{t b}^{*} V_{t d}\left[\left(\frac{C_{3}}{3}+C_{4}-\frac{C_{9}}{6}-\frac{C_{10}}{2}\right) F_{e K^{*}}^{L L}\right.\right. \\
& +\left(\frac{C_{5}}{3}+C_{6}-\frac{C_{7}}{6}-\frac{C_{8}}{2}\right) F_{e K^{*}}^{S P}+\left(C_{3}-\frac{C_{9}}{2}\right) M_{e K^{*}}^{L L}+\left(C_{5}-\frac{C_{7}}{2}\right) M_{e K^{*}}^{L R} \\
& +\left(\frac{C_{3}}{3}+C_{4}+\frac{C_{9}}{3}+C_{10}\right) F_{a K^{*}}^{L L}+\left(\frac{C_{5}}{3}+C_{6}+\frac{C_{7}}{3}+C_{8}\right) F_{a K^{*}}^{S P} \\
& \left.\left.+\left(C_{3}+C_{9}\right) M_{a K^{*}}^{L L}+\left(C_{5}+C_{7}\right) M_{a K^{*}}^{L R}\right]\right\}
\end{aligned}
$$$$
\mathcal{A}\left(B^{0} \rightarrow K^{0}\left(\bar{K}^{* 0} \rightarrow\right) K \pi\right)=-\frac{G_{F}}{\sqrt{2}}\left\{V _ { t b } ^ { * } V _ { t d } \left[\left(C_{3}+\frac{C_{4}}{3}-\frac{C_{9}}{2}-\frac{C_{10}}{6}-C_{5}-\frac{C_{6}}{3}+\frac{C_{7}}{2}+\frac{C_{8}}{6}\right) F_{a K^{*}}^{L L}+\left(C_{4}-\frac{C_{10}}{2}\right) M_{a K^{*}}^{L L}\right.\right.
$$$$
+\left(C_{6}-\frac{C_{8}}{2}\right)\left(M_{a K^{*}}^{S P}+M_{a K}^{S P}\right)+\left(\frac{C_{3}}{3}+C_{4}-\frac{C_{9}}{6}-\frac{C_{10}}{2}\right) F_{e K}^{L L}+\left(C_{3}-\frac{C_{9}}{2}\right) M_{e K}^{L L}
$$$$
+\left(C_{5}-\frac{C_{7}}{2}\right)\left(M_{e K}^{L R}+M_{a K}^{L R}\right)+\left(\frac{C_{5}}{3}+C_{6}-\frac{C_{7}}{6}-\frac{C_{8}}{2}\right) F_{a K}^{S P}
$$$$
\left.\left.+\left(\frac{4}{3}\left(C_{3}+C_{4}-\frac{C_{9}}{2}-\frac{C_{10}}{2}\right)-C_{5}-\frac{C_{6}}{3}+\frac{C_{7}}{2}+\frac{C_{8}}{6}\right) F_{a K}^{L L}+\left(C_{3}+C_{4}-\frac{C_{9}}{2}-\frac{C_{10}}{2}\right) M_{a K}^{L L}\right]\right\},
$$ 


$$
\begin{aligned}
\mathcal{A}\left(B^{0} \rightarrow \bar{K}^{0}\left(K^{* 0} \rightarrow\right) K \pi\right)= & -\frac{G_{F}}{\sqrt{2}}\left\{V _ { t b } ^ { * } V _ { t d } \left[\left(\frac{C_{3}}{3}+C_{4}-\frac{C_{9}}{6}-\frac{C_{10}}{2}\right) F_{e K^{*}}^{L L}+\left(\frac{C_{5}}{3}+C_{6}-\frac{C_{7}}{6}-\frac{C_{8}}{2}\right)\left(F_{e K^{*}}^{S P}+F_{a K^{*}}^{S P}\right)\right.\right. \\
& +\left(C_{3}-\frac{C_{9}}{2}\right) M_{e K^{*}}^{L L}+\left(\frac{4}{3}\left(C_{3}+C_{4}-\frac{C_{9}}{2}-\frac{C_{10}}{2}\right)-C_{5}-\frac{C_{6}}{3}+\frac{C_{7}}{2}+\frac{C_{8}}{6}\right) F_{a K^{*}}^{L L} \\
& +\left(C_{3}+C_{4}-\frac{C_{9}}{2}-\frac{C_{10}}{2}\right) M_{a K^{*}}^{L L}+\left(C_{5}-\frac{C_{7}}{2}\right)\left(M_{e K^{*}}^{L R}+M_{a K^{*}}^{L R}\right) \\
& +\left(C_{6}-\frac{C_{8}}{2}\right)\left(M_{a K^{*}}^{S P}+M_{a K}^{S P}\right)+\left(C_{3}+\frac{C_{4}}{3}-\frac{C_{9}}{2}-\frac{C_{10}}{6}-C_{5}-\frac{C_{6}}{3}+\frac{C_{7}}{2}+\frac{C_{8}}{6}\right) F_{a K}^{L L} \\
& \left.\left.+\left(C_{4}-\frac{C_{10}}{2}\right) M_{a K}^{L L}\right]\right\},
\end{aligned}
$$$$
\mathcal{A}\left(B_{s}^{0} \rightarrow K^{0}\left(\bar{K}^{* 0} \rightarrow\right) K \pi\right)=-\frac{G_{F}}{\sqrt{2}}\left\{V _ { t b } ^ { * } V _ { t s } \left[\left(\frac{C_{3}}{3}+C_{4}-\frac{C_{9}}{6}-\frac{C_{10}}{2}\right) F_{e K^{*}}^{L L}+\left(\frac{C_{5}}{3}+C_{6}-\frac{C_{7}}{6}-\frac{C_{8}}{2}\right)\left(F_{e K^{*}}^{S P}+F_{a K^{*}}^{S P}\right)\right.\right.
$$$$
+\left(C_{3}-\frac{C_{9}}{2}\right) M_{e K^{*}}^{L L}+\left(\frac{4}{3}\left(C_{3}+C_{4}-\frac{C_{9}}{2}-\frac{C_{10}}{2}\right)-C_{5}-\frac{C_{6}}{3}+\frac{C_{7}}{2}+\frac{C_{8}}{6}\right) F_{a K^{*}}^{L L}
$$$$
+\left(C_{3}+C_{4}-\frac{C_{9}}{2}-\frac{C_{10}}{2}\right) M_{a K^{*}}^{L L}+\left(C_{5}-\frac{C_{7}}{2}\right)\left(M_{e K^{*}}^{L R}+M_{a K^{*}}^{L R}\right)+\left(C_{6}-\frac{C_{8}}{2}\right)\left(M_{a K^{*}}^{S P}\right.
$$$$
\left.\left.\left.+M_{a K}^{S P}\right)+\left(C_{3}+\frac{C_{4}}{3}-\frac{C_{9}}{2}-\frac{C_{10}}{6}-C_{5}-\frac{C_{6}}{3}+\frac{C_{7}}{2}+\frac{C_{8}}{6}\right) F_{a K}^{L L}+\left(C_{4}-\frac{C_{10}}{2}\right) M_{a K}^{L L}\right]\right\},
$$

$$
\begin{aligned}
\mathcal{A}\left(B_{s}^{0} \rightarrow \bar{K}^{0}\left(K^{* 0} \rightarrow\right) K \pi\right)= & -\frac{G_{F}}{\sqrt{2}}\left\{V _ { t b } ^ { * } V _ { t s } \left[\left(C_{3}+\frac{C_{4}}{3}-\frac{C_{9}}{2}-\frac{C_{10}}{6}-C_{5}-\frac{C_{6}}{3}+\frac{C_{7}}{2}+\frac{C_{8}}{6}\right) F_{a K^{*}}^{L L}\right.\right. \\
& +\left(C_{4}-\frac{C_{10}}{2}\right) M_{a K^{*}}^{L L}+\left(C_{6}-\frac{C_{8}}{2}\right)\left(M_{a K^{*}}^{S P}+M_{a K}^{S P}\right) \\
& +\left(\frac{C_{3}}{3}+C_{4}-\frac{C_{9}}{6}-\frac{C_{10}}{2}\right) F_{e K}^{L L}+\left(C_{3}-\frac{C_{9}}{2}\right) M_{e K}^{L L}+\left(C_{5}-\frac{C_{7}}{2}\right)\left(M_{e K}^{L R}+M_{a K}^{L R}\right) \\
& +\left(\frac{C_{5}}{3}+C_{6}-\frac{C_{7}}{6}-\frac{C_{8}}{2}\right) F_{a K}^{S P}+\left(\frac{4}{3}\left(C_{3}+C_{4}-\frac{C_{9}}{2}-\frac{C_{10}}{2}\right)-C_{5}-\frac{C_{6}}{3}+\frac{C_{7}}{2}+\frac{C_{8}}{6}\right) F_{a K}^{L L} \\
& \left.\left.+\left(C_{3}+C_{4}-\frac{C_{9}}{2}-\frac{C_{10}}{2}\right) M_{a K}^{L L}\right]\right\} .
\end{aligned}
$$

(4) $B_{(s)} \rightarrow \pi\left(K^{*} \rightarrow\right) K \pi$

$$
\begin{aligned}
\mathcal{A}\left(B^{+} \rightarrow \pi^{+}\left(K^{* 0} \rightarrow\right) K \pi\right)= & \frac{G_{F}}{\sqrt{2}}\left\{V_{u b}^{*} V_{u s}\left[\left(\frac{C_{1}}{3}+C_{2}\right) F_{a \pi}^{L L}+C_{1} M_{a \pi}^{L L}\right]-V_{t b}^{*} V_{t s}\left[\left(\frac{C_{3}}{3}+C_{4}-\frac{C_{9}}{6}-\frac{C_{10}}{2}\right) F_{e \pi}^{L L}\right.\right. \\
& +\left(C_{3}-\frac{C_{9}}{2}\right) M_{e \pi}^{L L}+\left(C_{5}-\frac{C_{7}}{2}\right) M_{e \pi}^{L R}+\left(\frac{C_{3}}{3}+C_{4}+\frac{C_{9}}{3}+C_{10}\right) F_{a \pi}^{L L} \\
& \left.\left.+\left(\frac{C_{5}}{3}+C_{6}+\frac{C_{7}}{3}+C_{8}\right) F_{a \pi}^{S P}+\left(C_{3}+C_{9}\right) M_{a \pi}^{L L}+\left(C_{5}+C_{7}\right) M_{a \pi}^{L R}\right]\right\},
\end{aligned}
$$




$$
\begin{aligned}
\mathcal{A}\left(B^{0} \rightarrow \pi^{-}\left(K^{*+} \rightarrow\right) K \pi\right)= & \frac{G_{F}}{\sqrt{2}}\left\{V_{u b}^{*} V_{u s}\left[\left(\frac{C_{1}}{3}+C_{2}\right) F_{e \pi}^{L L}+C_{1} M_{e \pi}^{L L}\right]-V_{t b}^{*} V_{t s}\left[\left(\frac{C_{3}}{3}+C_{4}+\frac{C_{9}}{3}+C_{10}\right) F_{e \pi}^{L L}\right.\right. \\
+ & \left(C_{3}+C_{9}\right) M_{e \pi}^{L L}+\left(C_{5}+C_{7}\right) M_{e \pi}^{L R}+\left(\frac{C_{3}}{3}+C_{4}-\frac{C_{9}}{6}-\frac{C_{10}}{2}\right) F_{a \pi}^{L L} \\
+ & \left.\left.\left(\frac{C_{5}}{3}+C_{6}-\frac{C_{7}}{6}-\frac{C_{8}}{2}\right) F_{a \pi}^{S P}+\left(C_{3}-\frac{C_{9}}{2}\right) M_{a \pi}^{L L}+\left(C_{5}-\frac{C_{7}}{2}\right) M_{a \pi}^{L R}\right]\right\}, \quad(\mathrm{A} 26 \\
\mathcal{A}\left(B_{s}^{0} \rightarrow \pi^{+}\left(K^{*-} \rightarrow\right) K \pi\right)= & \frac{G_{F}}{\sqrt{2}}\left\{V_{u b}^{*} V_{u d}\left[\left(\frac{C_{1}}{3}+C_{2}\right) F_{e K^{*}}^{L L}+C_{1} M_{e K^{*}}^{L L}\right]\right. \\
& -V_{t b}^{*} V_{t d}\left[\left(\frac{C_{3}}{3}+C_{4}+\frac{C_{9}}{3}+C_{10}\right) F_{e K^{*}}^{L L}+\left(\frac{C_{5}}{3}+C_{6}+\frac{C_{7}}{3}+C_{8}\right) F_{e K^{*}}^{S P}\right. \\
& +\left(C_{3}+C_{9}\right) M_{e K^{*}}^{L L}+\left(C_{5}+C_{7}\right) M_{e K^{*}}^{L R}+\left(\frac{C_{3}}{3}+C_{4}-\frac{C_{9}}{6}-\frac{C_{10}}{2}\right) F_{a K^{*}}^{L L} \\
& \left.\left.+\left(\frac{C_{5}}{3}+C_{6}-\frac{C_{7}}{6}-\frac{C_{8}}{2}\right) F_{a K^{*}}^{S P}+\left(C_{3}-\frac{C_{9}}{2}\right) M_{a K^{*}}^{L L}+\left(C_{5}-\frac{C_{7}}{2}\right) M_{a K^{*}}^{L R}\right]\right\}, \quad(\mathrm{A} 27
\end{aligned}
$$

$$
\begin{aligned}
\mathcal{A}\left(B^{+} \rightarrow \pi^{0}\left(K^{*+} \rightarrow\right) K \pi\right)= & \frac{G_{F}}{2}\left\{V _ { u b } ^ { * } V _ { u s } \left[\left(C_{1}+\frac{C_{2}}{3}\right) F_{e K^{*}}^{L L}+\left(\frac{C_{1}}{3}+C_{2}\right)\left(F_{e \pi}^{L L}+F_{a \pi}^{L L}\right)\right.\right. \\
& \left.+C_{1}\left(M_{e \pi}^{L L}+M_{a \pi}^{L L}\right)+C_{2} M_{e K^{*}}^{L L}\right]-V_{t b}^{*} V_{t s}\left[\left(\frac{3 C_{9}}{2}+\frac{C_{10}}{2}-\frac{3 C_{7}}{2}-\frac{C_{8}}{2}\right) F_{e K^{*}}^{L L}+\frac{3 C_{10}}{2} M_{e K^{*}}^{L L}\right. \\
& +\frac{3 C_{8}}{2} M_{e K^{*}}^{S P}+\left(\frac{C_{3}}{3}+C_{4}+\frac{C_{9}}{3}+C_{10}\right)\left(F_{e \pi}^{L L}+F_{a \pi}^{L L}\right)+\left(\frac{C_{5}}{3}+C_{6}+\frac{C_{7}}{3}+C_{8}\right) F_{a \pi}^{S P} \\
& \left.\left.+\left(C_{3}+C_{9}\right)\left(M_{e \pi}^{L L}+M_{a \pi}^{L L}\right)+\left(C_{5}+C_{7}\right)\left(M_{e \pi}^{L R}+M_{a \pi}^{L R}\right)\right]\right\}
\end{aligned}
$$

$$
\begin{aligned}
\mathcal{A}\left(B^{0} \rightarrow \pi^{0}\left(K^{* 0} \rightarrow\right) K \pi\right)= & \frac{G_{F}}{2}\left\{V_{u b}^{*} V_{u s}\left[\left(C_{1}+\frac{C_{2}}{3}\right) F_{e K^{*}}^{L L}+C_{2} M_{e K^{*}}^{L L}\right]-V_{t b}^{*} V_{t s}\left[\left(\frac{3 C_{9}}{2}+\frac{C_{10}}{2}-\frac{3 C_{7}}{2}-\frac{C_{8}}{2}\right) F_{e K^{*}}^{L L}\right.\right. \\
& +\frac{3 C_{10}}{2} M_{e K^{*}}^{L L}+\frac{3 C_{8}}{2} M_{e K^{*}}^{S P}-\left(\frac{C_{3}}{3}+C_{4}-\frac{C_{9}}{6}-\frac{C_{10}}{2}\right)\left(F_{e \pi}^{L L}+F_{a \pi}^{L L}\right) \\
& \left.\left.-\left(\frac{C_{5}}{3}+C_{6}-\frac{C_{7}}{6}-\frac{C_{8}}{2}\right) F_{a \pi}^{S P}-\left(C_{3}-\frac{C_{9}}{2}\right)\left(M_{e \pi}^{L L}+M_{a \pi}^{L L}\right)-\left(C_{5}-\frac{C_{7}}{2}\right)\left(M_{e \pi}^{L R}+M_{a \pi}^{L R}\right)\right]\right\},
\end{aligned}
$$

$$
\begin{aligned}
\mathcal{A}\left(B_{s}^{0} \rightarrow \pi^{0}\left(\bar{K}^{* 0} \rightarrow\right) K \pi\right)= & \frac{G_{F}}{2}\left\{V_{u b}^{*} V_{u d}\left[\left(C_{1}+\frac{C_{2}}{3}\right) F_{e K^{*}}^{L L}+C_{2} M_{e K^{*}}^{L L}\right]\right. \\
& -V_{t b}^{*} V_{t d}\left[\left(-\frac{C_{3}}{3}-C_{4}+\frac{5 C_{9}}{3}+C_{10}-\frac{3 C_{7}}{2}-\frac{C_{8}}{2}\right) F_{e K^{*}}^{L L}-\left(\frac{C_{5}}{3}+C_{6}-\frac{C_{7}}{6}-\frac{C_{8}}{2}\right) F_{e K^{*}}^{S P}\right. \\
& +\left(-C_{3}+\frac{C_{9}}{2}+\frac{3 C_{10}}{2}\right) M_{e K^{*}}^{L L}-\left(C_{5}-\frac{C_{7}}{2}\right) M_{e K^{*}}^{L R}+\frac{3 C_{8}}{2} M_{e K^{*}}^{S P} \\
& -\left(\frac{C_{3}}{3}+C_{4}-\frac{C_{9}}{6}-\frac{C_{10}}{2}\right) F_{a K^{*}}^{L L}+\left(\frac{C_{5}}{3}+C_{6}-\frac{C_{7}}{6}-\frac{C_{8}}{2}\right) F_{a K^{*}}^{S P} \\
& \left.\left.-\left(C_{3}-\frac{C_{9}}{2}\right) M_{a K^{*}}^{L L}-\left(C_{5}-\frac{C_{7}}{2}\right) M_{a K^{*}}^{L R}\right]\right\} .
\end{aligned}
$$


(5) $B_{(s)} \rightarrow K(\phi \rightarrow) K K$

$$
\begin{aligned}
& \mathcal{A}\left(B^{+} \rightarrow K^{+}(\phi \rightarrow) K K\right)=\frac{G_{F}}{\sqrt{2}}\left\{V_{u b}^{*} V_{u s}\left[\left(\frac{C_{1}}{3}+C_{2}\right) F_{a K}^{L L}+C_{1} M_{a K}^{L L}\right]-\frac{G_{F}}{\sqrt{2}} V_{t b}^{*} V_{t s}\left[\left(\frac{4}{3}\left(C_{3}+C_{4}\right)\right.\right.\right. \\
& \left.+C_{5}+\frac{C_{6}}{3}-\frac{C_{7}}{2}-\frac{C_{8}}{6}-\frac{2}{3}\left(C_{9}+C_{10}\right)\right) F_{e K}^{L L}+\left(C_{3}+C_{4}-\frac{1}{2}\left(C_{9}+C_{10}\right)\right) M_{e K}^{L L} \\
& +\left(C_{5}-\frac{C_{7}}{2}\right) M_{e K}^{L R}+\left(C_{6}-\frac{C_{8}}{2}\right) M_{e K}^{S P}+\left(\frac{C_{3}}{3}+C_{4}+\frac{C_{9}}{3}+C_{10}\right) F_{a K}^{L L} \\
& \left.\left.+\left(\frac{C_{5}}{3}+C_{6}+\frac{C_{7}}{3}+C_{8}\right) F_{a K}^{S P}+\left(C_{3}+C_{9}\right) M_{a K}^{L L}+\left(C_{5}+C_{7}\right) M_{a K}^{L R}\right]\right\}, \\
& \mathcal{A}\left(B^{0} \rightarrow K^{0}(\phi \rightarrow) K K\right)=-\frac{G_{F}}{\sqrt{2}}\left\{V _ { t b } ^ { * } V _ { t s } \left[\left(\frac{4}{3} C_{3}+\frac{4}{3} C_{4}+C_{5}+\frac{C_{6}}{3}-\frac{C_{7}}{2}-\frac{C_{8}}{6}-\frac{2}{3} C_{9}-\frac{2}{3} C_{10}\right) F_{e K}^{L L}\right.\right. \\
& +\left(C_{3}+C_{4}-\frac{C_{9}}{2}-\frac{C_{10}}{2}\right) M_{e K}^{L L}+\left(C_{5}-\frac{C_{7}}{2}\right) M_{e K}^{L R} \\
& +\left(C_{6}-\frac{C_{8}}{2}\right) M_{e K}^{S P}+\left(\frac{C_{3}}{3}+C_{4}-\frac{C_{9}}{6}-\frac{C_{10}}{2}\right) F_{a K}^{L L}+\left(\frac{C_{5}}{3}+C_{6}-\frac{C_{7}}{6}-\frac{C_{8}}{2}\right) F_{a K}^{S P} \\
& \left.\left.+\left(C_{3}-\frac{C_{9}}{2}\right) M_{a K}^{L L}+\left(C_{5}-\frac{C_{7}}{2}\right) M_{a K}^{L R}\right]\right\}, \\
& A\left(B_{s}^{0} \rightarrow \bar{K}^{0}(\phi \rightarrow) K K\right)=-\frac{G_{F}}{\sqrt{2}}\left\{V _ { t b } ^ { * } V _ { t d } \left[\left(C_{3}+\frac{C_{4}}{3}+C_{5}+\frac{C_{6}}{3}-\frac{C_{7}}{2}-\frac{C_{8}}{6}-\frac{C_{9}}{2}-\frac{C_{10}}{6}\right) F_{e K}^{L L}\right.\right. \\
& +\left(\frac{C_{3}}{3}+C_{4}-\frac{C_{9}}{6}-\frac{C_{10}}{2}\right)\left(F_{e \phi}^{L L}+F_{a \phi}^{L L}\right)+\left(\frac{C_{5}}{3}+C_{6}-\frac{C_{7}}{6}-\frac{C_{8}}{2}\right)\left(F_{e \phi}^{S P}+F_{a \phi}^{S P}\right) \\
& +\left(C_{3}-\frac{C_{9}}{2}\right)\left(M_{e \phi}^{L L}+M_{a \phi}^{L L}\right)+\left(C_{5}-\frac{C_{7}}{2}\right)\left(M_{e \phi}^{L R}+M_{a \phi}^{L R}\right)+\left(C_{4}-\frac{C_{10}}{2}\right) M_{e K}^{L L} \\
& \left.\left.+\left(C_{6}-\frac{C_{8}}{2}\right) M_{e K}^{S P}\right]\right\} \text {. }
\end{aligned}
$$

(6) $B_{(s)} \rightarrow \pi(\phi \rightarrow) K K$

$$
\begin{aligned}
& \mathcal{A}\left(B^{+} \rightarrow \pi^{+}(\phi \rightarrow) K K\right)=-\frac{G_{F}}{\sqrt{2}}\left\{V _ { t b } ^ { * } V _ { t d } \left[\left(C_{3}+\frac{C_{4}}{3}+C_{5}+\frac{C_{6}}{3}-\frac{C_{7}}{2}-\frac{C_{8}}{6}-\frac{C_{9}}{2}-\frac{C_{10}}{6}\right) F_{e \pi}^{L L}\right.\right. \\
& \left.\left.+\left(C_{4}-\frac{C_{10}}{2}\right) M_{e \pi}^{L L}+\left(C_{6}-\frac{C_{8}}{2}\right) M_{e \pi}^{S P}\right]\right\} \text {, } \\
& \sqrt{2} \mathcal{A}\left(B^{0} \rightarrow \pi^{0}(\phi \rightarrow) K K\right)=-\frac{G_{F}}{\sqrt{2}}\left\{V _ { t b } ^ { * } V _ { t d } \left[\left(-C_{3}-\frac{C_{4}}{3}-C_{5}-\frac{C_{6}}{3}+\frac{C_{7}}{2}+\frac{C_{8}}{6}+\frac{C_{9}}{2}+\frac{C_{10}}{6}\right) F_{e \pi}^{L L}\right.\right. \\
& \left.\left.+\left(-C_{4}+\frac{C_{10}}{2}\right) M_{e \pi}^{L L}+\left(-C_{6}+\frac{C_{8}}{2}\right) M_{e \pi}^{S P}\right]\right\} \text {, } \\
& \sqrt{2} A\left(B_{s}^{0} \rightarrow \pi^{0}(\phi \rightarrow) K K\right)=\frac{G_{F}}{\sqrt{2}}\left\{V_{u b}^{*} V_{u s}\left[\left(C_{1}+\frac{C_{2}}{3}\right) F_{e \phi}^{L L}+C_{2} M_{e \phi}^{L L}\right]\right. \\
& \left.-\frac{G_{F}}{\sqrt{2}} V_{t b}^{*} V_{t s}\left[\left(-\frac{3}{2} C_{7}-\frac{C_{8}}{2}+\frac{3}{2} C_{9}+\frac{C_{10}}{2}\right) F_{e \phi}^{L L}+\frac{3}{2} C_{8} M_{e \phi}^{S P}+\frac{3}{2} C_{10} M_{e \phi}^{L L}\right]\right\} \text {. }
\end{aligned}
$$

The explicit PQCD factorization formulas for the functions $F$ and $M$ appearing in the above decay amplitudes are given by 


$$
\begin{aligned}
& F_{e V}^{L L}=8 \pi C_{F} f_{P} m_{B}^{4} \int_{0}^{1} d x_{B} d z \int_{0}^{\infty} b_{B} d b_{B} b d b \phi_{B}\left(x_{B}, b_{B}\right) \\
& \times\left\{\left[\left(\left(f_{-} g_{-}-f_{+} g_{+}\left(1+f_{+} z\right)\right) \sqrt{\eta} \phi_{0}(z)-\left(g_{-}+g_{+}\left(1-2 f_{+} z\right)\right) \eta \phi_{s}(z)\right.\right.\right. \\
& \left.\left.+f_{-} f_{+}\left(g_{-}+g_{+}\left(-1+2 f_{+} z\right)\right) \phi_{t}(z)\right) / \sqrt{\eta}\right] E_{e}\left(t_{a}^{V}\right) h_{a}^{V}\left(\alpha_{e}^{V}, \beta_{a}^{V}, b_{B}, b\right) S_{t}(z) \\
& +\left[f_{+}\left(f_{-}\left(g_{-}-g_{+}\right)-g_{-} x_{B}\right) \phi_{0}(z)-2\left(\left(f_{-}-x_{B}\right) g_{-}+f_{+} g_{+}\right) \sqrt{\eta} \phi_{s}(z)\right] \\
& \left.\times E_{e}\left(t_{b}^{V}\right) h_{b}^{V}\left(\alpha_{e}^{V}, \beta_{b}^{V}, b_{B}, b\right) S_{t}\left(\left|x_{B}-f_{-}\right|\right)\right\}, \\
& F_{e V}^{L R}=-F_{e V}^{L L} \\
& F_{e V}^{S P}=16 \pi C_{F} f_{P} m_{B}^{4} r_{03} \int_{0}^{1} d x_{B} d z \int_{0}^{\infty} b_{B} d b_{B} b d b \phi_{B}\left(x_{B}, b_{B}\right) \\
& \times\left\{\left[\left(\left(f_{+}+f_{-}\left(-1+2 f_{+} z\right)\right) \sqrt{\eta} \phi_{0}(z)+\left(2+f_{+} z\right) \eta \phi_{s}(z)-f_{-} f_{+}^{2} z \phi_{t}(z)\right) / \sqrt{\eta}\right]\right. \\
& \times E_{e}\left(t_{a}^{V}\right) h_{a}^{V}\left(\alpha_{e}^{V}, \beta_{a}^{V}, b_{B}, b\right) S_{t}(z) \\
& \left.+\left[f_{+} x_{B} \phi_{0}(z)+2\left(f_{-}+f_{+}-x_{B}\right) \sqrt{\eta} \phi_{s}(z)\right] E_{e}\left(t_{b}^{V}\right) h_{b}^{V}\left(\alpha_{e}^{V}, \beta_{b}^{V}, b_{B}, b\right) S_{t}\left(\left|x_{B}-f_{-}\right|\right)\right\}, \\
& M_{e V}^{L L}=32 \pi C_{F} m_{B}^{4} / \sqrt{6} / \sqrt{\eta} \int_{0}^{1} d x_{B} d z d x_{3} \int_{0}^{\infty} b_{B} d b_{B} b_{3} d b_{3} \phi_{B}\left(x_{B}, b_{B}\right) \phi_{P}^{A}\left(x_{3}\right) \\
& \times\left\{\left[\left(g_{-}+g_{+}\right)\left(f_{+}\left(g_{+}\left(-1+x_{3}\right)+x_{B}\right)+f_{-}\left(g_{-}+f_{+} z\right)\right) \sqrt{\eta} \phi_{0}(z)+\left(-g_{-}\left(g_{+}\left(x_{3}-2\right)+x_{B}\right)\right.\right.\right. \\
& \left.\left.+f_{+} g_{+} z\right) \eta \phi_{s}(z)-f_{-} f_{+}\left(g_{-}\left(g_{+} x_{3}+x_{B}\right)+f_{+} g_{+} z\right) \phi_{t}(z)\right] E_{n}\left(t_{c}^{V}\right) h_{c}^{V}\left(\alpha_{e}^{V}, \beta_{c}^{V}, b_{B}, b_{3}\right) \\
& -\left[\left(f_{-} g_{-}-f_{+} g_{+}\right)\left(g_{+} x_{3}-x_{B}+f_{+} z\right) \sqrt{\eta} \phi_{0}(z)+\left(g_{-} g_{+} x_{3}-g_{-} x_{B}+f_{+} g_{+} z\right) \eta \phi_{s}(z)\right. \\
& \left.\left.+f_{-} f_{+}\left(g_{-}\left(-g_{+} x_{3}+x_{B}\right)+f_{+} g_{+} z\right) \phi_{t}(z)\right] E_{n}\left(t_{d}^{V}\right) h_{d}^{V}\left(\alpha_{e}^{V}, \beta_{d}^{V}, b_{B}, b_{3}\right)\right\} \text {, } \\
& M_{e V}^{L R}=-32 \pi C_{F} m_{B}^{4} r_{03} / \sqrt{6} / \sqrt{\eta} \int_{0}^{1} d x_{B} d z d x_{3} \int_{0}^{\infty} b_{B} d b_{B} b_{3} d b_{3} \phi_{B}\left(x_{B}, b_{B}\right) \\
& \times\left\{\left[\sqrt{\eta} \phi_{0}(z)\left(f_{-}\left(g_{-}+f_{+} z\right)\left(\phi_{P}^{P}\left(x_{3}\right)-\phi_{P}^{T}\left(x_{3}\right)\right)+f_{+}\left(g_{+}\left(x_{3}-1\right)+x_{B}\right)\left(\phi_{P}^{P}\left(x_{3}\right)+\phi_{P}^{T}\left(x_{3}\right)\right)\right)\right.\right. \\
& -\left(\eta \phi_{s}(z)+f_{+} f_{-} \phi_{t}(z)\right)\left(f_{+} z+g_{-}\right)\left(\phi_{P}^{P}\left(x_{3}\right)-\phi_{P}^{T}\left(x_{3}\right)\right)+\left(\eta \phi_{s}(z)-f_{+} f_{-} \phi_{t}(z)\right)\left(x_{B}\right. \\
& \left.\left.+g_{+}\left(x_{3}-1\right)\right)\left(\phi_{P}^{P}\left(x_{3}\right)+\phi_{P}^{T}\left(x_{3}\right)\right)\right] E_{n}\left(t_{c}^{V}\right) h_{c}^{V}\left(\alpha_{e}^{V}, \beta_{c}^{V}, b_{B}, b_{3}\right) \\
& +\left[f_{+} \sqrt{\eta} \phi_{0}(z)\left(\left(g_{+} x_{3}-x_{B}\right)\left(\phi_{P}^{P}\left(x_{3}\right)-\phi_{P}^{T}\left(x_{3}\right)\right)-f_{-} z\left(\phi_{P}^{P}\left(x_{3}\right)+\phi_{P}^{T}\left(x_{3}\right)\right)\right)\right. \\
& +\left(g_{+} x_{3}-x_{B}\right)\left(\phi_{P}^{P}\left(x_{3}\right)-\phi_{P}^{T}\left(x_{3}\right)\right)\left(\eta \phi_{s}(z)-f_{+} f_{-} \phi_{t}(z)\right) \\
& \left.\left.+f_{+} z\left(\phi_{P}^{P}\left(x_{3}\right)+\phi_{P}^{T}\left(x_{3}\right)\right)\left(\eta \phi_{s}(z)+f_{+} f_{-} \phi_{t}(z)\right)\right] E_{n}\left(t_{d}^{V}\right) h_{d}^{V}\left(\alpha_{e}^{V}, \beta_{d}^{V}, b_{B}, b_{3}\right)\right\}, \\
& M_{e V}^{S P}=-32 \pi C_{F} m_{B}^{4} / \sqrt{6} / \sqrt{\eta} \int_{0}^{1} d x_{B} d z d x_{3} \int_{0}^{\infty} b_{B} d b_{B} b_{3} d b_{3} \phi_{B}\left(x_{B}, b_{B}\right) \phi_{P}^{A}\left(x_{3}\right) \\
& \times\left\{\left[\left(f_{-} g_{-}-f_{+} g_{+}\right)\left(g_{-}+g_{+}\left(1-x_{3}\right)-x_{B}+f_{+} z\right) \sqrt{\eta} \phi_{0}(z)+\left(-g_{-}\left(g_{+}\left(-2+x_{3}\right)+x_{B}\right)\right.\right.\right. \\
& \left.\left.+f_{+} g_{+} z\right) \eta \phi_{s}(z)+f_{-} f_{+}\left(g_{-}\left(g_{+} x_{3}+x_{B}\right)+f_{+} g_{+} z\right) \phi_{t}(z)\right] \\
& \times E_{n}\left(t_{c}^{V}\right) h_{c}^{V}\left(\alpha_{e}^{V}, \beta_{c}^{V}, b_{B}, b_{3}\right) \\
& -\left[-f_{+}\left(g_{-}+g_{+}\right)\left(g_{+} x_{3}-x_{B}-f_{-} z\right) \sqrt{\eta} \phi_{0}(z)+\left(g_{-} g_{+} x_{3}-g_{-} x_{B}+f_{+} g_{+} z\right)\right. \\
& \left.\left.\times \eta \phi_{s}(z)-f_{-} f_{+}\left(g_{-}\left(x_{B}-g_{+} x_{3}\right)+f_{+} g_{+} z\right) \phi_{t}(z)\right] E_{n}\left(t_{d}^{V}\right) h_{d}^{V}\left(\alpha_{e}^{V}, \beta_{d}^{V}, b_{B}, b_{3}\right)\right\} \text {, }
\end{aligned}
$$




$$
\begin{aligned}
& F_{a V}^{L L}=-8 \pi C_{F} m_{B}^{4} f_{B} / \sqrt{6} \int_{0}^{1} d z d x_{3} \int_{0}^{\infty} b d b b_{3} d b_{3} \\
& \times\left\{\left[\left(\left(f_{-}^{2} g_{-}+f_{-} g_{-} g_{+}+f_{+} g_{+}\left(f_{+}(z-1)-g_{-}\right)\right) \sqrt{\eta} \phi_{0}(z) \phi_{P}^{A}\left(x_{3}\right)+2 r_{03}\left(f_{-}+f_{+}+g_{-}+g_{+}-f_{+} z\right) \eta \phi_{s}(z) \phi_{P}^{P}\left(x_{3}\right)\right.\right.\right. \\
& \left.\left.+2 f_{-} f_{+} r_{03}\left(f_{-}-g_{-}+g_{+}+f_{+}(z-1)\right) \phi_{t}(z) \phi_{P}^{P}\left(x_{3}\right)\right) / \sqrt{\eta}\right] E_{a}\left(t_{e}^{V}\right) h_{e}^{V}\left(\alpha_{a}^{V}, \beta_{e}^{V}, b, b_{3}\right) S_{t}(z) \\
& -\left[f_{+}\left(f_{-}\left(g_{-}-g_{+}\right)-g_{+}^{2} x_{3}\right) \phi_{0}(z) \phi_{P}^{A}\left(x_{3}\right)+2 r_{03} \sqrt{\eta}\left(f_{+}\left(\phi_{P}^{P}\left(x_{3}\right)-\phi_{P}^{T}\left(x_{3}\right)\right)\right.\right. \\
& \left.\left.\left.+\left(f_{-}+g_{+} x_{3}\right)\left(\phi_{P}^{P}\left(x_{3}\right)+\phi_{P}^{T}\left(x_{3}\right)\right)\right) \phi_{s}(z)\right] E_{a}\left(t_{f}^{V}\right) h_{f}^{V}\left(\alpha_{a}^{V}, \beta_{f}^{V}, b, b_{3}\right) S_{t}\left(\left|f_{-}+x_{3} g_{+}\right|\right)\right\} \\
& F_{a V}^{L R}=-F_{a V}^{L L} \\
& F_{a V}^{S P}=16 \pi C_{F} m_{B}^{4} f_{B} / \sqrt{6} \int_{0}^{1} d z d x_{3} \int_{0}^{\infty} b d b b_{3} d b_{3} \\
& \times\left\{\left[\left(2 r_{03}\left(f_{+}\left(g_{+}+f_{-} z\right)-f_{-} g_{-}\right) \sqrt{\eta} \phi_{0}(z) \phi_{P}^{P}\left(x_{3}\right)-\left(f_{-} g_{-}+g_{+}\left(f_{+}(1-z)+2 g_{-}\right)\right) \eta \phi_{s}(z) \phi_{P}^{A}\left(x_{3}\right)\right.\right.\right. \\
& \left.\left.+f_{-} f_{+}\left(f_{-} g_{-}+f_{+} g_{+}(z-1)\right) \phi_{t}(z) \phi_{P}^{A}\left(x_{3}\right)\right) / \sqrt{\eta}\right] E_{a}\left(t_{e}^{V}\right) h_{e}^{V}\left(\alpha_{a}^{V}, \beta_{e}^{V}, b, b_{3}\right) S_{t}(z) \\
& +\left[f_{+} r_{03} \phi_{0}(z)\left(g_{+} x_{3}\left(\phi_{P}^{P}\left(x_{3}\right)-\phi_{P}^{T}\left(x_{3}\right)\right)-2 f_{-} \phi_{P}^{T}\left(x_{3}\right)\right)-2\left(f_{-} g_{-}+g_{+}\left(f_{+}+g_{-} x_{3}\right)\right) \sqrt{\eta} \phi_{s}(z) \phi_{P}^{A}\left(x_{3}\right)\right] \\
& \left.\times E_{a}\left(t_{f}^{V}\right) h_{f}^{V}\left(\alpha_{a}^{V}, \beta_{f}^{V}, b, b_{3}\right) S_{t}\left(\left|f_{-}+x_{3} g_{+}\right|\right)\right\}, \\
& M_{a V}^{L L}=32 \pi C_{F} m_{B}^{4} / \sqrt{6} / \sqrt{\eta} \int_{0}^{1} d x_{B} d z d x_{3} \int_{0}^{\infty} b_{B} d b_{B} b d b \phi_{B}\left(x_{B}, b_{B}\right) \\
& \times\left\{\left[\left(f_{+} g_{+}+f_{-} g_{-}\left(g_{-}+g_{+}-1\right)+f_{+}\left(g_{-}+g_{+}\right)\left(g_{+}\left(x_{3}-1\right)+x_{B}\right)\right.\right.\right. \\
& \left.+f_{-} f_{+}\left(g_{-}+g_{+}\right) z\right) \sqrt{\eta} \phi_{0}(z) \phi_{P}^{A}\left(x_{3}\right)+r_{03} \eta\left(\left(g_{-}+g_{+}\left(1-x_{3}\right)-x_{B}+f_{+} z-4\right) \phi_{P}^{P}\left(x_{3}\right)\right. \\
& \left.-\left(g_{-}+g_{+}\left(x_{3}-1\right)+x_{B}+f_{+} z\right) \phi_{P}^{T}\left(x_{3}\right)\right) \phi_{s}(z)+f_{-} f_{+} r_{03}\left(\left(f_{+} z+g_{-}\right)\left(\phi_{P}^{P}\left(x_{3}\right)-\phi_{P}^{T}\left(x_{3}\right)\right)\right. \\
& \left.\left.+\left(x_{B}+g_{+}\left(x_{3}-1\right)\right)\left(\phi_{P}^{P}\left(x_{3}\right)+\phi_{P}^{T}\left(x_{3}\right)\right)\right) \phi_{t}(z)\right] E_{n}\left(t_{g}^{V}\right) h_{g}^{V}\left(\alpha_{a}^{V}, \beta_{g}^{V}, b_{B}, b\right) \\
& +\left[\left(f_{-}-f_{+}\right)\left(f_{+} g_{+}(1-z)+g_{-}\left(f_{-}-x_{B}+g_{+} x_{3}\right)\right) \sqrt{\eta} \phi_{0}(z) \phi_{P}^{A}\left(x_{3}\right)\right. \\
& +\left(r_{03} \eta \phi_{s}(z)+f_{-} f_{+} r_{03} \phi_{t}(z)\right)\left(f_{-}+g_{+} x_{3}-x_{B}\right)\left(\phi_{P}^{P}\left(x_{3}\right)-\phi_{P}^{T}\left(x_{3}\right)\right) \\
& \left.\left.+\left(f_{-} f_{+} r_{03} \phi_{t}(z)-r_{03} \eta \phi_{s}(z)\right) f_{+}(z-1)\left(\phi_{P}^{P}\left(x_{3}\right)+\phi_{P}^{T}\left(x_{3}\right)\right)\right] E_{n}\left(t_{h}^{V}\right) h_{h}^{V}\left(\alpha_{a}^{V}, \beta_{h}^{V}, b_{B}, b\right)\right\}, \\
& M_{a V}^{L R}=-32 \pi C_{F} m_{B}^{4} / \sqrt{6} / \sqrt{\eta} \int_{0}^{1} d x_{B} d z d x_{3} \int_{0}^{\infty} b_{B} d b_{B} b d b \phi_{B}\left(x_{B}, b_{B}\right) \\
& \times\left\{\left[r_{03} \sqrt{\eta} \phi_{0}(z)\left(f_{-}\left(1+g_{-}+f_{+} z\right)\left(\phi_{P}^{P}\left(x_{3}\right)-\phi_{P}^{T}\left(x_{3}\right)\right)+f_{+}\left(g_{+}\left(x_{3}-1\right)-1+x_{B}\right)\left(\phi_{P}^{P}\left(x_{3}\right)+\phi_{P}^{T}\left(x_{3}\right)\right)\right)\right.\right. \\
& +\left(g_{-}\left(g_{+}\left(x_{3}-2\right)-1+x_{B}\right)-g_{+}\left(1+f_{+} z\right)\right) \eta \phi_{P}^{A}\left(x_{3}\right) \phi_{s}(z) \\
& \left.+f_{-} f_{+}\left(g_{+}+g_{-}\left(g_{+} x_{3}+x_{B}-1\right)+f_{+} g_{+} z\right) \phi_{P}^{A}\left(x_{3}\right) \phi_{t}(z)\right] E_{n}\left(t_{g}^{V}\right) h_{g}^{V}\left(\alpha_{a}^{V}, \beta_{g}^{V}, b_{B}, b\right) \\
& -\left[f_{+} r_{03} \sqrt{\eta} \phi_{0}(z)\left(\left(g_{+} x_{3}-x_{B}\right)\left(\phi_{P}^{P}\left(x_{3}\right)+\phi_{P}^{T}\left(x_{3}\right)\right)+f_{-}\left(z \phi_{P}^{P}\left(x_{3}\right)+(2-z) \phi_{P}^{T}\left(x_{3}\right)\right)\right)\right. \\
& +\left(f_{+} g_{+}(1-z)+g_{-}\left(f_{-}+g_{+} x_{3}-x_{B}\right)\right) \eta \phi_{P}^{A}\left(x_{3}\right) \phi_{s}(z)+f_{-} f_{+}\left(g_{-}\left(f_{-}+g_{+} x_{3}-x_{B}\right)\right. \\
& \left.\left.\left.+f_{+} g_{+}(-1+z)\right) \phi_{P}^{A}\left(x_{3}\right) \phi_{t}(z)\right] E_{n}\left(t_{h}^{V}\right) h_{h}^{V}\left(\alpha_{a}^{V}, \beta_{h}^{V}, b_{B}, b\right)\right\}
\end{aligned}
$$




$$
\begin{aligned}
M_{a V}^{S P}= & 32 \pi C_{F} m_{B}^{4} / \sqrt{6} / \sqrt{\eta} \int_{0}^{1} d x_{B} d z d x_{3} \int_{0}^{\infty} b_{B} d b_{B} b d b \phi_{B}\left(x_{B}, b_{B}\right) \\
& \times\left\{\left[\left(g_{-}\left(f_{-}\left(1+g_{+}\left(x_{3}-2\right)+x_{B}\right)-f_{+}\left(g_{+}\left(x_{3}-2\right)+x_{B}\right)\right)\right.\right.\right. \\
& \left.+f_{+} g_{+}\left(\left(f_{+}-f_{-}\right) z-1\right)\right) \sqrt{\eta} \phi_{0}(z) \phi_{P}^{A}\left(x_{3}\right)-r_{03} \eta\left(\left(g_{-}+g_{+}\left(1-x_{3}\right)-x_{B}+f_{+} z-4\right) \phi_{P}^{P}\left(x_{3}\right)\right. \\
& \left.+\left(g_{-}+g_{+}\left(x_{3}-1\right)+x_{B}+f_{+} z\right) \phi_{P}^{T}\left(x_{3}\right)\right) \phi_{s}(z)+f_{-} f_{+} r_{03}\left(\left(g_{-}+g_{+}\left(x_{3}-1\right)+x_{B}\right.\right. \\
& \left.\left.\left.+f_{+} z\right) \phi_{P}^{P}\left(x_{3}\right)+\left(g_{-}+g_{+}\left(1-x_{3}\right)-x_{B}+f_{+} z\right) \phi_{P}^{T}\left(x_{3}\right)\right) \phi_{t}(z)\right] E_{n}\left(t_{g}^{V}\right) h_{g}^{V}\left(\alpha_{a}^{V}, \beta_{g}^{V}, b_{B}, b\right) \\
& +\left[f_{+}\left(g_{-}+g_{+}\right)\left(g_{+} x_{3}-x_{B}+f_{-} z\right) \sqrt{\eta} \phi_{0}(z) \phi_{P}^{A}\left(x_{3}\right)+\left(r_{03} \eta \phi_{s}(z)+f_{-} f_{+} r_{03} \phi_{t}(z)\right) f_{+}(z-1)\right. \\
& \left.\times\left(\phi_{P}^{P}\left(x_{3}\right)-\phi_{P}^{T}\left(x_{3}\right)\right)+\left(f_{-} f_{+} r_{03} \phi_{t}(z)-r_{03} \eta \phi_{s}(z)\right)\left(g_{+} x_{3}-x_{B}+f_{-}\right)\left(\phi_{P}^{P}\left(x_{3}\right)+\phi_{P}^{T}\left(x_{3}\right)\right)\right] \\
& \left.\times E_{n}\left(t_{h}^{V}\right) h_{h}^{V}\left(\alpha_{a}^{V}, \beta_{h}^{V}, b_{B}, b\right)\right\},
\end{aligned}
$$

$$
\begin{aligned}
F_{e P}^{L L}= & 8 \pi C_{F} m_{B}^{4} F\left(w^{2}\right) \int_{0}^{1} d x_{B} d x_{3} \int_{0}^{\infty} b_{B} d b_{B} b_{3} d b_{3} \phi_{B}\left(x_{B}, b_{B}\right) \\
& \times\left\{\left[\left(f_{-} g_{-}-f_{+} g_{+}\left(1+g_{+} x_{3}\right)\right) \phi_{P}^{A}\left(x_{3}\right)+r_{03}\left(f_{-}+f_{+}\left(2 g_{+} x_{3}-1\right)\right) \phi_{P}^{P}\left(x_{3}\right)\right.\right. \\
& \left.-r_{03}\left(f_{-}+f_{+}-2 f_{+} g_{+} x_{3}\right) \phi_{P}^{T}\left(x_{3}\right)\right] E_{e}\left(t_{a}^{P}\right) h_{a}^{P}\left(\alpha_{e}^{P}, \beta_{a}^{P}, b_{B}, b_{3}\right) S_{t}\left(x_{3}\right) \\
& -\left[-g_{+}\left(f_{+} g_{-}+f_{-}\left(x_{B}-g_{-}\right)\right) \phi_{P}^{A}\left(x_{3}\right)+2 r_{03}\left(f_{+} g_{+}+f_{-}\left(x_{B}-g_{-}\right)\right) \phi_{P}^{P}\left(x_{3}\right)\right] \\
& \left.\times E_{e}\left(t_{b}^{P}\right) h_{b}^{P}\left(\alpha_{e}^{P}, \beta_{b}^{P}, b_{B}, b_{3}\right) S_{t}\left(\left|x_{B}-g_{-}\right|\right)\right\}
\end{aligned}
$$

$$
F_{e P}^{L R}=F_{e P}^{L L},
$$

$$
F_{e P}^{S P}=0
$$

$$
\begin{aligned}
M_{e P}^{L L}= & 32 \pi C_{F} m_{B}^{4} / \sqrt{6} \int_{0}^{1} d x_{B} d z d x_{3} \int_{0}^{\infty} b_{B} d b_{B} b d b \phi_{B}\left(x_{B}, b_{B}\right) \phi_{0}(z) \\
& \times\left\{\left[\left(f_{-}-f_{+}\right)\left(f_{-} g_{-}+g_{+}\left(f_{+}(1-z)+g_{-} x_{3}-x_{B}\right)\right) \phi_{P}^{A}\left(x_{3}\right)+r_{03}\left(f_{+} g_{+} x_{3}+f_{-} x_{B}\right.\right.\right. \\
& \left.\left.+f_{-} f_{+} z\right) \phi_{P}^{P}+r_{03}\left(-f_{+} g_{+} x_{3}+f_{-}\left(x_{B}+f_{+}(z-2)\right)\right) \phi_{P}^{T}\left(x_{3}\right)\right] E_{n}\left(t_{c}^{P}\right) h_{c}^{P}\left(\alpha_{e}^{P}, \beta_{c}^{P}, b_{B}, b\right) \\
& -\left[\left(f_{-} g_{-}-f_{+} g_{+}\right)\left(f_{+} z+g_{+} x_{3}-x_{B}\right) \phi_{P}^{A}\left(x_{3}\right)+r_{03}\left(f_{+} g_{+} x_{3}+f_{-}\left(x_{B}-f_{+} z\right) \phi_{P}^{P}\left(x_{3}\right)\right.\right. \\
& \left.\left.\left.+r_{03}\left(f_{+} g_{+} x_{3}-f_{-}\left(x_{B}-f_{+} z\right)\right)\right) \phi_{P}^{T}\left(x_{3}\right)\right] E_{n}\left(t_{d}^{P}\right) h_{d}^{P}\left(\alpha_{e}^{P}, \beta_{d}^{P}, b_{B}, b\right)\right\},
\end{aligned}
$$




$$
\begin{aligned}
& M_{e P}^{L R}=32 \pi C_{F} m_{B}^{4} / \sqrt{6} / \sqrt{\eta} \int_{0}^{1} d x_{B} d z d x_{3} \int_{0}^{\infty} b_{B} d b_{B} b d b \phi_{B}\left(x_{B}, b_{B}\right) \\
& \times\left\{\left[-\left(f_{-} g_{-}+g_{+}\left(f_{+}+g_{-} x_{3}-x_{B}-f_{+} z\right)\right) \eta \phi_{P}^{A}\left(x_{3}\right) \phi_{s}(z)+f_{-} f_{+}\left(f_{-} g_{-}+g_{+}\left(g_{-} x_{3}+x_{B}\right.\right.\right.\right. \\
& \left.\left.+f_{+}(-1+z)\right)\right) \phi_{P}^{A}\left(x_{3}\right) \phi_{t}(z)+r_{03} \phi_{P}^{P}\left(x_{3}\right)\left(-\left(f_{-}+f_{+}(1-z)+g_{+} x_{3}-x_{B}\right) \eta \phi_{s}(z)+f_{-} f_{+}\left(f_{-}+g_{+} x_{3}\right.\right. \\
& \left.\left.+x_{B}+f_{+}(-1+z)\right) \phi_{t}(z)\right)+r_{03} \phi_{P}^{T}\left(x_{3}\right)\left(-\left(f_{-}+g_{+} x_{3}+x_{B}+f_{+}(-1+z)\right) \eta \phi_{s}(z)\right. \\
& \left.\left.+f_{-} f_{+}\left(f_{-}+f_{+}(1-z)+g_{+} x_{3}-x_{B}\right) \phi_{t}(z)\right)\right] E_{n}\left(t_{c}^{P}\right) h_{c}^{P}\left(\alpha_{e}^{P}, \beta_{c}^{P}, b_{B}, b\right) \\
& +\left[g_{+} \phi_{P}^{A}\left(x_{3}\right)\left(\left(g_{-} x_{3}-x_{B}+f_{+} z\right) \eta \phi_{s}(z)+f_{-} f_{+}\left(g_{-} x_{3}+x_{B}-f_{+} z\right) \phi_{t}(z)\right)\right. \\
& +r_{03} \phi_{P}^{P}\left(x_{3}\right)\left(\left(g_{+} x_{3}-x_{B}+f_{+} z\right) \eta \phi_{s}(z)+f_{-} f_{+}\left(g_{+} x_{3}+x_{B}-f_{+} z\right) \phi_{t}(z)\right) \\
& \left.\left.+r_{03} \phi_{P}^{T}\left(x_{3}\right)\left(\left(g_{+} x_{3}+x_{B}-f_{+} z\right) \eta \phi_{s}(z)+f_{-} f_{+}\left(g_{+} x_{3}-x_{B}+f_{+} z\right) \phi_{t}(z)\right)\right] E_{n}\left(t_{d}^{P}\right) h_{d}^{P}\left(\alpha_{e}^{P}, \beta_{d}^{P}, b_{B}, b\right)\right\}, \\
& M_{e P}^{S P}=32 \pi C_{F} m_{B}^{4} / \sqrt{6} \int_{0}^{1} d x_{B} d z d x_{3} \int_{0}^{\infty} b_{B} d b_{B} b d b \phi_{B}\left(x_{B}, b_{B}\right) \phi_{0}(z) \\
& \times\left\{\left[\left(f_{-} g_{-}-f_{+} g_{+}\right)\left(f_{-}+f_{+}(1-z)+g_{+} x_{3}-x_{B}\right) \phi_{P}^{A}\left(x_{3}\right)+r_{03}\left(f_{+} g_{+} x_{3}+f_{-} x_{B}\right.\right.\right. \\
& \left.\left.+f_{-} f_{+} z\right) \phi_{P}^{P}\left(x_{3}\right)+r_{03}\left(f_{+} g_{+} x_{3}-f_{-}\left(x_{B}+f_{+}(z-2)\right)\right) \phi_{P}^{T}\left(x_{3}\right)\right] E_{n}\left(t_{c}^{P}\right) h_{c}^{P}\left(\alpha_{e}^{P}, \beta_{c}^{P}, b_{B}, b\right) \\
& -\left[\left(f_{-}-f_{+}\right) g_{+}\left(g_{-} x_{3}-x_{B}+f_{+} z\right) \phi_{P}^{A}\left(x_{3}\right)+r_{03}\left(f_{+} g_{+} x_{3}+f_{-} x_{B}-f_{-} f_{+} z\right) \phi_{P}^{P}\left(x_{3}\right)\right. \\
& \left.\left.-r_{03}\left(f_{+} g_{+} x_{3}+f_{-}\left(f_{+} z-x_{B}\right)\right) \phi_{P}^{T}\left(x_{3}\right)\right] E_{n}\left(t_{d}^{P}\right) h_{d}^{P}\left(\alpha_{e}^{P}, \beta_{d}^{P}, b_{B}, b\right)\right\} \text {, }
\end{aligned}
$$

$$
\begin{aligned}
F_{a P}^{L L}= & -8 \pi C_{F} m_{B}^{4} f_{B} \int_{0}^{1} d z d x_{3} \int_{0}^{\infty} b d b b_{3} d b_{3} \\
& \times\left\{\left[\left(f_{-} g_{-}+f_{+} g_{+}\left(g_{+} x_{3}-1\right)\right) \phi_{0}(z) \phi_{P}^{A}\left(x_{3}\right)+2 r_{03}\left(g_{+} x_{3}-2\right) \sqrt{\eta} \phi_{P}^{P}\left(x_{3}\right) \phi_{s}(z)-2 g_{+} r_{03} x_{3} \sqrt{\eta} \phi_{P}^{T}\left(x_{3}\right) \phi_{s}(z)\right]\right. \\
& \times E_{a}\left(t_{e}^{P}\right) h_{e}^{P}\left(\alpha_{a}^{P}, \beta_{e}^{P}, b, b_{3}\right) S_{t}\left(x_{3}\right) \\
& +\left[g_{+}\left(f_{+}\left(g_{-}+f_{+} z\right)-f_{-} g_{-}\right) \phi_{0}(z) \phi_{P}^{A}\left(x_{3}\right)+2 r_{03} \phi_{P}^{P}\left(x_{3}\right)\left(\left(g_{-}+g_{+}+f_{+} z\right) \sqrt{\eta} \phi_{s}(z)\right.\right. \\
& \left.\left.\left.+f_{-} f_{+}\left(g_{-}-g_{+}+f_{+} z\right) \phi_{t}(z) / \sqrt{\eta}\right)\right] E_{a}\left(t_{f}^{P}\right) h_{f}^{P}\left(\alpha_{a}^{P}, \beta_{f}^{P}, b, b_{3}\right) S_{t}\left(\left|g_{-}+z f_{+}\right|\right)\right\}
\end{aligned}
$$

$$
F_{a P}^{L R}=-F_{a P}^{L L}
$$

$$
\begin{aligned}
F_{a P}^{S P}= & 16 \pi C_{F} m_{B}^{4} f_{B} \int_{0}^{1} d z d x_{3} \int_{0}^{\infty} b d b b_{3} d b_{3} \\
& \times\left\{\left[2\left(g_{-}\left(g_{+} x_{3}-1\right)-g_{+}\right) \sqrt{\eta} \phi_{P}^{A}\left(x_{3}\right) \phi_{s}(z)+r_{03}\left(f_{-}+f_{+}\left(g_{+} x_{3}-1\right)\right) \phi_{0}(z) \phi_{P}^{P}\left(x_{3}\right)-r_{03}\left(f_{-}+f_{+}\right.\right.\right. \\
& \left.\left.-f_{+} g_{+} x_{3}\right) \phi_{0}(z) \phi_{P}^{T}\left(x_{3}\right)\right] E_{a}\left(t_{e}^{P}\right) h_{e}^{P}\left(\alpha_{a}^{P}, \beta_{e}^{P}, b, b_{3}\right) S_{t}\left(x_{3}\right) \\
& +\left[\phi_{P}^{A}\left(x_{3}\right)\left(-2 g_{-} g_{+} \eta \phi_{s}(z)+f_{+} g_{+} z\left(f_{-} f_{+} \phi_{t}(z)-\eta \phi_{s}(z)\right)\right) / \sqrt{\eta}+2 r_{03}\left(-f_{+} g_{+}+f_{-}\left(g_{-}+f_{+} z\right)\right) \phi_{0}(z) \phi_{P}^{P}\left(x_{3}\right)\right] \\
& \left.\times E_{a}\left(t_{f}^{P}\right) h_{f}^{P}\left(\alpha_{a}^{P}, \beta_{f}^{P}, b, b_{3}\right) S_{t}\left(\left|g_{-}+z f_{+}\right|\right)\right\}
\end{aligned}
$$




$$
\begin{aligned}
M_{a P}^{L L}= & 32 \pi C_{F} m_{B}^{4} / \sqrt{6} / \sqrt{\eta} \int_{0}^{1} d x_{B} d z d x_{3} \int_{0}^{\infty} b_{B} d b_{B} b d b \phi_{B}\left(x_{B}, b_{B}\right) \\
& \times\left\{\left[\left(f_{-}^{2} g_{-}-f_{-}\left(g_{-}\left(1+f_{+}-g_{+} x_{3}\right)+g_{+}\left(x_{B}+f_{+}(z-1)\right)\right)+f_{+} g_{+}\left(1-g_{-} x_{3}+x_{B}\right.\right.\right.\right. \\
& \left.\left.+f_{+}(z-1)\right)\right) \sqrt{\eta} \phi_{0}(z) \phi_{P}^{A}\left(x_{3}\right)+r_{03} \phi_{P}^{P}\left(x_{3}\right)\left(-\left(-4+f_{-}+f_{+}(1-z)+g_{+} x_{3}-x_{B}\right) \eta \phi_{s}(z)\right. \\
& \left.+f_{-} f_{+}\left(f_{-}+g_{+} x_{3}+x_{B}+f_{+}(z-1)\right) \phi_{t}(z)\right)+r_{03} \phi_{P}^{T}\left(x_{3}\right)\left(-\left(f_{-}+g_{+} x_{3}+x_{B}+f_{+}(z-1)\right) \eta \phi_{s}(z)\right. \\
& \left.\left.+f_{-} f_{+}\left(f_{-}+f_{+}(1-z)+g_{+} x_{3}-x_{B}\right) \phi_{t}(z)\right)\right] E_{n}\left(t_{g}^{P}\right) h_{g}^{P}\left(\alpha_{a}^{P}, \beta_{g}^{P}, b_{B}, b\right) \\
& +\left[\left(g_{-}+g_{+}\right)\left(f_{+} g_{+}\left(x_{3}-1\right)+f_{-}\left(g_{-}-x_{B}+f_{+} z\right)\right) \sqrt{\eta} \phi_{0}(z) \phi_{P}^{A}\left(x_{3}\right)+r_{03} \phi_{P}^{P}\left(x_{3}\right)\left(-\left(g_{-}+g_{+}\left(1-x_{3}\right)\right.\right.\right. \\
& \left.\left.-x_{B}+f_{+} z\right) \eta \phi_{s}(z)+f_{-} f_{+}\left(g_{-}+g_{+}\left(x_{3}-1\right)-x_{B}+f_{+} z\right) \phi_{t}(z)\right)+r_{03} \phi_{P}^{T}\left(x_{3}\right)\left(\left(-g_{-}+g_{+}\left(1-x_{3}\right)\right.\right. \\
& \left.\left.\left.+x_{B}-f_{+} z\right) \eta \phi_{s}(z)+f_{-} f_{+}\left(g_{-}+g_{+}\left(1-x_{3}\right)-x_{B}+f_{+} z\right) \phi_{t}(z)\right)\right] \\
& \left.\times E_{n}\left(t_{h}^{P}\right) h_{h}^{P}\left(\alpha_{a}^{P}, \beta_{h}^{P}, b_{B}, b\right)\right\},
\end{aligned}
$$

$$
\begin{aligned}
M_{a P}^{L R}= & 32 \pi C_{F} m_{B}^{4} / \sqrt{6} / \sqrt{\eta} \int_{0}^{1} d x_{B} d z d x_{3} \int_{0}^{\infty} b_{B} d b_{B} b d b \phi_{B}\left(x_{B}, b_{B}\right) \\
& \times\left\{\left[\phi_{P}^{A}\left(x_{3}\right)\left(g_{-}\left(1+f_{-}+g_{+} x_{3}\right)\left(f_{-} f_{+} \phi_{t}(z)-\eta \phi_{s}(z)\right)+g_{+}\left(-1+x_{B}+f_{+}(z-1)\right)\left(\eta \phi_{s}(z)+f_{-} f_{+} \phi_{t}(z)\right)\right)\right.\right. \\
& +r_{03}\left(f_{+}\left(1+g_{+} x_{3}\right)+f_{-}\left(x_{B}+f_{+} z-1\right)\right) \sqrt{\eta} \phi_{0}(z) \phi_{P}^{P}\left(x_{3}\right)+r_{03}\left(-f_{+}\left(1+g_{+} x_{3}\right)\right. \\
& \left.\left.+f_{-}\left(-1+x_{B}+f_{+}(z-2)\right)\right) \sqrt{\eta} \phi_{0}(z) \phi_{P}^{T}\left(x_{3}\right)\right] E_{n}\left(t_{g}^{P}\right) h_{g}^{P}\left(\alpha_{a}^{P}, \beta_{g}^{P}, b_{B}, b\right) \\
& -\left[g_{+} \phi_{P}^{A}\left(x_{3}\right)\left(-\left(g_{-}\left(x_{3}-2\right)+x_{B}-f_{+} z\right) \eta \phi_{s}(z)+f_{-} f_{+}\left(g_{-} x_{3}-x_{B}+f_{+} z\right) \phi_{t}(z)\right)\right. \\
& +r_{03}\left(f_{+} g_{+}\left(x_{3}-1\right)+f_{-}\left(g_{-}-x_{B}+f_{+} z\right)\right) \sqrt{\eta} \phi_{0}(z) \phi_{P}^{P}\left(x_{3}\right)+r_{03}\left(-f_{+} g_{+}\left(x_{3}-1\right)\right. \\
& \left.\left.\left.+f_{-}\left(g_{-}-x_{B}+f_{+} z\right)\right) \sqrt{\eta} \phi_{0}(z) \phi_{P}^{T}\left(x_{3}\right)\right] E_{n}\left(t_{h}^{P}\right) h_{h}^{P}\left(\alpha_{a}^{P}, \beta_{h}^{P}, b_{B}, b\right)\right\}
\end{aligned}
$$

$$
\begin{aligned}
M_{a P}^{S P}= & 32 \pi C_{F} m_{B}^{4} / \sqrt{6} / \sqrt{\eta} \int_{0}^{1} d x_{B} d z d x_{3} \int_{0}^{\infty} b_{B} d b_{B} b d b \phi_{B}\left(x_{B}, b_{B}\right) \\
& \times\left\{\left[\left(f_{+} g_{+}\left(-1+\left(g_{-}+g_{+}\right) x_{3}\right)+f_{-}\left(g_{-}\left(1+x_{B}\right)+g_{+} x_{B}+f_{+}\left(g_{-}+g_{+}\right) z\right)\right) \sqrt{\eta} \phi_{0}(z) \phi_{P}^{A}\left(x_{3}\right)\right.\right. \\
& +r_{03} \phi_{P}^{P}\left(x_{3}\right)\left(\left(-4+f_{-}+f_{+}(1-z)+g_{+} x_{3}-x_{B}\right) \eta \phi_{s}(z)+f_{-} f_{+}\left(f_{-}+g_{+} x_{3}+x_{B}+f_{+}(-1\right.\right. \\
& \left.+z)) \phi_{t}(z)\right)+r_{03} \phi_{P}^{T}\left(x_{3}\right)\left(-\left(f_{-}+g_{+} x_{3}+x_{B}+f_{+}(z-1)\right) \eta \phi_{s}(z)-f_{-} f_{+}\left(f_{-}+f_{+}(1-z)\right.\right. \\
& \left.\left.\left.+g_{+} x_{3}-x_{B}\right) \phi_{t}(z)\right)\right] E_{n}\left(t_{g}^{P}\right) h_{g}^{P}\left(\alpha_{a}^{P}, \beta_{g}^{P}, b_{B}, b\right) \\
& +\left[\left(f_{-}-f_{+}\right) g_{+}\left(g_{-}\left(x_{3}-2\right)+x_{B}-f_{+} z\right) \sqrt{\eta} \phi_{0}(z) \phi_{P}^{A}\left(x_{3}\right)+r_{03} \phi_{P}^{P}\left(x_{3}\right)\left(\left(g_{-}+g_{+}\left(1-x_{3}\right)-x_{B}\right.\right.\right. \\
& \left.\left.+f_{+} z\right) \eta \phi_{s}(z)+f_{-} f_{+}\left(g_{-}+g_{+}\left(x_{3}-1\right)-x_{B}+f_{+} z\right) \phi_{t}(z)\right)+r_{03} \phi_{P}^{T}\left(x_{3}\right)\left(\left(-g_{-}+g_{+}\left(1-x_{3}\right)\right.\right. \\
& \left.\left.\left.\left.+x_{B}-f_{+} z\right) \eta \phi_{s}(z)-f_{-} f_{+}\left(g_{-}+g_{+}\left(1-x_{3}\right)-x_{B}+f_{+} z\right) \phi_{t}(z)\right)\right] E_{n}\left(t_{h}^{P}\right) h_{h}^{P}\left(\alpha_{a}^{P}, \beta_{h}^{P}, b_{B}, b\right)\right\},
\end{aligned}
$$

with the color factor $C_{F}=4 / 3$ and the mass ratio $r_{03}=m_{03} / m_{B_{(s)}}$.

The evolution factors $E_{i}(t), i=e, a, n$ in the above factorization formulas are written as

$$
\begin{aligned}
& E_{e}(t)=\alpha_{s}(t) \exp \left[-S_{B}(t)-S_{V}(t)\right] \\
& E_{a}(t)=\alpha_{s}(t) \exp \left[-S_{P}(t)-S_{V}(t)\right] \\
& E_{n}(t)=\alpha_{s}(t) \exp \left[-S_{B}(t)-S_{V}(t)-S_{P}(t)\right]
\end{aligned}
$$

where the Sudakov exponents $S_{B, P, V}$ are given by 


$$
\begin{aligned}
& S_{B}=s\left(x_{B} \frac{m_{B}}{\sqrt{2}}, b_{B}\right)+\frac{5}{3} \int_{1 / b_{B}}^{t} \frac{d \bar{\mu}}{\bar{\mu}} \gamma_{q}\left(\alpha_{s}(\bar{\mu})\right), \\
& S_{V}=s\left(\frac{m_{B}}{\sqrt{2}} z f_{+}, b\right)+s\left(\frac{m_{B}}{\sqrt{2}}(1-z) f_{+}, b\right)+2 \int_{1 / b}^{t} \frac{d \bar{\mu}}{\bar{\mu}} \gamma_{q}\left(\alpha_{s}(\bar{\mu})\right), \\
& S_{P}=s\left(\frac{m_{B}}{\sqrt{2}} x_{3} g_{+}, b_{3}\right)+s\left(\frac{m_{B}}{\sqrt{2}}\left(1-x_{3}\right) g_{+}, b_{3}\right)+2 \int_{1 / b_{3}}^{t} \frac{d \bar{\mu}}{\bar{\mu}} \gamma_{q}\left(\alpha_{s}(\bar{\mu})\right),
\end{aligned}
$$

with the quark anomalous dimension $\gamma_{q}=-\alpha_{s} / \pi$. The explicit expressions of the functions $s(Q, b)$ can be found in the Appendix of Ref. [49].

The threshold resummation factor $S_{t}(x)$ takes the form

$$
S_{t}(x)=\frac{2^{1+2 c} \Gamma(3 / 2+c)}{\sqrt{\pi} \Gamma(1+c)}[x(1-x)]^{c},
$$

where $c=0.3$ is adopted in our numerical analysis.

The hard functions $h_{i}^{V(P)}, i=a-h$, in the factorization formulas are written as

$$
\begin{aligned}
& h_{i}^{V(P)}\left(\alpha_{j}^{V(P)}, \beta_{i}^{V(P)}, b_{1}, b_{2}\right)=h_{1}\left(\alpha_{j}^{V(P)}, b_{1}\right) h_{2}\left(\beta_{i}^{V(P)}, b_{1}, b_{2}\right), \quad j=e, a, \\
& h_{1}\left(\alpha_{j}^{V(P)}, b_{1}\right)= \begin{cases}K_{0}\left(\sqrt{\alpha_{j}^{V(P)}} b_{1}\right), & \alpha_{j}^{V(P)}>0 \\
K_{0}\left(i \sqrt{-\alpha_{j}^{V(P)}} b_{1}\right), & \alpha_{j}^{V(P)}<0\end{cases} \\
& h_{2}\left(\beta_{i}^{V(P)}, b_{1}, b_{2}\right)= \begin{cases}\theta\left(b_{1}-b_{2}\right) I_{0}\left(\sqrt{\beta_{i}^{V(P)}} b_{2}\right) K_{0}\left(\sqrt{\beta_{i}^{V(P)}} b_{1}\right)+\left(b_{1} \leftrightarrow b_{2}\right), & \beta_{i}^{V(P)}>0, \\
\theta\left(b_{1}-b_{2}\right) I_{0}\left(\sqrt{-\beta_{i}^{V(P)}} b_{2}\right) K_{0}\left(i \sqrt{-\beta_{i}^{V(P)}} b_{1}\right)+\left(b_{1} \leftrightarrow b_{2}\right), & \beta_{i}^{V(P)}<0,\end{cases}
\end{aligned}
$$

with the Bessel function $K_{0}(i x)=\pi\left[-N_{0}(x)+i J_{0}(x)\right] / 2$, and the virtuality $\alpha_{j}^{V(P)}\left(\beta_{i}^{V(P)}\right)$ of the internal gluon (quark) in the diagrams:

$$
\begin{aligned}
& \alpha_{e}^{V}=f_{+} z x_{B}, \quad \alpha_{a}^{V}=f_{+}(z-1)\left(f_{-}+g_{+} x_{3}\right), \\
& \beta_{a}^{V}=z f_{+}, \quad \beta_{b}^{V}=\left(x_{B}-f_{-}\right) f_{+} \\
& \beta_{c}^{V}=\left(g_{-}+f_{+} z\right)\left(x_{B}+g_{+}\left(x_{3}-1\right)\right), \quad \beta_{d}^{V}=f_{+} z\left(x_{B}-g_{+} x_{3}\right), \\
& \beta_{e}^{V}=-\left(f_{-}+g_{+}\right)\left(f_{+}(1-z)+g_{-}\right), \quad \beta_{f}^{V}=-\left(f_{-}+g_{+} x_{3}\right) f_{+}, \\
& \beta_{g}^{V}=1+\left(g_{-}+f_{+} z\right)\left(x_{B}+g_{+}\left(x_{3}-1\right)\right), \quad \beta_{h}^{V}=f_{+}(z-1)\left(f_{-}-x_{B}+g_{+} x_{3}\right),
\end{aligned}
$$

and

$$
\begin{aligned}
& \alpha_{e}^{P}=g_{+} x_{3} x_{B}, \quad \alpha_{a}^{P}=g_{+}\left(x_{3}-1\right)\left(g_{-}+f_{+} z\right), \\
& \beta_{a}^{P}=x_{3} g_{+}, \quad \beta_{b}^{P}=\left(x_{B}-g_{-}\right) g_{+} \\
& \beta_{c}^{P}=\left(f_{-}+g_{+} x_{3}\right)\left(x_{B}+f_{+}(z-1)\right), \quad \beta_{d}^{P}=g_{+} x_{3}\left(x_{B}-f_{+} z\right), \\
& \beta_{e}^{P}=x_{3} g_{+}-1, \quad \beta_{f}^{P}=-\left(g_{-}+f_{+} z\right) g_{+}, \\
& \beta_{g}^{P}=1+\left(f_{-}+g_{+} x_{3}\right)\left(x_{B}+f_{+}(z-1)\right), \quad \beta_{h}^{P}=g_{+}\left(x_{3}-1\right)\left(g_{-}-x_{B}+f_{+} z\right) .
\end{aligned}
$$

The hard scales $t_{i}^{V(P)}, i=a-h$, are chosen as the maxima of the virtualities involved in the decays described by Fig. 1 (Fig. 2): 


$$
\begin{aligned}
t_{a, b}^{V} & =\max \left\{m_{B} \sqrt{\left|\alpha_{e}^{V}\right|}, m_{B} \sqrt{\left|\beta_{a, b}^{V}\right|}, 1 / b, 1 / b_{B}\right\}, \\
t_{c, d}^{V} & =\max \left\{m_{B} \sqrt{\left|\alpha_{e}^{V}\right|}, m_{B} \sqrt{\left|\beta_{c, d}^{V}\right|}, 1 / b_{3}, 1 / b_{B}\right\}, \\
t_{e, f}^{V} & =\max \left\{m_{B} \sqrt{\left|\alpha_{a}^{V}\right|}, m_{B} \sqrt{\left|\beta_{e, f}^{V}\right|}, 1 / b, 1 / b_{3}\right\}, \\
t_{g, h}^{V} & =\max \left\{m_{B} \sqrt{\left|\alpha_{a}^{V}\right|}, m_{B} \sqrt{\left|\beta_{g, h}^{V}\right|}, 1 / b, 1 / b_{B}\right\},
\end{aligned}
$$

and

$$
\begin{aligned}
& t_{a, b}^{P}=\max \left\{m_{B} \sqrt{\left|\alpha_{e}^{P}\right|}, m_{B} \sqrt{\left|\beta_{a, b}^{P}\right|}, 1 / b_{3}, 1 / b_{B}\right\}, \\
& t_{c, d}^{P}=\max \left\{m_{B} \sqrt{\left|\alpha_{e}^{P}\right|}, m_{B} \sqrt{\left|\beta_{c, d}^{P}\right|}, 1 / b, 1 / b_{B}\right\}, \\
& t_{e, f}^{P}=\max \left\{m_{B} \sqrt{\left|\alpha_{a}^{P}\right|}, m_{B} \sqrt{\left|\beta_{e, f}^{P}\right|}, 1 / b, 1 / b_{3}\right\}, \\
& t_{g, h}^{P}=\max \left\{m_{B} \sqrt{\left|\alpha_{a}^{P}\right|}, m_{B} \sqrt{\left|\beta_{g, h}^{P}\right|}, 1 / b, 1 / b_{B}\right\} .
\end{aligned}
$$

[1] C. H. Chen and H. n. Li, Three body nonleptonic $B$ decays in perturbative QCD, Phys. Lett. B 561, 258 (2003).

[2] Z. Rui, Y. Li, and W. F. Wang, The $S$-wave resonance contributions in the $B_{s}^{0}$ decays into $\psi(2 S, 3 S)$ plus pion pair, Eur. Phys. J. C 77, 199 (2017).

[3] Z. Rui, Y. Q. Li, and J. Zhang, Isovector scalar $a_{0}(980)$ and $a_{0}(1450)$ resonances in the $B \rightarrow \psi(K \bar{K}, \pi \eta)$ decays, Phys. Rev. D 99, 093007 (2019).

[4] Z. Rui, Y. Li, and H. Li, Studies of the resonance components in the $B_{s}$ decays into charmonia plus kaon pair, Eur. Phys. J. C 79, 792 (2019).

[5] Y. Xing and Z. P. Xing, $S$-wave contributions in $\bar{B}_{s}^{0} \rightarrow$ $\left(D^{0}, \bar{D}^{0}\right) \pi^{+} \pi^{-}$within perturbative QCD approach, Chin. Phys. C 43, 073103 (2019).

[6] Z. T. Zou, Y. Li, Q. X. Li, and X. Liu, Resonant contributions to three-body $B \rightarrow K K K$ decays in perturbative QCD approach, Eur. Phys. J. C 80, 394 (2020).

[7] Z. T. Zou, Y. Li, and X. Liu, Branching fractions and $C P$ asymmetries of the quasi-two-body decays in $B_{s} \rightarrow$ $K^{0}\left(\bar{K}^{0}\right) K^{ \pm} \pi^{\mp}$ within PQCD approach, Eur. Phys. J. C 80, 517 (2020).

[8] Z. T. Zou, L. Yang, Y. Li, and X. Liu, Study of quasi-twobody $B_{(s)} \rightarrow \phi\left(f_{0}(980) / f_{2}(1270) \rightarrow\right) \pi \pi$ decays in perturbative QCD approach, Eur. Phys. J. C 81, 91 (2021).

[9] Z. T. Zou, Y. Li, and H. n. Li, Is $f_{X}(1500)$ observed in the $B \rightarrow \pi(K) K K$ decays $\rho^{0}(1450)$ ?, Phys. Rev. D 103, 013005 (2021).

[10] Y. Li, A. J. Ma, W. F. Wang, and Z. J. Xiao, Quasi-two-body decays $B_{(s)} \rightarrow P \rho \rightarrow P \pi \pi$ in perturbative QCD approach, Phys. Rev. D 95, 056008 (2017).
[11] Y. Li, W. F. Wang, A. J. Ma, and Z. J. Xiao, Quasi-two-body decays $B_{(s)} \rightarrow K^{*}(892) h \rightarrow K \pi h$ in perturbative QCD approach, Eur. Phys. J. C 79, 37 (2019).

[12] A. J. Ma and W. F. Wang, Contributions of the kaon pair from $\rho(770)$ for the three-body decays $B \rightarrow D K \bar{K}$, Phys. Rev. D 103, 016002 (2021).

[13] Y. Y. Fan and W. F. Wang, Resonance contributions $\phi(1020,1680) \rightarrow K \bar{K}$ for the three-body decays $B \rightarrow K \bar{K} h$, Eur. Phys. J. C 80, 815 (2020).

[14] W. F. Wang, Will the subprocesses $\rho(770,1450)^{0} \rightarrow K^{+} K^{-}$ contribute large branching fractions for $B^{ \pm} \rightarrow \pi^{ \pm} K^{+} K^{-}$ decays?, Phys. Rev. D 101, 111901(R) (2020).

[15] N. Wang, Q. Chang, Y. L. Yang, and J. F. Sun, Study of the $B_{s} \rightarrow \phi f_{0}(980) \rightarrow \phi \pi^{+} \pi^{-}$decay with perturbative QCD approach, J. Phys. G 46, 095001 (2019).

[16] A. G. Grozin, On wave functions of meson pairs and meson resonances, Sov. J. Nucl. Phys. 38, 289 (1983).

[17] A. G. Grozin, One- and two-particle wave functions of multihadron systems, Theor. Math. Phys. 69, 1109 (1986).

[18] D. Müller, D. Robaschik, B. Geyer, F. M. Dittes, and J. Hořejši, Wave functions, evolution equations and evolution kernels from light ray operators of QCD, Fortsch. Phys. 42, 101 (1994).

[19] M. Diehl, T. Gousset, B. Pire, and O. Teryaev, Probing Partonic Structure in $\gamma^{*} \gamma \rightarrow \pi \pi$ near Threshold, Phys. Rev. Lett. 81, 1782 (1998).

[20] M. Diehl, T. Gousset, and B. Pire, Exclusive production of pion pairs in $\gamma^{*} \gamma$ collisions at large $Q^{2}$, Phys. Rev. D 62, 073014 (2000). 
[21] B. Pire and L. Szymanowski, Impact representation of generalized distribution amplitudes, Phys. Lett. B 556, 129 (2003).

[22] M. V. Polyakov, Hard exclusive electroproduction of two pions and their resonances, Nucl. Phys. B555, 231 (1999).

[23] Z. Rui, Y. Li, and H.n. Li, Four-body decays $B_{(s)} \rightarrow$ $(K \pi)_{S / P}(K \pi)_{S / P}$ in the perturbative QCD approach, J. High Energy Phys. 05 (2021) 082.

[24] V. L. Chernyak and A. R. Zhitnitsky, Asymptotic behavior of hadron form-factors in Quark model, Pis'ma Zh. Eksp. Teor. Fiz. 25, 544 (1977) [JETP Lett. 25, 510 (1977)].

[25] G. P. Lepage and S. J. Brodsky, Exclusive processes in quantum chromodynamics: Evolution equations for hadronic wave functions and the form-factors of mesons, Phys. Lett. 87B, 359 (1979).

[26] A. V. Efremov and A. V. Radyushkin, Factorization and asymptotical behavior of pion form-factor in QCD, Phys. Lett. 94B, 245 (1980).

[27] V. L. Chernyak, V. G. Serbo, and A. R. Zhitnitsky, Asymptotic hadronic form-factors in quantum chromodynamics, JETP Lett. 26, 594 (1977).

[28] C. Hambrock and A. Khodjamirian, Form factors in $\bar{B}^{0} \rightarrow$ $\pi \pi \ell \bar{\nu}_{\ell}$ from QCD light-cone sum rules, Nucl. Phys. B905, 373 (2016).

[29] J. P. Lees et al. (BABAR Collaboration), Precise measurement of the $e^{+} e^{-} \rightarrow \pi^{+} \pi^{-}(\gamma)$ cross section with the initialstate radiation method at $B A B A R$, Phys. Rev. D 86, 032013 (2012).

[30] W. F. Wang and H.n. Li, Quasi-two-body decays $B \rightarrow$ $K \rho \rightarrow K \pi \pi$ in perturbative QCD approach, Phys. Lett. B 763, 29 (2016).

[31] J. Hua, H. n. Li, C. D. Lu, W. Wang, and Z. P. Xing, Global analysis of hadronic two-body B decays in the perturbative QCD approach, Phys. Rev. D 104, 016025 (2021).

[32] H.n. Li and S. Mishima, Penguin-dominated $B \rightarrow P V$ decays in NLO perturbative QCD, Phys. Rev. D 74, 094020 (2006).

[33] Z. Rui, X. D. Gao, and C. D. Lü, Revisiting the $B \rightarrow \pi \rho, \pi \omega$ decays in the perturbative QCD approach beyond the leading order, Eur. Phys. J. C 72, 1923 (2012).

[34] H. n. Li, Y. L. Shen, and Y. M. Wang, Next-to-leading-order corrections to $B \rightarrow \pi$ form factors in $k_{T}$ factorization, Phys. Rev. D 85, 074004 (2012).

[35] R. Aaij et al. (LHCb Collaboration), Evidence for an $\eta_{c}(1 S) \pi^{-}$resonance in $B^{0} \rightarrow \eta_{c}(1 S) K^{+} \pi^{-}$decays, Eur. Phys. J. C 78, 1019 (2018).

[36] G. J. Gounaris and J. J. Sakurai, Finite-Width Corrections to the Vector-Meson-Dominance Prediction for $\rho \rightarrow e^{+} e^{-}$, Phys. Rev. Lett. 21, 244 (1968).

[37] P. A. Zyla et al. (Particle Data Group), Review of particle physics, Prog. Theor. Exp. Phys. 2020, 083 C01 (2020).

[38] Y. Y. Keum, H. n. Li, and A. I. Sanda, Penguin enhancement and $B \rightarrow K \pi$ decays in perturbative QCD, Phys. Rev. D 63, 054008 (2001).
[39] T. Kurimoto, H. n. Li, and A. I. Sanda, Leading-power contributions to $B \rightarrow \pi, \rho$ transition form factors, Phys. Rev. D 65, 014007 (2001).

[40] C. D. Lü and M. Z. Yang, $B$ to light meson transition form factors calculated in perturbative QCD approach, Eur. Phys. J. C 28, 515 (2003).

[41] H. n. Li, QCD aspects of exclusive B meson decays, Prog. Part. Nucl. Phys. 51, 85 (2003) and references therein.

[42] Z. J. Xiao, W. F. Wang, and Y. Y. Fan, Revisiting the pure annihilation decays $B_{s} \rightarrow \pi^{+} \pi^{-}$and $B^{0} \rightarrow K^{+} K^{-}$: The data and the pQCD predictions, Phys. Rev. D 85, 094003 (2012).

[43] A. G. Grozin and M. Neubert, Asymptotics of heavy-meson form factors, Phys. Rev. D 55, 272 (1997).

[44] Y. L. Yang, L. Lang, X. L. Zhao, J. S. Huang, and J.F. Sun, Reinvestigating the $B \rightarrow P P$ decays by including the contributions from $\phi_{B 2}$ Phys. Rev. D 103, 056006 (2021).

[45] V. M. Braun, Y. Ji, and A. N. Manashov, Higher-twist Bmeson distribution amplitudes in HQET, J. High Energy Phys. 05 (2017) 022.

[46] H. n. Li, Y.L. Shen, and Y. M. Wang, Resummation of rapidity logarithms in $B$ meson wave functions, J. High Energy Phys. 02 (2013) 008.

[47] Y. Y. Keum, H. n. Li, and A. I. Sanda, Fat penguins and imaginary penguins in perturbative QCD, Phys. Lett. B 504, 6 (2001).

[48] C. D. Lü, K. Ukai, and M. Z. Yang, Branching ratio and $C P$ violation of $B \rightarrow \pi \pi$ decays in perturbative QCD approach, Phys. Rev. D 63, 074009 (2001).

[49] A. Ali, G. Kramer, Y. Li, C. D. Lü, Y. L. Shen, W. Wang, and Y.M. Wang, Charmless nonleptonic $B_{s}$ decays to $P P, P V$ and $V V$ final states in the pQCD approach, Phys. Rev. D 76, 074018 (2007).

[50] K. M. Watson, The effect of final state interactions on reaction cross sections, Phys. Rev. 88, 1163 (1952).

[51] G. Breit and E. Wigner, Capture of slow neutrons, Phys. Rev. 49, 519 (1936).

[52] M. Tanabashi et al. (Particle Data Group), Review of particle physics, Phys. Rev. D 98, 030001 (2018) and 2019 update.

[53] P. Lepage and C. Gohlke, gplepage/lsqfit: lsqfit version 11.7, Zenodo. http://doi.org/10.5281/zenodo.4037174.

[54] H. n. Li, S. Mishima, and A. I. Sanda, Resolution to the $B \rightarrow \pi K$ puzzle, Phys. Rev. D 72, 114005 (2005).

[55] P. Ball, V. M. Braun, Y. Koike, and K. Tanaka, Higher twist distribution amplitudes of vector mesons in QCD: Formalism and twist three distributions, Nucl. Phys. B529, 323 (1998).

[56] Y. Li, Z. Rui, and Z. J. Xiao, $P$-wave contributions to $B_{(s)} \rightarrow$ $\psi K \pi$ decays in perturbative QCD approach, Chin. Phys. C 44, 073102 (2020).

[57] H. Y. Cheng, $C P$ violation in $B^{ \pm} \rightarrow \rho^{0} \pi^{ \pm}$and $B^{ \pm} \rightarrow \sigma \pi^{ \pm}$ decays, arXiv:2005.06080. 